alpine space - man \& environment vol. 13

Wolfgang Schöner, Silvia Prock, Birgit Sattler (Hrsg.)

\title{
Coole Forschung
} Lernen und Forschen im Internationalen Polarjahr 2007/2008 



\section{SERIES}

alpine space - man \& environment: vol. 13

Series Editors: Roland Psenner, Reinhard Lackner, Axel Borsdorf

Band 13

innsbruck university press 

Wolfgang Schöner, Silvia Prock, Birgit Sattler (Hrsg.)

\section{Coole Forschung}

Lernen und Forschen im Internationalen Polarjahr 2007/2008 


\section{Wolfgang Schöner}

Zentralanstalt für Meteorologie und Geodynamik (ZAMG), Wien

\section{Silvia Prock}

Büro für Öffentlichkeitsarbeit und Kulturservice, Universität Innsbruck

\section{Birgit Sattler}

Institut für Ökologie, Universität Innsbruck

Gedruckt mit Unterstützung des Bundesministeriums für Wissenschaft und Forschung - Sparkling Science, Bipolar sowie des Forschungsschwerpunkts Alpiner Raum - Mensch und Umwelt.

\section{BIP\$LAR}

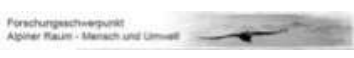

(C) innsbruck university press, 2012

Universität Innsbruck

Alle Rechte vorbehalten.

Das Werk ist urheberrechtlich geschützt. Die Rechte für Nachdruck, Übersetzung sowie der Entnahme von Abbildungen und Bildern liegen bei den Herausgeber bzw. bei den jeweiligen AutorInnen. Die in den Beiträgen geäußerten Meinungen müssen nicht mit denen der Herausgeber übereinstimmen.

Umschlag: Carmen Drolshagen

Umschlagmotiv: (C) Katarina Pavlovic, 2008

Layout: Reinhard Lackner

Produktion: Sterndruck GmbH, Fügen

www.uibk.ac.at/iup

www.uibk.ac.at/alpinerraum/publications/ 


\section{Inhalt}

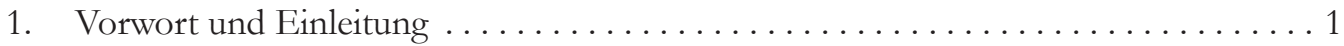

Vorwort BiPolar ...................................... 3

Marie Céline Loibl

Das Projekt BiPolar .................................. 5

Wolfgang Schöner

2. Brückenschlag Forschung - Bildung (Bridging Science and School) ........... 7

Sichtweisen der Wissenschaft .............................. 9

Birgit Sattler

Sichtweise „Hybrid“ (Lehrerin $=$ Wissenschaftlerin) $\ldots \ldots \ldots \ldots \ldots \ldots \ldots 11$

Michaela Panzenböck.

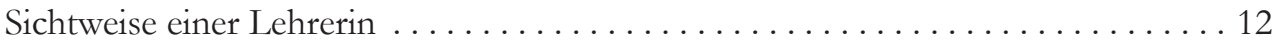

Silke Jung

3. Forschung ins Klassenzimmer (Science into School) $\ldots \ldots \ldots \ldots \ldots \ldots \ldots$

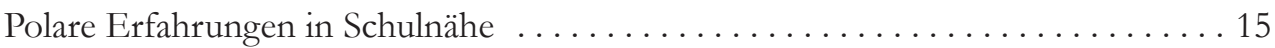

Franz Lechner, Sigrid Freinberger, Michaela Panzenböck

Das Klima im Baum - Schülerinnen betreiben Klimaforschung . . . . . . . . . . 18

Franz Lechner, Sigrid Freinberger, Michaela Panzenböck

Schülerexpedition im Extremlebensraum Hochgebirge .................. 21

Forschendes Lernen ist ein Instinkt $\ldots \ldots \ldots \ldots \ldots \ldots \ldots \ldots \ldots \ldots . \ldots . \ldots . \ldots . \ldots$

Markus Freiberger, Birgit Sattler

Von Schneealgen in der Arktis und in den Alpen, der „Kaiser-Max-Forelle“,

Bärtierchen in Drecklöchern am Gletschereis und Telefonaten mit

Forschern in der Antarktis . . . . . . . . . . . . . . . . . . . . . . . . 24

Markus Freiberger

Gletscherschwund und hartes Leben in der Kälte . ................... 27

Birgit Sattler

Gletscherwoche der 4 C Klasse (8.-12. September 2008)

Barbara Bauer-Moser 
Flechten - Bewohner extremer Lebensräume $\ldots \ldots \ldots \ldots \ldots \ldots \ldots \ldots \ldots . \ldots \ldots$

Waltrand Buchinger, Herwig Zeiler

Entwicklung und Selbstbau von einfachen Licht- und Temperaturmessgeräten . . 34

4. ForscherInnen der Zukunft (Scientists of the Future) .................. 39

Projektideen der SchülerInnen

Michaela Panzenböck ....................................... 41

Schlaflos in der Arktis? - eine Biorhythmusstudie $\ldots \ldots \ldots \ldots \ldots \ldots \ldots \ldots 4$

Michaela Panzenböck

Verrottungsversuch des BRG 6 Marchettigasse .................... 43

Bettina Girschick, Elfriede Schmid, Elisabeth Fuchs

KINKONG (der Kinderkongress in Wien) . . . . . . . . . . . . . . . . 44

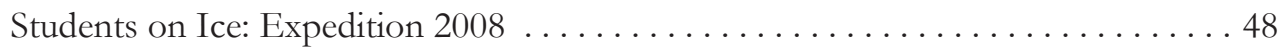

Michaela Lurger

Internationale Vernetzung - „Wir sind Expedition“ ................. 51

Birgit Sattler

Sparkling Science: From Lake Untersee, Antarctica, to the Icy Poles of Mars . . . . 53

Michael C. Storrie-Lombardi

Einbindung in andere internationale Programme $\ldots \ldots \ldots \ldots \ldots \ldots \ldots \ldots$

5. Wissenschaft geht an die Öffentlichkeit (Science goes public) … . . . . . . 59

Eisbär, Pinguin \& Co., Bettina Girschick, Elfriede Schmid ............... 61

Elisabeth Fuchs

Brettspiel BiPolar ....................................66 66

Silke Jung

Gemeinsame BiPolar - Ausstellung von der ErLEBnisschule

Mödling und dem BG Bachgasse, Mödling. .....................67 67

Herfried Weiss, Irene Wailzer

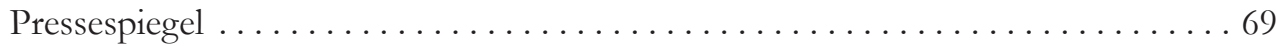

Silvia Prock 
6. Erfahrungen und Nachhaltigkeit, Evaluation $\ldots \ldots \ldots \ldots \ldots \ldots \ldots \ldots \ldots$

Erfahrung Wissenschaft ......................... 73

Birgit Sattler

Zwischenevalution

75

Silvia Prock

Bedingungen der Schulen für FBK-Projekte $\ldots \ldots \ldots \ldots \ldots \ldots \ldots \ldots \ldots \ldots \ldots \ldots \ldots$

Erfahrungen der SchülerInnen $\ldots \ldots \ldots \ldots \ldots \ldots \ldots \ldots \ldots \ldots \ldots \ldots \ldots \ldots \ldots \ldots \ldots \ldots \ldots$

Kooperation in BiPolar - Ein Blick von außen auf den

Brückenschlag zwischen Forschung und Schule .

Franz Radits, Anna Streissler, Franz Rauch 



\section{COOLE FORSCHUNG}

Lernen und Forschen im

Internationalen Polarjahr 2007/2008
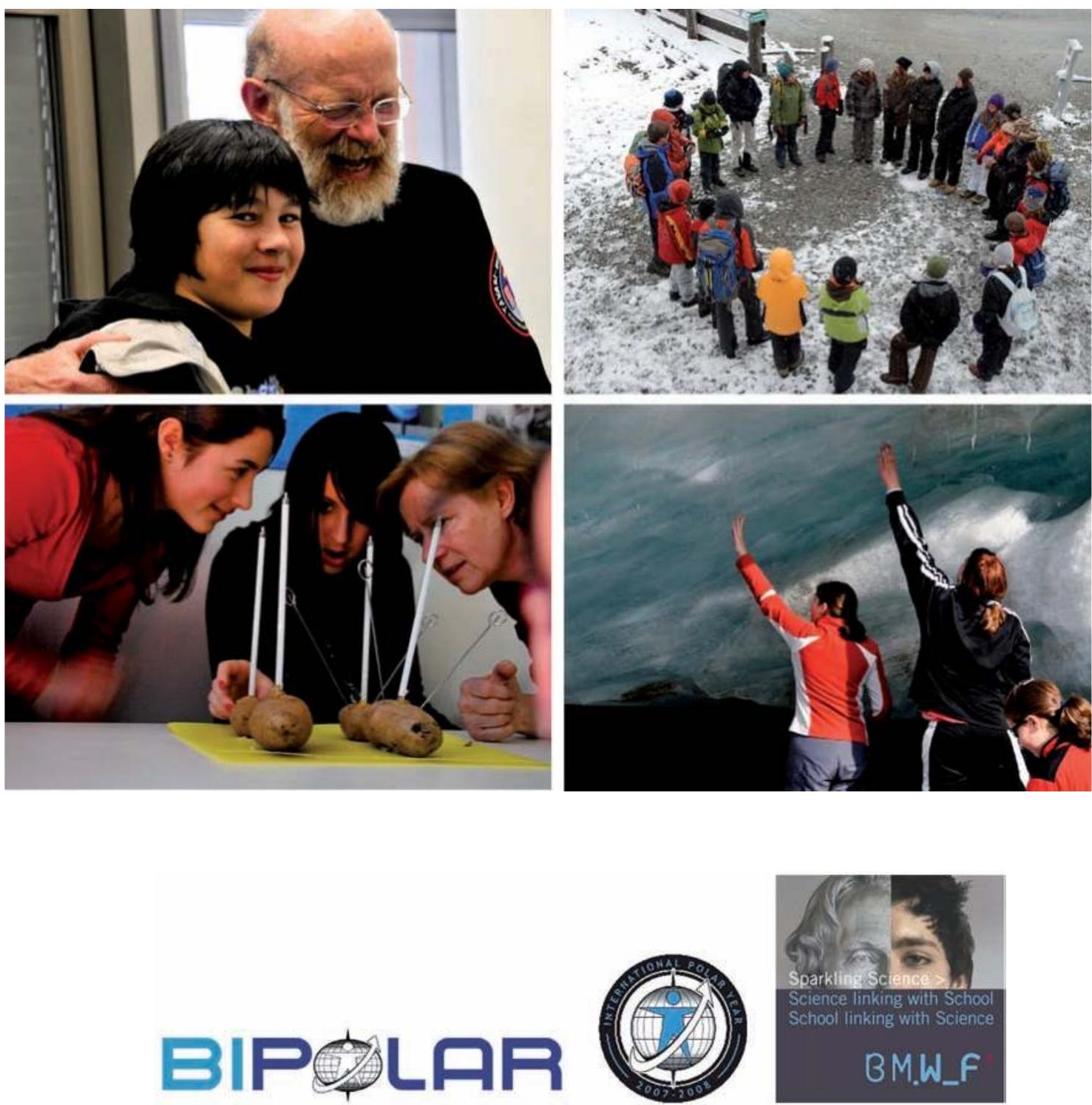



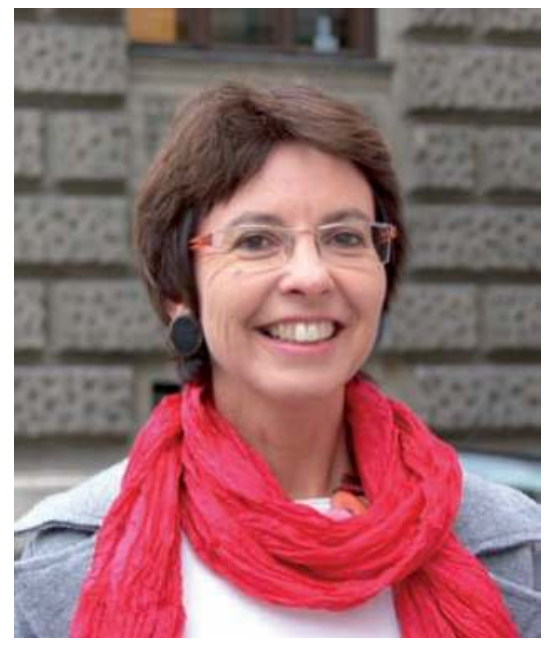

\title{
Vorwort BiPolar
}

\author{
Marie Céline Loibl, Bundesministerium für Wis- \\ senschaft und Forschung
}

Es gibt Projekte, die brauchen Mut.

Es gibt Projektteams, die bis an ibre Grenzen gehen und es gibt Projekte, die sich in völlig unerforschtes Gebiet vorwagen.

Das Vorhaben, über dessen Erfahrungen und Ergebnisse die vorliegende Publikation berichtet, erfüllte nicht nur eines, sondern alle drei dieser Kriterien, indem es sich auf ein schwieriges Experiment einließ, dessen Ausgang gänzlich ungewiss war.

Die beiden Ziele des Experimentes bestanden darin, Jugendliche so nahe an aktuelle Polarforschung heranzuführen, dass sie einen authentischen Einblick in laufende Forschungsarbeiten gewinnen würden und zu klären, ob Jugendliche bei intensiver Einbindung in wissenschaftliche Untersuchungen kleine Teilprojekte übernehmen und damit „echte“ Beiträge zu State of the Art Forschung leisten könnten.

Sich als international anerkannte Forscherin oder Forscher ernsthaft auf ein derartiges Experiment einzulassen, braucht Mut weil kaum jemand auf den ersten Blick das Gelingen eines solchen Versuches für möglich hält. An seine Grenzen stößt ein Projektteam, das so verschiedene Welten wie Schule und Wissenschaft vereint und dabei nicht nur die unterschiedlichen Spielregeln beider Welten einhalten muss, sondern auch neue Freiräume erschließen und dort gänzlich Ungewohntes ausprobieren will. Allen Beteiligten des vorgestellten Projektes - Schüler/innen, Wissenschaftler/innen und ganz besonders den Lehrer/ innen, die erfolgreich zwischen den beiden Welten vermittelt haben, gebührt für diese Leistung großer Respekt. Unerforschtes Neuland schließlich wurde in diesem Projekt mit der Erkundung von Forschungsansätzen und Methoden betreten, in welchen Schülerinnen und Schüler als Junior Scientists mitwirken und wissenschaftlich verwertbare Erhebungen und Auswertungen durchführen können.

Ein Glücksfall, wenn ein solcherart dreifach erfolgreiches Vorhaben als Pilotprojekt für ein Forschungsprogramm fungiert, mit dem das Bundesministerium für Wissenschaft und Forschung österreichweit Forschungsvorhaben fördern will, in welchen Jungendliche mitarbeiten können.

Das Projekt BiPolar, welches Sie auf den folgenden Seiten kennen lernen, ist ein solcher Glücksfall. Die erfolgreiche Durchführung des Projektes war ein entscheidender Faktor für die Realisierung des Forschungsprogramms Sparkling Science, das mit insgesamt 30 Mio Euro dotiert wurde und in dem seither weit über 700 Wissenschaftlerinnen verschiedenster Disziplinen gemeinsam mit tausenden Schüler/innen forschen und geforscht haben. 
Coole Forschung

Allen Mitgliedern des Projektteams darf ich im Namen des BMWF für diese wichtige Pionierarbeit und ihr großes Engagement danken.

Meine Gratulation zu den spannenden Ergebnissen und Alles Gute für viele weitere erfolgreiche Kooperationen!

Bundesministerium für Wissenschaft und Forschung Abteilung II/4: Forschung und Innovationen für die Zukunft Programmleitung: Dr. Marie Céline Loibl Teinfaltstraße 8 1014 Wien Tel: 01/53120-6926 Fax: 01/53120-99-6926

E-Mail: celine.loibl@bmwf.gv.at 


\section{Das Projekt BiPolar}

Wolfgang Schöner, Zentralanstalt für Meteorologie und Geodynamik

BiPolar war als ein Teilprojekt des Programms FERMAP des BMWF ein Pionierprojekt für Sparkling Science. Gleichzeitig war BiPolar auch ein Beitrag zum Internationalen Polarjahr 2007/2008 (www.ipy.org). Im Gegensatz zu den anderen Teilprojekten von FERMAP (Smaragd, Vegmon und Schools on Ice) beinhaltete BiPolar ausschließlich eine Forschungs-Bildungs-Kooperation, während der Forschungsteil unter dem Projektnamen FERMAP-Pilot durch die Österreichische Akademie der Wissenschaften finanziert wurde. Auf Grund der sehr starken finanziellen Kürzung von FERMAP-Pilot (Kürzung um 80\%), konnte das geplante Forschungsprogramm jedoch nicht beibehalten werden und musste auf ein Minimalprogramm reduziert werden.

In BiPolar wurde ein Modulsystem entwickelt, in dem am Beispiel der Polarforschung, die Einbindung von SchülerInnen in die Forschung und in die Entwicklung von Forschungsfragen erreicht werden sollte. BiPolar bestand aus 4 Modulen in denen schrittweise die Zusammenarbeit von Wissenschaft und Schule umgestzt wurde.

Die 4 Module des Projektes und ibre Inhalte:

MODUL1: Brückenschlag Wissenschaft - Schule

MODUL2: Wissenschaft in die Schule MODUL3: ForscherInnen der Zukunft MODUL4: Wissenschaft geht an die Öffentlichkeit

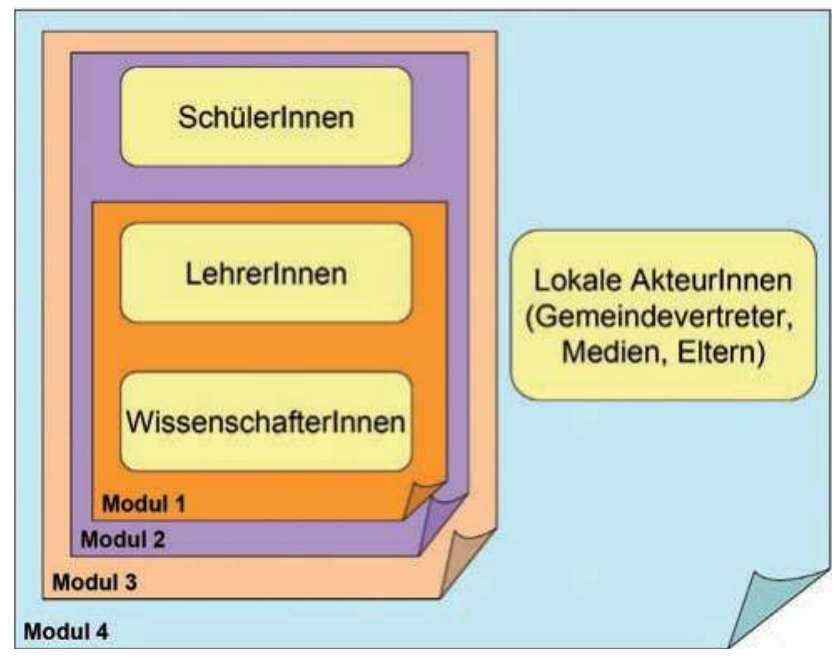

In dieser schrittweisen Vorgehensweise wurden die SchülerInnen an die Forschung herangeführt und entwickelten schließlich selbst eigene Forschungsfragen. Diese Forschungsfragen wurden in Form von kleinen Projekten durchgeführt und schließlich im Februar 2009 bei einem Kinderkongress an der Universität Wien vorgestellt. 
Wesentlicher Teil von BiPolar war auch eine Evaluation der Zusammenarbeit Forschung-Schule, sowohl intern als auch extern (durch das Austrian Educational Competence Center AECC der Universität Wien). Diese erfolgte sowohl zum Beginn sowie zum Ende des Projektes.

Im Bericht werden die Arbeiten zu den einzelnen Modulen detailliert dargestellt, wobei die Sichtweise von WissenschaftlerInnen, LehrerInnen und SchülerInnen Berücksichtigung findet. Die Arbeiten zu den vier Modulen konnten gemäß dem Projektantrag durchgeführt werden. Um Verwechslungen (BMVIT Programm „Forschung macht Schule") zu vermeiden, wurde der Titel der vorliegenden Publikation jedoch auf „Coole Forschung" abgeändert.

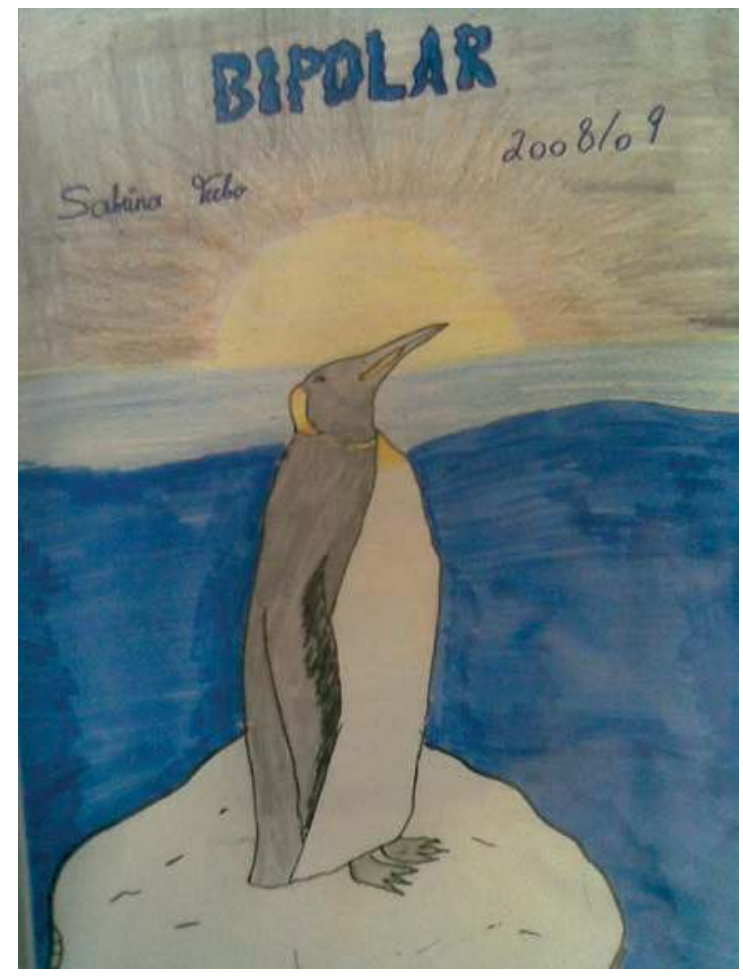

Bild von Sabrina Teebo, 2008. 


\section{Kapitel 2}

\section{Brückenschlag Forschung - Bildung (Bridging Science and School)}





\section{Brückenschlag Forschung - Bildung (Bridging Science and School)}

\subsection{Sichtweisen der Wissenschaft Birgit Sattler, Universität Innsbruck}

Wie schafft man es, LehrerInnen und WissenschafterInnen an einen Tisch zu bringen, um SchülerInnen aktuelle Forschung näher zu bringen und um sie selbst in die Forschung einzubinden?

Lange vor dieser Initiative gab es in Österreich das Vorhaben, eine so genannte „Science Week" zu veranstalten, in welcher die Universität erstmals auf die Straße gegangen ist, die Pforten für die Allgemeinheit geöffnet und die Wissenschaft transparent für Jedermann gemacht werden sollte. Ein nicht ganz einfaches Unterfangen, die Resonanz darauf ist trotz des innovativen und wichtigen Gedankens eher träge verlaufen. Offensichtlich existierte diese Hemmschwelle, von außen die Universität zu betreten, doch. Was zudem noch verwunderte, war auch die noch sehr zurückhaltende Reaktion von WissenschafterInnen, bei derartigen Aktionen mitzumachen, da es sich in diesem Zusammenhang um Wissenschaft 2. Klasse handeln würde. Doch zum Glück sind diese Ansätze nun beinahe 15 Jahre her und die Bereitschaft, Wissenschaft der Öffentlichkeit zu präsentieren, hat sich sehr gewandelt. Wenn man heute beobachtet, wie die Universitäten an Ereignissen wie dem „Aktionstag“ (eine Initiative der „Jungen Uni“, wo sich die Institute öffnen und Schulen einladen, zu ihnen zu kommen) gestürmt werden, so liegt der Gedanke nahe - der Brückenschlag ist geglückt!

Glücklicherweise finden sich auch LehrerInnen, die ebenso an einem derartigen Brückenschlag interessiert sind. Die Kooperation mit einer Forschungseinrichtung kann den Unterricht erleichtern oder vielfältiger gestalten, kann aber in gleicher Weise auch ein Quantum an Mehraufwand erfordern, zu dem eine Lehrperson erst einmal bereit sein muss.

Das wissenschaftliche Team zu BiPolar hat sich recht schnell zusammengefunden, nun galt es jedoch auch, die dafür bereiten LehrerInnen zu finden. Aussendungen an diverse Schultypen mit der schlichten Einladung, an diesem Projekt mitzuarbeiten, waren in den wenigsten Fällen von Erfolg gekrönt. Somit waren wir hauptsächlich auf Freunde bzw. bekannte Lehrpersonen beschränkt und das vollständige Team setzte sich aus diesem Kreis dann recht schnell zusammen. Die Chemie hat gepasst, dadurch wurden viele Hürden weitaus leichter genommen.

Im ersten Workshop zwischen LehrerInnen und WissenschafterInnen wurde erst einmal ein Sammelsurium von Ideen auf den Tisch gelegt: was ist machbar, was können wir bieten, welchen Aufwand bedeutet es für Schulen und Institute, welche Kooperationspartner kann man gewinnen. Für einen Wissenschaftler ist es eine große Herausforderung, mit SchülerInnen zusammenzuarbeiten und vor allem das gegenwärtige Niveau der SchülerInnen zu erkennen. Legt man die Latte zu tief, so fehlt der Anreiz, verschätzt man sich jedoch nach oben hin, so verliert man das Interesse der SchülerInnen. Aus dem gewohnten Milieu mit 
StudentInnen herauszutreten und das Forschungsgebiet nun Kindern zu erklären, ist oft schwieriger, als eine „herkömmliche“ Vorlesung zu halten. Es gelten für eine Zuhörerschaft im jungendlichen Alter schlichtweg andere Gesetze der Wertigkeit von Fakten. Wissen sollte „Cool“ sein, wenn man Geschichten dazu hört, umso besser - bitte nicht nur hard facts. Zudem sollte es „Action“ geben, die SchülerInnen wollen dabei etwas erleben. Es sollte einzigartig sein, sie von anderen Schülergruppen abheben können. Dies sind Anforderungen, mit welchen man im Universitätsalltag selten konfrontiert wird und die einen Wissenschafter ganz schön aus der Reserve locken können. Die Wissenschaft zur Bühne zu machen und das Wissen möglichst ,actionreich“ darzubieten, ist zudem ein Imagewandel, mit dem manche in der Forschung Tätigen sicher zu kämpfen haben.

Der große Vorteil des hier tätigen wissenschaftlichen Teams bestand darin, dass es sich durchgehend um Personen handelte, die hautnah mit diesem Thema beschäftigt sind, selbst auf Expedition gehen und daher selber auch die Auswirkungen des Klimawandels in Polargebieten erkennen. Aufgrund der in BiPolar gemachten Erfahrungen hat sich dieses Konzept bewährt, den SchülerInnen die Authentizität zu dem Thema zu geben und vor allem aus dem eigenen Erfahrungsschatz lehren zu können. Dies hat den Brückenschlag zu den SchülerInnen sehr erleichtert.

Ein weiterer großer Bonus waren die Ressourcen, welche für dieses Projekt genutzt werden konnten. Natürlich kann man keine Schulklasse in die Antarktis bringen, um einen Projektunterricht vor Ort zu veranstalten. Aber man kann sie in das ,kleine Polar“, dem Hochgebirge, mitnehmen. Was ökologisch auf den hohen Breitengraden passiert, kann man annäherungsweise auch auf Höhenstufen umlegen. Obwohl es für die SchülerInnen manchmal äußerste Überwindung war, dies zahlreichen Exkursionen auf Hochgebirgsstationen durchzuführen, so wurde das unmittelbare Erleben doch sehr geschätzt. Die umliegenden Stationen rund um den Sonnblick, den Gossenköllesee oder die Alpine Forschungsstelle in Obergurgl boten dafür unschätzbare Magnete und Authentizität für die SchülerInnen.

Ohne die Bereitschaft und die Flexibilität der LehrerInnen jedoch ist dieses Vorhaben unmöglich. Der Mehraufwand war in vielen Bereichen beachtlich, allein was den Organisationsaufwand für Lehrpersonen betrifft, wenn eine Schulklasse auswärts ist und die dabei verlorenen Stunden wieder aufgeholt werden müssen. In dieser Hinsicht sind die WissenschafterInnen glücklicherweise etwas freier in ihrer Zeiteinteilung.

Es mag auch schwierig sein, sich als WissenschafterInnen Schüler als Projektpartner vorstellen zu können. Partner, mit denen tatsächlich publizierbare Ergebnisse erzielt werden können. Doch im Rahmen dieses Konzeptes, in welchem SchülerInnen über eine lange Periode an ein Projekt gebunden sind, kann dies mit einer Kerngruppe auf jeden Fall funktionieren. Der Brückenschlag sollte durchaus nachhaltig sein, sofern es das Curriculum einer Schule auch zeitlich zulassen kann.

Aufgrund der Erfahrung mit der Hauptschule Zirl mögen die Kinder zwar wieder viel von dem Gelernten vergessen haben, aber sie sind mit diesem Thema mitgewachsen, haben es weit über die Grenzen hinaus auch mitgestaltet und ich würde mir sehr wünschen, dass sie im Zuge dieses Brückenschlages ein gestärktes Bewusstsein für Teamarbeit und diese Art von einzigartigen Ökosystemen gewonnen haben. 
Was bringt diese Zusammenarbeit einem/einer WissenschafterIn? Vielleicht wird diese Frage irgendwann obsolet sein und es wird zur Bringschuld, Wissenschaft der nächsten Generation weiterzugeben und sie mit ihnen zu erarbeiten. Wir geben ihnen Probleme in die Hand, welche vielleicht erst diese Generation lösen kann. Diese Kontinuität gelingt aber nur, wenn die notwendige Biegsamkeit in den respektiven Institutionen gewährleistet werden kann und wenn es gelingt, ,sich in der Mitte zu treffen“.

Die Arbeit mit Kindern bringt einem/einer WissenschafterIn neben den emotionalen Aspekten völlig neue Impulse, neue Perspektiven, andere Wertigkeiten der Fragestellungen. Aber wenn es gelingt, den Schülern Forschung aus unserer unmittelbaren Umgebung schmackhaft machen zu können, so wecken wir vielleicht auch eine Sensibilisierung dafür.

\title{
2.2. Sichtweise „Hybrid“ (Lehrerin = Wissenschaftlerin) Michaela Panzenböck, BRG Lilienfeld
}

\begin{abstract}
„Im Rabmen unserer Klimabündnismitgliedschaft sind Projekte, die sich mit klimarelevanten Themen beschäftigen, ein Schwerpunkt am BRG 6. Die Aktualität von BIPOLAR im Internationalen Polarjabr und vor allem der persönliche Kontakt mit Mag. Michaela Panzenböck waren die ausschlaggebenden Gründe für die Teilnabme am Projekt. Der Kontakt zur Wissenschaft war in diesem Projekt besonders reibungslos, da Mag. Panzenböck durch ibre Doppelrolle als Lehrerin und Wissenschaftlerin eine realistische Sicht auf die Umsetzbarkeit wissenschaftlicher Vorhaben ermöglichte. "
\end{abstract}

Forschungs- und Lehrtätigkeit an der Universität Wien, dazu seit 10 Jahren AHS-Lehrerin, diesen Spagat zu schaffen, dazu gehört schon eine gewisse Portion Schizophrenie. Aber genau diese Zweigleisigkeit war wichtig, um für BiPolar eine Art Brückenglied zwischen Wissenschaft und Schule darstellen zu können. Spannend für mich, erstmals meine beiden Tätigkeitsfelder verknüpfen zu können und in jedem Fall eine Herausforderung, ob ich mein Know-how jeweils einbringen kann. Bereits für die Projektkonzipierung war es wichtig, die in Schulen herrschenden Rahmenbedingungen zu kennen. Ebenso erleichterten persönliche Kontakte zu ehemaligen Studien- und jetzigen Lehrerkollegen die Kontaktaufnahme zu unseren Partnerschulen. In dem Zusammenhang war es für mich neben der Aufgabe, Begeisterung für die Polregionen zu vermitteln, ein besonderes Anliegen, LehrerInnen und SchülerInnen die oft bestehende Scheu vor der Wissenschaft oder den Wissenschaftern zu nehmen. Bei meiner Vortrags-Tournee durch unsere Partnerschulen in Niederösterreich und Wien wie auch bei der Einführung der SchülerInnen ins wissenschaftliche Arbeiten am Biozentrum der Universität Wien hoffte ich, diesbezüglichen Vorurteilen entgegenwirken zu können. Meinem Gefühl nach hat sich im Laufe unserer zweijährigen Projektlaufzeit eine sehr persönliche Ebene der Zusammenarbeit zwischen allen Beteiligten entwickelt, nicht zuletzt wegen der gemeinsam durchgeführten, außerschulischen Aktionen wie Gletscherexkursionen oder Kinderkongress. Das Zitat einer Schülerin „Wissenschafter lachen gerne“ sagt wohl einiges aus. 
Ein Vorteil für mich als sogenannter „Hybrid“ im Projektteam war sicherlich die pädagogische Erfahrung einerseits und der hautnahe Zugang zur Wissenschaft andererseits. Gewohnt, mit Kindern und Jugendlichen zu arbeiten, lässt man sich vom Verhalten einer Gruppe SchülerInnen nicht so leicht irritieren. Umgekehrt ist es immer einfacher, SchülerInnen für etwas zu faszinieren, das man selber erlebt hat. Einen weiteren Beitrag aus meiner „Hybridposition“ heraus konnte ich bei der Ideensammlung für die eigenständigen Forschungsprojekte liefern. Nicht nur die SchülerInnen, auch die LehrerInnen waren sichtlich überfordert mit der Aufgabe, Forschungsaufträge an die WissenschafterInnen zu übertragen, die dann vor Ort, in Grönland, durchgeführt werden sollten (s. Kap. 4). Unrealistische Vorstellungen zu methodisch einfachen und durchführbaren Versuchen umzulenken war nur Dank meiner Erfahrung als Forscherin möglich. Insgesamt waren jedoch die zwei Jahre Projektarbeit auch für mich sehr lehrreich; besonders beeindruckte mich das Engagement meiner Lehrerkollegen, ohne dem viele Veranstaltungen im Rahmen dieses Projekts nicht stattgefunden hätten.

\subsection{Sichtweise einer Lehrerin Silke Jung, BRG Sillgasse Innsbruck}

Der Kontakt zur Teilnahme an einem Sparkling Science Projekt anlässlich des Internationalen Polarjahres entstand durch das Institut für Ökologie der Universität Innsbruck. Als Absolventin unserer Schule offerierte Dr. Birgit Sattler uns die Möglichkeit, unser Wissen über die Arktis und Antarktis zu vertiefen. Für mich als Nicht-Biologin und Klassenvorstand bot sich die Chance fachübergreifend (Geschichte, Politische Bildung, Geografie, Biologie und Englisch) mit einem eLearning-Projekt daran teilzunehmen. Im Schuljahr 2008/09 begann dann meine Klasse an Teilbereichen zum Thema Arktis/Antarktis, wie Polartiere, Lebewesen und Nahrungsketten, Tourismus und Wirtschaft in den Polargebieten, Geschichte deren Erforschung, Bedeutung und Inhalt der Arktis- und Antarktisverträge, Unterschiede zwischen den Polgebieten und Lebensraum der Inuit, zu arbeiten. 


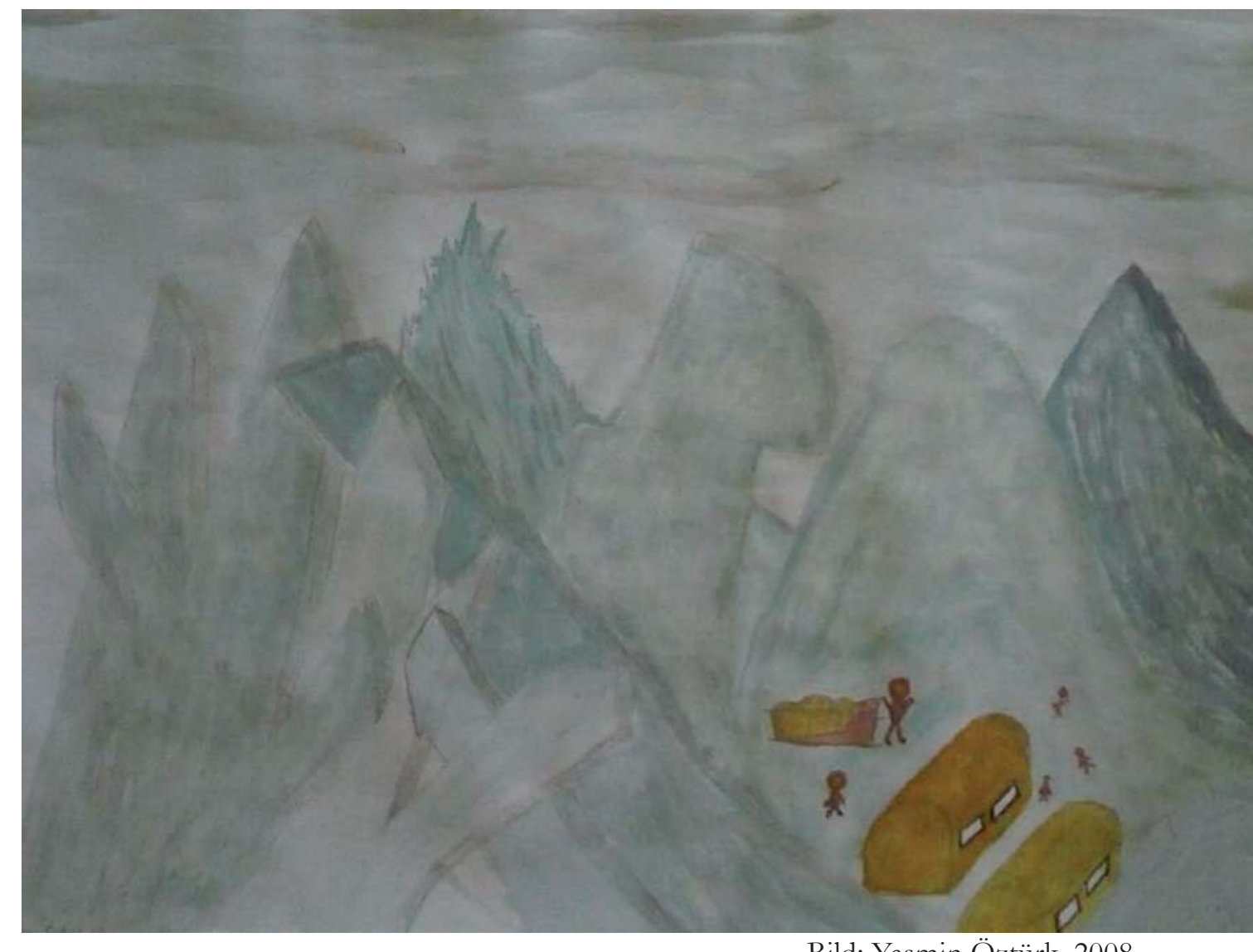

Bild: Yasmin Öztürk, 2008

\section{Kapitel 3}

Forschung ins Klassenzimmer (Science into School) 



\title{
3. "Bringing Science into the Classroom" (Forschung ins Klassenzimmer)
}

\author{
3.1. Polare Erfahrungen in Schulnähe \\ Franz Lechner, Sigrid Freinberger, Michaela Panzenböck \\ BRG Lilienfeld
}

Schneeuntersuchungen am Tirolerkogel:

Lilienfeld ist eine „Wiege des alpinen Schisports“ und so war es naheliegend, dass wir mit unseren SchülerInnen in unmittelbarer Schulnähe praktische Übungen zum Thema Schnee ausführen wollten. Die Schneearmut zu Winterbeginn zwang uns allerdings auf einen entfernteren Hausberg des Bezirks auszuweichen, nämlich auf den Tirolerkogel.

Ausgestattet mit Tourenschiern, Bords und Rodeln machten wir uns auf den Weg. Ein richtiger Wintertag erwartete uns mit ordentlicher Kälte, leichtem Schneefall und stürmischem Wind im Gipfelbereich. Die meisten SchülerInnen waren erstmals als „Tourengeher" im voralpinen Gelände unterwegs, trotzdem erreichten wir unseren wetterbedingten Versuchsstandort ohne allzu große Probleme.

Unter fachmännischer Anleitung unserer „Schneeexperten“ von der ZAMG und der örtlichen Polizei gruben wir einige Schneeprofile. Diese benutzten wir zur Analyse grundlegender Daten zur Schneephysik: Schichtung und Struktur des Schnees, Bewertung der Metamorphose, Messung der Temperatur und der Dichte. Nach kurzen theoretischen Einführungen durften wir selber Hand anlegen und sehr schnell konnten wir erfahren, dass scheinbar ganz einfache Methoden bei Kälte und Nässe eine ziemliche Herausforderung darstellten. Viele Schülerinnen beteiligten sich aktiv am Wiegen, Messen und Fotografieren, der Rest litt unter der Kälte.

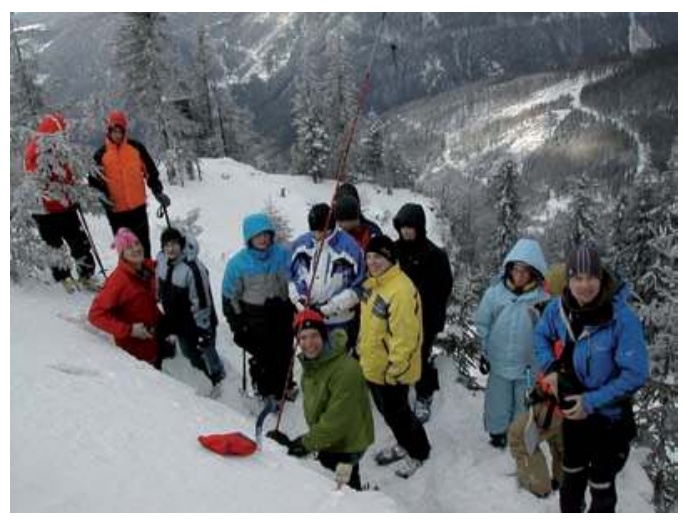

ScbülerInnen graben ein Schneeprofil. Foto: Franz Lechner

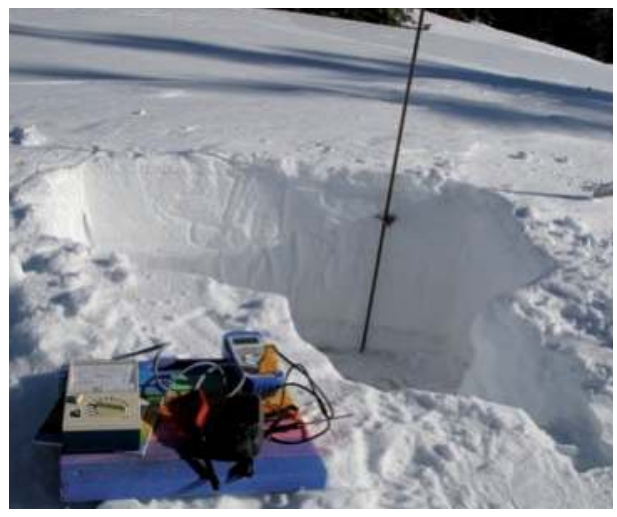

Ein Schneeschacht gibt Auskunft über Zusammensetzung und Schicbtung. Foto: Franz Lechner 


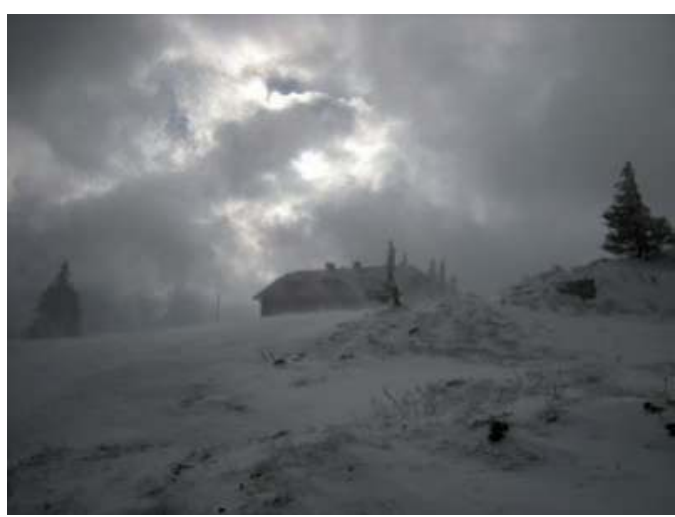

Die rettende Hütte am Tirolerkogel. Foto: Franz Lechner

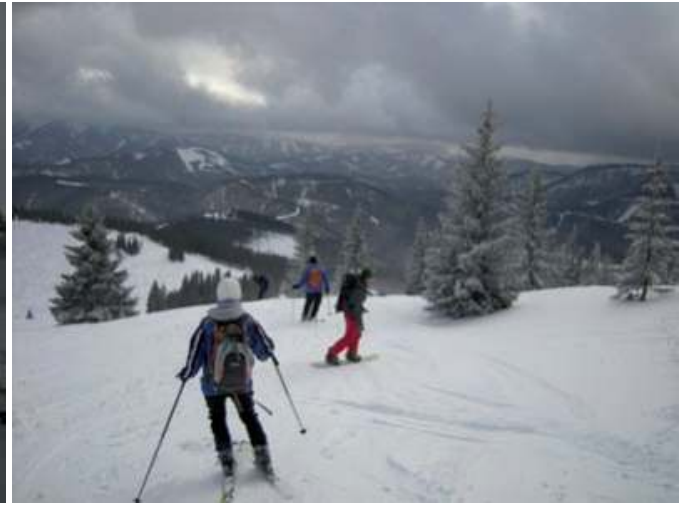

Abfahrt ins Tal nach getaner Arbeit. Foto: Franz Lechner

Alle Beteiligten konnten mittags in der warmen Hütte neue Kraft tanken.

Der Nachmittag gehörte den „Lawinenfachleuten“. Wieder wurden Schneeprofile gegraben und der Schnee in Hinblick auf seine Festigkeit untersucht. Ausgestattet mit Schaufel, Säge, Pinsel und Messinstrumenten konnten wir miterleben, wie Profis an die Beurteilung der Schnee- und Lawinensituation herangehen. Dazu kamen bewährte Methoden zur Überprüfung der Stabilität der Schneedecke zum Einsatz.

In der warmen Stube vermittelten die Herren vom Alpindienst der Polizei den Schülerinnen ihre Erfahrungen mit Tourengehern und bei Lawinenunfällen. So erhielten wir sehr viele praktische Tipps zur Verringerung des Risikos im freien alpinen Gelände. AbschlieBend präsentierte uns Herr Bieder sogar die Funktionsweise des Lawinenairbags.

Den „Schultag im Schnee“ krönte die Abfahrt zurück ins Tal. Für uns alle war dieser Teil sicher am spannendsten. Und unsere Schüler und Schülerinnen machten der „Wiege des Schilaufs" mit ihrem Können auf Brettln alle Ehre.

Der Winter 2008/09 sollte unser Wissen über den Schnee vertiefen. Wieder zwang uns der Schneemangel im Tal den Tirolerkogel als Versuchsstandort zu wählen. Auf einem kleinen Probeschneefeld im Gipfelbereich starteten wir im Jänner 2009 ein Monitoring des Schnees. In regelmäßigen Abständen haben wir die Probefläche besucht und unsere Messungen ausgeführt. Leider mussten wir sehr bald feststellen, dass die schulischen Messinstrumente insbesondere im Bereich der Schneechemie bei extremen Temperaturen unbrauchbar waren. So haben wir die Messungen abgebrochen und einzig die Veränderung der Schneestruktur bis zur Ausaperung im Mai verfolgt. 


\section{Datenanhang:}

Schultag im Schnee: 07.02.2008

7R, 8R und einzelne Schüler der 8GR (WPF BIUK)

Verantwortliche Lehrer:

Mag. Sigrid Freinberger, Mag. Franz Lechner, Mag. Michaela Panzenböck

ZAMG: Mag. Bernhard Hynek, Dr. Alexander Podesser

Leiter des Lawinenwarndienstes ZAMG Graz

Ersteller des Lawinenlageberichts

Polizei Lilienfeld - Alpindienst: Mjr. Michael Hochgerner, BezInsp. Hubert Bieder

Unterlagen zu den lokalen Wetterverhältnissen:

Herr Herbert Nutz, Liftgesellschaft Annaberg, ehemaliger Betriebsleiter

Seehöhe: Annaberg: 945m

Seehöhe Tirolerkogel: $1377 \mathrm{~m}$

Versuche zur Schneephysik und Schneechemie:

SchülerInnen der 7. und 8. Klassen erforschten einige physikalische und chemische Eigenschaften von Schnee auf experimentellem Wege. Natürlich kamen auch die theoretischen Hintergründe zur Sprache. Zu den Experimenten zählten Messungen der Schneedichte, der Leitfähigkeit von Schneeproben, des pH-Werts von Schnee, der Albedobestimmung (Rückstrahlung von Sonnenlicht), der Temperatur in der Schneedecke in verschiedenen Tiefen, der Wärmekapazität von Schnee und die Aufnahme der Schmelzkurve von Schnee. Einige der Messungen machten die SchülerInnen am Computer über ein angeschlossenes Interface (Ulab) und mittels verschiedener Sensoren. So konnten die Ergebnisse gleich am Monitor gezeigt werden.

Wir waren nicht nur erstaunt, wie nahe die von uns ermittelten Werte denen der Literatur kamen, unser so vertrautes Wasser/Eis hat uns auf energetischem Gebiet immer wieder überrascht. Die Gletscher wehren sich beharrlich gegenüber der flüssigen Phase. Unsere Messungen mit dem Kalorimeter haben das bestätigt. Diese Eigenschaft ist für die Existenz der Gletscher von höchster Bedeutung. Es sind enorme Energiemengen nötig, um Gletscher zu schmelzen.

Strategien und Anpassungen von Organismen bei Kälte und Frost:

Die in Polarregionen vorherrschenden niedrigen Temperaturen und deren Auswirkungen auf Organismen und Stoffkreisläufe waren auch Thema im Wahlpflichtfach Biologie der 7. Klassen. Strategien und Anpassungen von Organismen, um bei Kälte und Frost zu überleben, wurden ebenso untersucht wie die durch Kälte extrem verlangsamten Abbauprozesse in Tundraböden. In Experimenten wurden z.B. verschiedene Materialien wie Moschusochsenwolle auf ihre wärmeisolierenden Eigenschaften getestet oder gefrier- 
punkterniedrigenden Substanzen wie Glycerin getestet. Ein über Monate dauernder Versuch, bei dem die Temperaturverhältnisse in der Arktis simuliert wurden, demonstrierte, wie Kälte die Zersetzung organischer Substanzen hemmt. Ein weiteres Experiment ging der Frage nach, welchen Einfluss Temperatur und Nährstoffe auf bakterielles Wachstum haben. Die Auswertung dieses Versuchs - Färbung und Auszählung von Bakterien - fand am Department für Limnologie der Universität Wien statt.

\subsection{Das Klima im Baum - Schülerinnen betreiben Klima- forschung}

\section{Franz Lechner, Sigrid Freinberger, Michaela Panzenböck BRG Lilienfeld}

Im Rahmen des Projektes BiPolar habe ich mit einer kleinen Schülergruppe (WPF Geographie) versucht, einen eigenständigen Zugang zum Thema Klimawandel zu finden. Angeregt durch ein Buch der Zentralanstalt für Meteorologie1 versuchten wir über das Wachstum der Bäume Rückschlüsse auf das Klima zu finden.

Das Diagramm unten veranschaulicht, dass zur Rekonstruktion älterer Klimawerte auch die Dichte der Jahresringe verwendet werden kann.

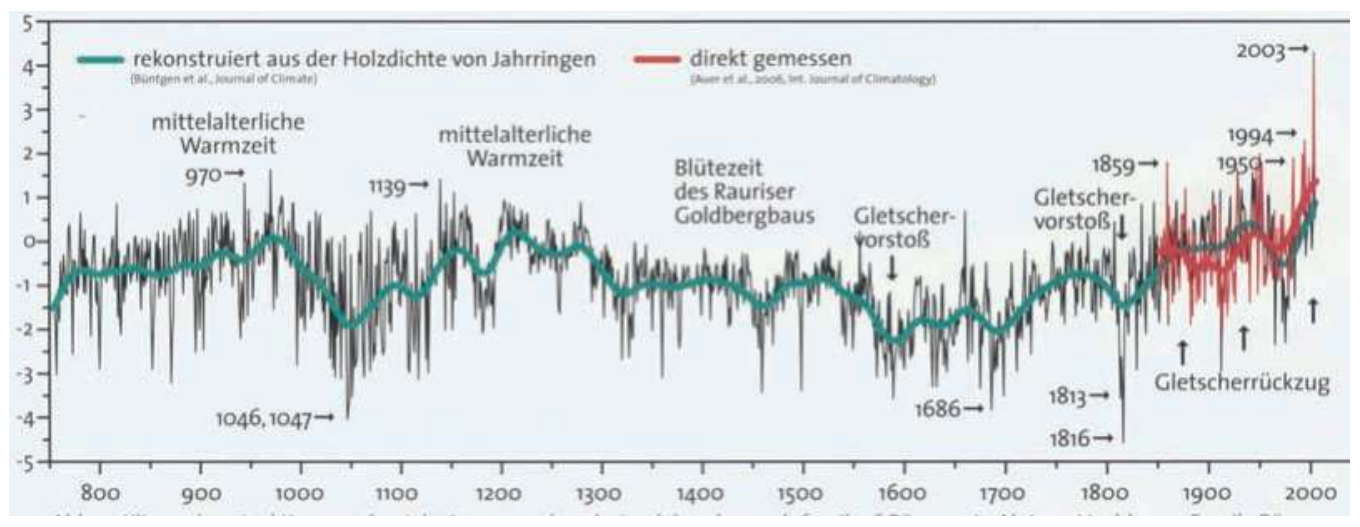

Abweichungen zum Mittel des 20. Jahrbunderts in Grad C. Klimarekonstruktion aus den Jabrringen von bunderten lebenden und ,fossilen "Bäumen in alpinen Hochlagen. Fossile Bäume aus früheren Zeiten findet man in Hochmooren, historischen Gebäuden, Alpenseen und im Schutt von Gletschermoränen. Quelle: U. Büntgen et al, 2006, R. Böbm et al, 2006. Abbildung aus ${ }^{1}$.

1 Gletscher im Klimawandel - Vom Eis der Polargebiete zum Goldbergkees in den Hohen Tauern, ZAMG Wien, 2007, Abb. 14 
Dipl. Ing. Dr. Michael Grabner vom Institut für Holzforschung an der BOKU Wien stellte mir Teile seiner Vorlesung zum Thema Dendrochronologie zur Verfügung, damit ich die Schülergruppe in die Probenbearbeitung und -interpretation einführen konnte. Er war sogar bereit nach Lilienfeld zu kommen, um bei der Auswertung unserer Proben mitzuhelfen.

Nach einer gründlichen theoretischen Einführung machte sich die Wahlpflichtfachgruppe an die praktische Arbeit. Das Ziehen der Proben konnte natürlich nur im Umfeld der Schule ausprobiert werden.

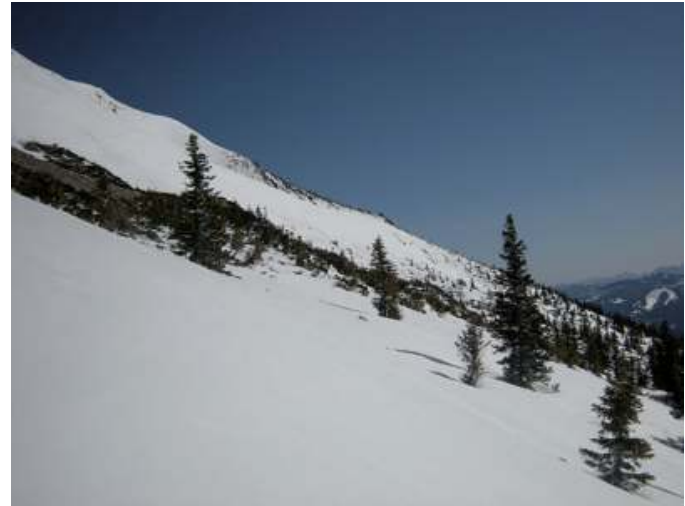

An der Waldgrenze ... Foto: Franz Lechner Sehr schnell erkannte die Schülergruppe, wie behutsam gearbeitet werden muss, damit eine brauchbare Probe gelingt. Die Proben wurden schließlich noch gesichert und nach dem Abtrocknen mit Schleifpapier perfekt ausgeschliffen.

Die Proben zur Klimabewertung stammten von der Südseite des Ötschers; die Forstverwaltung des Stiftes Lilienfeld ermöglichte die Beprobung einzelner Bäume an der Baumgrenze. Denn nur Bäume an Extremstandorten reagieren unmittelbar auf klimatische Veränderungen. Für die leichtere Bearbeitung wurden ausschließlich Fichten herangezogen, da das Auszählen der Jahresringe durch die deutliche Unterscheidung zwischen Früh- und Spätholz bei Fichten besonders einfach ist.

Als nächsten Arbeitsschritt benötigten wir klimatische Aufzeichnungen, welche längere Zeiträume darstellten und annähernd unserem Bearbeitungsgebiet standortmäßig entsprachen. Diese klimatischen Unterlagen erhielten wir für mehrere Stationen von der ZAMG Wien. Die Schülergruppe entschied sich für die Station Feuerkogel, weil diese Station nach unserer Einschätzung die höchste Übereinstimmung mit dem Ötscherstandort erbringen sollte. Somit war die nächste Aufgabenstellung, brauchbare Klimareihen mit dem PC anzufertigen; ein solches Ergebnis stellt das rechte Diagramm dar.

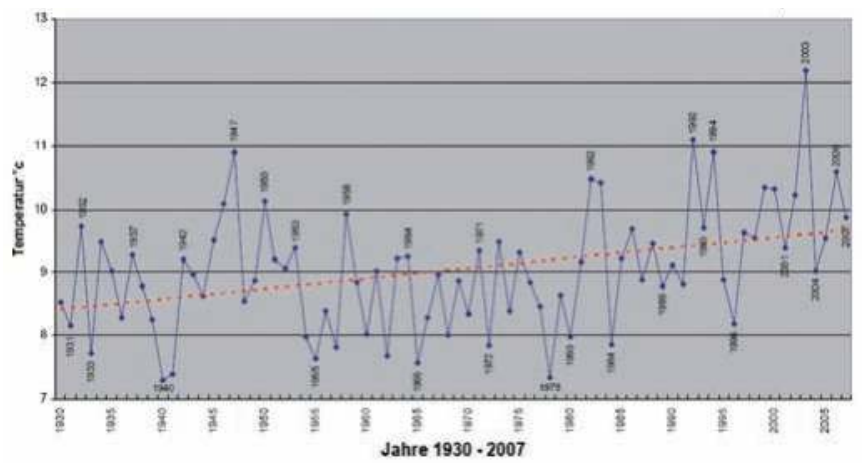

Sommertemperaturen Feuerkogel (Mai, Juni, Juli, August), 1618 m Seeböhe, Klimastation in den Nordalpen. Excel Bearbeitung durch die Schülergruppe 2008 


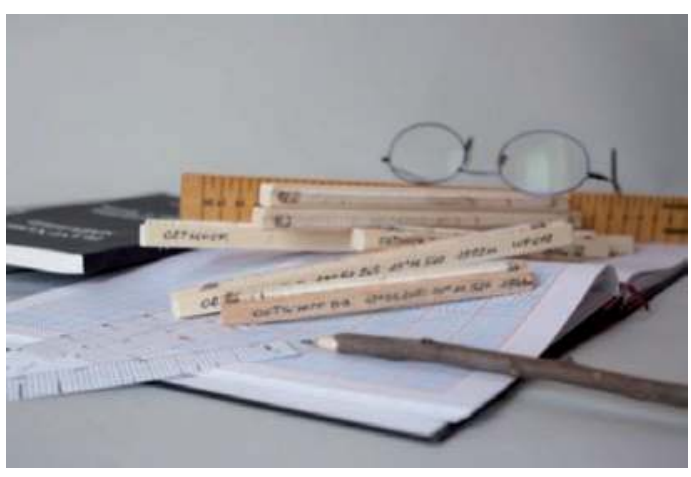

Stilleben vom Arbeitsplatz: Foto: Franz Lechner
Am spannendsten war schließlich die Auswertung unserer Proben. Vorerst mussten außergewöhnlich dünne und dicke Jahresringe auf Papierstreifen übertragen werden. Auch diese Arbeit verlangte viel Geduld und hohe Konzentration, damit brauchbare Ergebnisse erzielt werden konnten. Als letzten Schritt versuchten wir die Ergebnisse der einzelnen Bäume miteinander zu vergleichen. Die Resultate waren bei weitem nicht so signifikant, wie wir uns das erhofft hatten. Deutlich zu sehen war, dass klimatisch schlechte Jahre sich besser abbilden, als gute Jahre. So konnten die extrem warmen Sommer 1992, 1994 und 2003 nicht aus den Jahresringen abgelesen werden. Die kühlen Sommer der letzten Jahre waren ebenfalls an den Jahresringen nicht zu erkennen, während sich die extrem kalten Sommer 1941/42 in den Jahresringen widerspiegelten.

Die Aufgabenstellung der Dendroökologie hat für die Schülerinnen sehr aufschlussreiche Aspekte eigenständiger Forschungsarbeit zu Tage gefördert. Zum einen müssen viele Entscheidungen getroffen werden, wo gesichertes Wissen fehlt, zum anderen sind die Ergebnisse keinesfalls so eindeutig, dass aus wenigen Proben allgemein gültige Schlüsse gezogen werden können.

\section{Datenanhang}

Sommersemester 2008

Wahlpflichtfachgruppe Geographie und Wirtschaftskunde 7R und 7GR

Verantwortlicher Lehrer: Mag. Franz Lechner

Wissenschaftliche Betreuung: Dipl. Ing. Dr. Michael Grabner Institut für Holzforschung der BOKU Wien

Klimatische Unterlagen: $\quad$ ZAMG Wien, Mag. Bernhard Hynek

Praktische Hilfestellungen: Forstverwaltung des Stiftes Lilienfeld Oberforstmeister DI Franz Scheibenreif DI Klaus Kratzer 


\subsection{Schülerexpedition im Extremlebensraum Hochgebirge}

Aus dem Tagebuch einer elfjährigen Teilnehmerin der HS Zirl der 1. Tiroler hochalpinen Schülerexpedition

\section{Jänner 2008}

Birgit Sattler, eine Polarforscherin, besucht uns am 24. Jänner 2008 in der Hauptschule Zirl. Sie erzählt uns über Polarforscher, Entdecker und Pioniere, erklärt uns, dass es Leben im Eis gibt und zeigt Bilder vom Alltag der Forscher, die heutzutage in der Arktis und Antarktis forschen. Unsere Klasse soll bei einem größeren Projekt teilnehmen.

\section{Feber 2008}

Unsere beiden Klassenvorstände Markus Freiberger und Andrea Hoffmann waren in Wien auf der ZAMG. Sie erklären uns, dass wir von der UNI Innsbruck Forschungsaufträge bekommen. Wir sollen in den nächsten Wochen vier Gruppen bilden. Limnologen, Chemiker, Zoologen und Botaniker. Unsere Lehrer erklären uns: „Ihr sollt nachempfinden, wie es Polarforschern bei ihrer Arbeit ergeht. Die Bedingungen im Hochgebirge ähneln jenen in den Polargebieten. Dort findet man Leben an der Grenze. Was liegt also näher, als mit euch ins Hochgebirge zu gehen; und zwar, wenn dort noch Winter herrscht; aber nicht mit Liften als Aufstiegshilfe, sondern mit Schneeschuhen. Am Berg soll dann geforscht werden. Als Forschungsstätte bietet sich eine Station des Limnologischen Institutes der UNI Innsbruck an, sie steht im Kühtai am Gossenköllesee in 2400 m Höhe.“

\section{Expeditionsvorbereitung}

Vier Buben werden Chemiker, sie analysieren Wasser. Ich bin mit drei Mädchen in einer Gruppe, die Mikroskopieren lernt. Eh ganz interessant. Dann kommt von unseren Klassenvorständen eine Ankündigung: Wir müssen am Wintersporttag als Vorbereitung für die Expedition neben der Piste zur Skihütte am Gschwandtkopf marschieren - mit Gepäck. $\mathrm{Na}$ ja, Vergnügen ist das keines!

\section{Feber 2008: Training mit Schneeschuhen}

Es hat in der Nacht geschneit. Unsere Eltern bringen uns nach Seefeld. $20 \mathrm{~cm}$ Neuschnee - Pulver. Nach einer halben Stunde sind auch die patscherten Buben fertig. 9.30, der Nebel verzieht sich, die Sonne kommt heraus. Die 3a fährt gerade durch den Pulverschnee und legt die ersten Spuren. Wahnsinn - und wir stapfen durch den Tiefschnee. Nach zwei Stunden sind wir am Ziel. Alle haben es geschafft. 


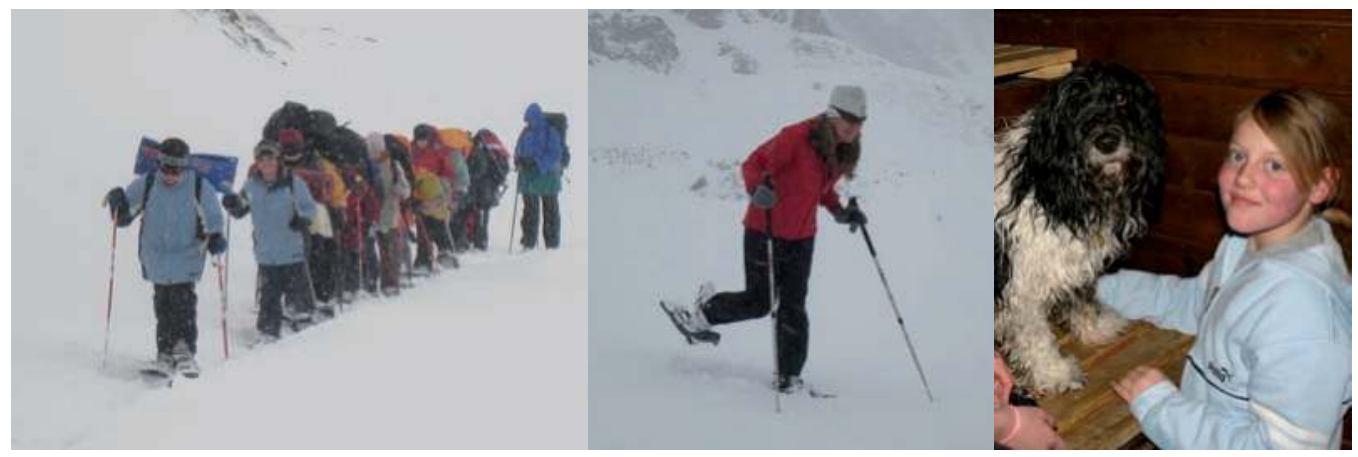

Impressionen von der Schneeschubwanderung. Fotos: Silvia Prock

\section{April 2008 - Der Expeditionstag}

Der Wetterbericht meldet um $7.00 \mathrm{Uhr}$ : Temperatursturz, $-2^{\circ}$ in $2000 \mathrm{~m}$, vormittags noch Schneefall dann leichte Wetterbesserung. So hautnah wollten wir das eigentlich nicht erfahren, wie es Forschern in den Polargebieten geht. Kein Mensch ist hier. Die Lifte stehen still, keine Pistenraupe rattert über die Hänge, in den Hotels brennt kein Licht. Dafür $20 \mathrm{~cm}$ Neuschnee - schon wieder. Wir legen die Schneeschuhe an, stapfen im Gänsemarsch Frau Silvia Prock von der Jungen Uni nach. Mit uns marschiert der Hund unseres Lehrers - „Ein echter Polarhund“, hat er gesagt. Na ja, sieht nicht ganz so aus. Man erkennt keinen Weg. Nebel steigt auf und es schneit immer stärker. Noch zwei Stunden bis zur Hütte. Endlich sehen wir wieder die Liftstützen. „Wir sind am richtigen Weg“, sagt man uns. Wir keuchen hinterher. Dann nach zwei Stunden sehe ich einen roten Anorak. Birgit Sattler, die Polarforscherin, springt uns entgegen: „Nur noch zehn Minuten bis zur Hütte!“ Geschafft! Wir bekommen heißen Tee und Frankfurter. Ich hab mich gleich wieder erholt. Denn schon geht es los mit den Forschungsaufträgen. Sie werden uns vorgelesen und dann wieder hinaus ins Freie. Es hat Gott sei Dank aufgehört zu schneien und die Sonne scheint sogar durch die Wolken. Wir stapfen auf den See und bekommen einen Eisbohrer zugereicht. Die erste Überraschung: Kein Wasser nach einem Meter. Also Verlängerungsgestänge aufgesteckt. Nach eineinhalb Meter wird es mühsam. Später werden wir wissen warum. Die letzte Schicht ist glasklares, massives Eis. Black Ice nennen es die WissenschafterInnen. Zwei Meter, der Bohrer bricht durchs Eis, endlich, mir tun schon die Arme weh. Vier andere Schüler vermessen den Kern, zeichnen ihn ab und Birgit Sattler erklärt, warum alles so geschichtet ist. Meine Gruppe nimmt eine Wasserprobe. Im Labor fertigen wir ein mikroskopisches Präparat an. Wir finden kleine Lebewesen. Auch die zeichnen wir ab. Mit den Ergebnissen sollen wir in den nächsten Wochen auf die Uni. Silvia Prock, die Botanikerin, kratzt mit ein paar Buben etwas Buntes von einem Stein. Die kommen sicher nicht drauf, was das ist. 


\section{Die Begleiter}

Auf der Station hatte ein ORF Team auf uns gewartet. Sie filmen alles und interviewen uns. Wir sind ziemlich aufgeregt, freuen uns aber auf die Gaudi in der Nacht. Wir übernachten alle auf Matten und in Schlafsäcken am Boden in der Station. Wie die Studenten, sagt man uns. Morgen geht's wieder ins Tal.

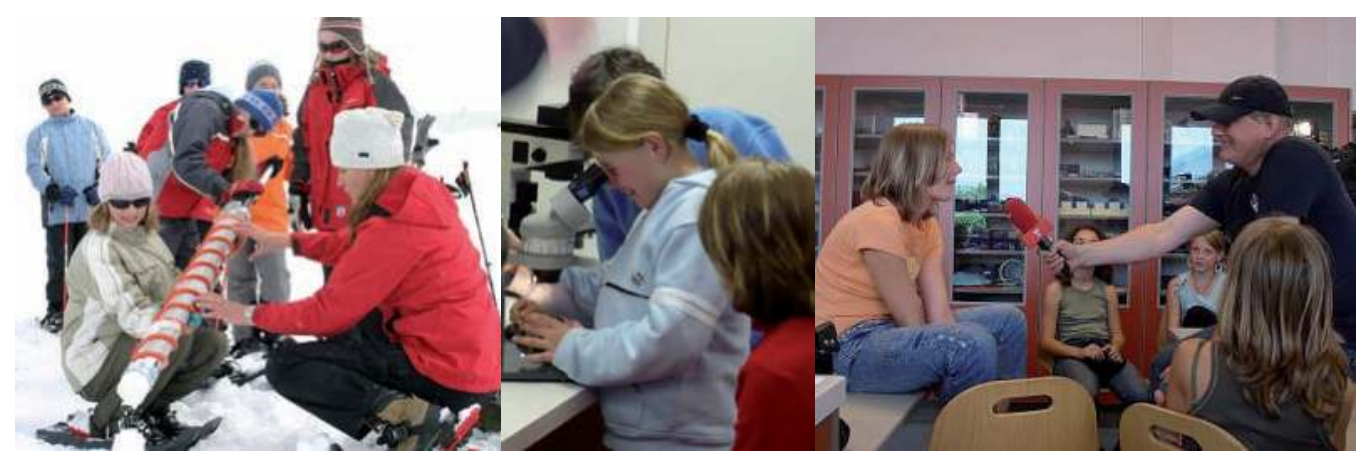

Jungforscher bei der Arbeit. Fotos: Silvia Prock, Markus Freiberger, Birgit Sattler

\section{Mai 2008 - Wieder in der Schule}

Wir sind unheimlich stolz. Wir haben es geschafft und durften zwei Tage Forscher sein. Ein Bericht von uns kam in „Tirol heute“ und von Wien erhielten unsere Lehrer ein E-Mail, dass unser Projekt einen Preis gewonnen hat. Jetzt geht's nach Wien. Davor aber noch auf die UNI Innsbruck - Forschungsergebnisse abliefern. Bin gespannt, was wir entdeckt haben.

\subsection{Forschendes Lernen ist ein Instinkt Markus Freiberger, HS Zirl Birgit Sattler, Universität Innsbruck}

Man muss sich nur selber zurückerinnern: Unter welchen Voraussetzungen haben wir am meisten gelernt? Wenn uns jemand etwas vorgelesen hat, oder wenn wir selber als Kinder etwas an- und somit begreifen konnten?

Forschendes Lernen ist im Grunde kein neues Konzept, sondern vielmehr ein Instinkt. Die Realisierung der in BiPolar gestellten Forschungsfragen konnte aus der Warte des Projektteams nur anhand von „hands-on“ Methoden bearbeitet werden. Wir können den SchülerInnen von unserer Forschung erzählen, ihnen Bilder davon zeigen, aber es geht wohl nichts über den direkten Bezug zum Forschungsobjekt. Dazu muss man den SchülerInnen die Wissenschaft direkt zu ihnen bringen.

Wie bereits eingangs erwähnt, verfügen die teilnehmenden Institute über einmalige Ressourcen, was Forschungsanlagen vor Ort betrifft. Die WissenschafterInnen sind allesamt in aktuellen Projekten in Polargebieten und im Hochgebirge beschäftigt, so konnte 
den SchülerInnen nun auch die Möglichkeit gegeben werden, nicht nur über Forschungsergebnisse zu erfahren, sondern sie auch selbst zu erheben. Ein kleines Abenteuer, wenn man bedenkt, dass man sich mit einer Horde von 11-12 Jährigen immer wieder im Hochgebirge befindet, aber auf diese Art wurde das Freiland zum erweiterten Klassenzimmer gemacht.

\subsection{Von Schneealgen in der Arktis und in den Alpen, der „Kaiser- Max-Forelle", Bärtierchen in Drecklöchern am Gletschereis und Telefonaten mit Forschern in der Antarktis}

\section{Markus Freiberger, HS Zirl}

\section{Roter Schnee}

16. August $1818,75^{\circ}$ nördliche Breite, zwischen Eisbergen segelt das Forschungsschiff „Isabella“ nordwärts. Konteradmiral John Ross und seine Besatzung sind auf der Suche nach der Nordwestpassage. Nach wochenlanger Fahrt durch die arktische Welt aus eintönigem Weiß und Grau machen sie an den steil aufragenden Felsufern der Baffins Bay eine verwirrende Entdeckung, ein ausgedehntes Schneefeld von leuchtend roter Farbe. Die Seeleute haben keine Ahnung, warum dieser Schnee rot war. Sie mutmaßen es sei Blut von einem Tier, Meteoritenstaub oder Vogeldreck. Der Kapitän machte einen Blick durchs Bordmikroskop und schrieb in seinem nach der Rückkehr 1819 veröffentlichten Tagebuch: „Die Substanz scheint aus gleich großen Teilchen in der Gestalt von kleinen Samenkörnchen von dunkelroter Farbe zu bestehen." Später vermuteten einige Wissenschaftler des 19. Jahrhunderts es sei ein Pilz, was da entdeckt wurde. Bis man erkannte, dass es sich um einzellige Algen, um Schneealgen handelt, sollten noch Jahrzehnte vergehen.

Ein Schauplatzwechsel: Frühjahr 2009: Silvia Prock unternimmt eine Bergwanderung im Ötztal. Am Fuße des Achertkogel, in 2000m Seehöhe entdeckt sie ein Schneefeld, das rot gefärbt ist. Schneealgen. Man findet sie hier genauso wie in den Polarregionen und kaum ein Wanderer oder Schitourengänger weiß, was der rote Schnee zu bedeuten hat. Am Lehrplan der Sekundarstufe I wird im Fach Biologie und Umweltkunde in Österreich zwar ausführlichst (in der 5. und 7. Schulstufe) auf jene Tiere und Feldfrüchte eingegangen, die unsere Landwirte produzieren, vom roten Schnee erfahren die jungen Alpenbewohner aber nichts.

Außer der Wetterbericht meldet wieder einmal Wüstensand, den es von der Sahara über die Gipfel weht, aber dann wird der Schnee leicht orange, nie so intensiv rot.

Was werden also Kinder über diesen Schnee denken? Die Botanikerin Silvia Prock nimmt Proben mit, behelfsmäßig in einer leeren Getränkeflasche, weil sie eigentlich nur wandern und nicht für SchülerInnen Forschungsmaterial sammeln wollte. Die Proben werden abends in die Hauptschule Zirl gebracht und warten im Kühlschrank des Physiksaals auf ihr weiteres Schicksal. Am darauf folgenden Tag stehen sie vor 10 Schülern der vierten Klasse. Der Schnee ist inzwischen geschmolzen, im Eiswasser schwimmen rote Klumpen. Die Schüler, welche das Schwerpunktfach Ökologie gewählt haben, erhalten den Auftrag, die Substanz zu untersuchen. Ihnen wird ein Foto von der Stelle gezeigt, wo die Proben genommen wurden. Ein rotes Schneefeld. Das Verblüffende: Die 13-14 Jahre alten SchülerInnen stellen ähnliche Hypothesen auf, wie die Männer am Schiff der Isabelle vor fast 200 
Jahren: Die Jugendlichen meinen, es sei Saharasand, Blut, Staub von Meteoriten, und - was es damals noch nicht gab: Farbe von der Pistenmarkierung.

Auch die Untersuchung mit den Stereolupen brachte keine neuen Erkenntnisse, aber zumindest ein paar saubere Zeichnungen. Im 21. Jahrhundert haben es „Forscher“ leichter, man kann googeln. Zu welchen Ergebnissen die SchülerInnen auf diese Weise kamen, möchte ich am Schluss dieses Berichtes erläutern.

\section{Von Jungforschern und Umweltdetektiven, die interdisziplinär arbeiten}

Drehen wir das Rad der Zeit um noch ein Jahr zurück. Im Frühjahr 2008 kam es zum ersten Treffen von Wissenschaftlern und LehrerInnen auf der ZAMG in Wien anlässlich des Projektes BiPolar. Unter dem Schlagwort „ForschungMachtSchule“ werden die ersten Ideen ausgetauscht, wie man Schülern Wissenschaft und Forschung näher bringen kann, im Speziellen anlässlich des Polarjahres 2007/08. Für zwei Tiroler Lehrer und 16 SchülerInnen, die das Schwerpunktfach Ökologie in der Hauptschule Zirl gewählt haben die einmalige Chance mit einer Polarforscherin, Dr. Birgit Sattler vom Institut für Ökologie der Uni Innsbruck zusammenzuarbeiten.

Wie könnte man also Kinder das Arbeiten von WissenschaftlerInnen am besten nachvollziehen, es vielleicht sogar miterleben lassen? Birgit Sattler war schon im Herbst zu einem Vortrag in die Schule gekommen, zeigte Bilder von ihren Expeditionen in den arktischen Regionen, was die Zehnjährigen stark beeindruckte. Wer wollte nicht in diesem Alter Astronaut, Pilot oder Wissenschaftler werden? „Machen wir eine Schülerexpedition!“ Diese Idee hatte als erste „unsere“ Wissenschaftlerin Birgit Sattler. Folgendes Konzept wurde daraufhin von dem kleinen Team bestehend aus der Geographielehrerin Andrea Hoffmann, mir, der ich seit zwanzig Jahren Biologie und Ökologie an verschiedenen Hauptschulen unterrichte, Dr. Silvia Prock von der Jungen Uni und Dr. Birgit Sattler, Mikrobiologin und Limnologin, spezialisiert auf Leben am Eis erarbeitet. Die SchülerInnen sollen Arbeitsaufträge von Forschern und Wissenschaftlern der UNI Innsbruck bekommen, von verschiedenen Disziplinen. Dadurch sollten sie auch jenen Aspekt der heutigen Wissenschaft miterleben können, ohne die Forschung nicht mehr denkbar ist. Wissenschaftler arbeiten heute anders als vor 200 Jahren interdisziplinär. So wurden aus den 10-Jährigen: Botaniker, Limnologen, Zoologen und Chemiker, wobei letztere sich mit ihrem Gewässer-Prüfkoffer mehr als Umweltdetektive fühlten. Was sie bei ihrer ersten hochalpinen Schülerexpedition auf die Limnologische Forschungsstation am Gossenköllesee im Kühtai erlebten, kann man im Kapitel 3.3 nachlesen. Neben den Erkenntnissen, die sie bei ihren Forschungen gewannen, blieben auch zwei kleine Enttäuschungen. Die Jungbotaniker, die von Dr. Silvia Prock (einer echten Botanikerin) betreut wurden, sammelten Proben von Flechten, bunten Steine - wie sie im Forschungsauftrag genannt wurden und untersuchten sie unter dem Mikroskop. Geschult durch einen Mikroskopierkurs, bei dem Algen aus dem Schulaquarium und ein Champignon Gegenstände der Untersuchung waren, erkannten die vier Mädchen, dass auf dem Objektträger sich wahrscheinlich Algen befanden und eine Schülerin erkannte auch Pilzfäden. Dass hier aber ein Zusammenhang bestehen könnte, diese Mutmaßung wurde von keinem Kind angestellt. Der Begriff Symbiose wird auch erstmals in der 6. Schulstufe 
erwähnt und ohne dieses Wissen muss man als Jungforscher zwangsläufig „scheitern“. Den Zoologen ging es noch schlimmer, es war ihnen nicht gelungen, eine jener seltenen „KaiserMax-Forellen", deren Population seit über 500 Jahren in dem Bergsee lebt, der Ziel der Expedition war, zu fangen und zu untersuchen. Die Gruppe musste also noch einmal hinauf

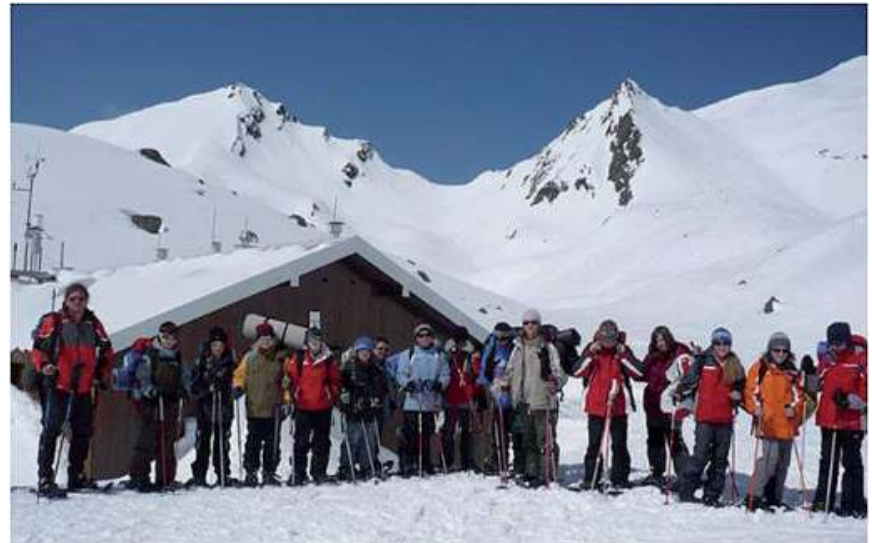

Die Forschergruppe vor der limnologischen Station am Gossenköllesee (Kübtai, Tirol). Foto: Markus Freiberger auf $2400 \mathrm{~m}$, neues Ziel war den Lebensraum über einen längeren Zeitraum zu beobachten. Damit war das „Schülermonitoring“" erfunden.

Im Juni des gleichen Jahres marschierten wir mit der Gruppe ein zweites Mal zum Gossenköllesee, wo sich die limnologische Station der UNI Innsbruck hoch über dem Kühtai befindet. Diesmal konnte ich als alter Fischer etwas beitragen. Ich hatte einen "fishfinder", ein Echolot mitgenommen. Mit dem Boot fuhr die erste Klein-

gruppe hinaus. Das Gerät zeigte am kleinen Display die Wassertiefe an, aber keine Signale von Fischen, bis wir die Seemitte erreichten, 9m Tiefe und plötzlich die ersten Signale. Immer wieder konnte man erkennen, dass Fische knapp über dem Grund vorbeizogen. An der Angelrute war als Köder eine so genannte Hegene montiert. Mit diesem Kunstköder fängt man in den tiefen Alpenseen Renken. Der Kunstköder imitiert Larven von Steinfliegen, Eintagsfliegen u.ä.. Selbstverständlich waren die Widerhaken zur Schonung

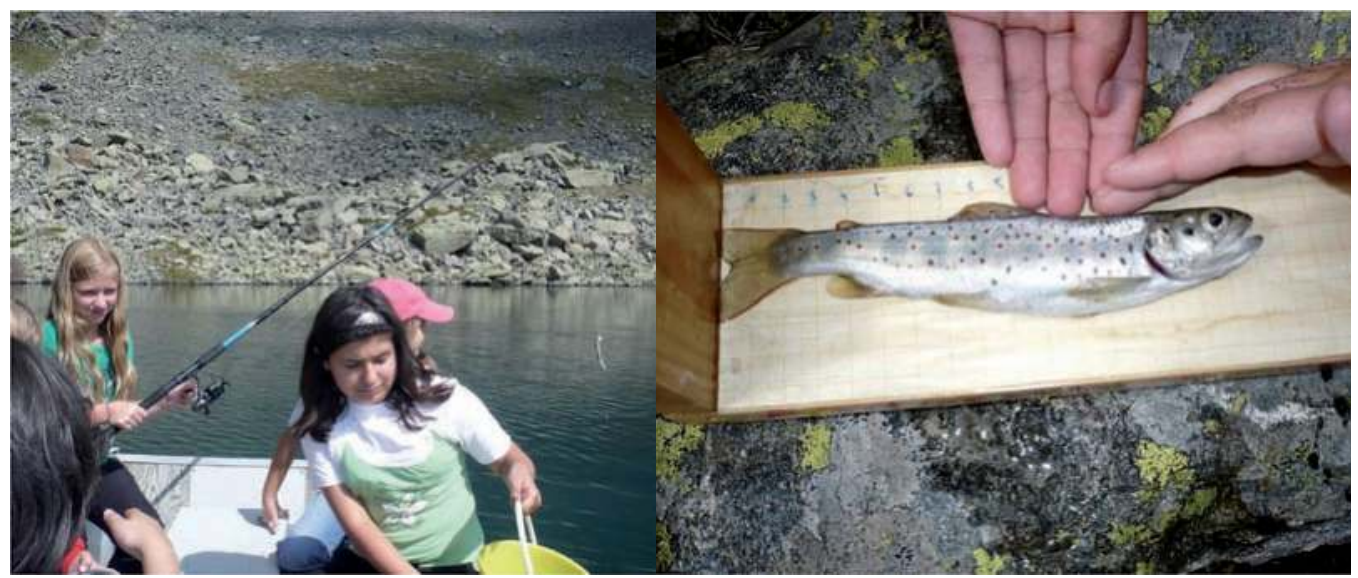

Angeln am Gossenköllesee(links) und vermessen einer Bachforelle (rechts). Fotos: Markus Freiberger 
der Tiere entfernt und tatsächlich fing ein Mädchen nach kurzer Zeit die erste Bachforelle. Diese wurde vermessen und ihr „Konditionszustand“ bestimmt, wie es die Zoologen in ihrem Forschungsauftrag verlangten. Aber warum befanden sich alle Fische knapp über dem Grund. Es musste mit dem Nahrungsangebot zusammenhängen, das war den SchülerInnen rasch klar. Die Limnologin Birgit Sattler fuhr daraufhin mit der zweiten Gruppe hinaus. Ein Planktonnetz befestigt an einer Schwedenrolle wurde zum Grund gesenkt. Nach zwei Fangzügen wurde die Probe verschlossen. Was sich darin befand, sollten die Kinder im Labor erkunden. Unter der Stereolupe fanden die Kinder dann auch Lebewesen. So etwas Ähnliches hatte mancher schon gesehen, der bei einer Kinder-Illustrierten ein Aufzuchtpäckchen mit Salinenkrebsen fand. Es waren ja auch Krebstiere: Ruderfußkrebse, wie die SchülerInnen erfuhren. Einige davon rot gefärbt. Und jetzt lernte auch ein alter Fischer und Biologielehrer etwas dazu: Das Zooplankton trägt in diesem See einen Sonnenschutz, Carotinoide, wie man sie auch bei Schneealgen findet. Und um einen noch besseren Schutz vor der intensiven UV-Bestrahlung in dieser Höhe zu bekommen, lebt das Plankton in größtmöglicher Tiefe.

Alle Ergebnisse wurden dokumentiert. In wenigen Tagen würde man sie präsentieren, und zwar dem Fischexperten von Tirol, Dr. Nikolaus Medygesi, an der Zoologie der Universität Innsbruck.

Forschen und Lernen hatte somit nicht nur etwas mit Daten erheben zu tun. Man muss sie auch dementsprechend präsen-

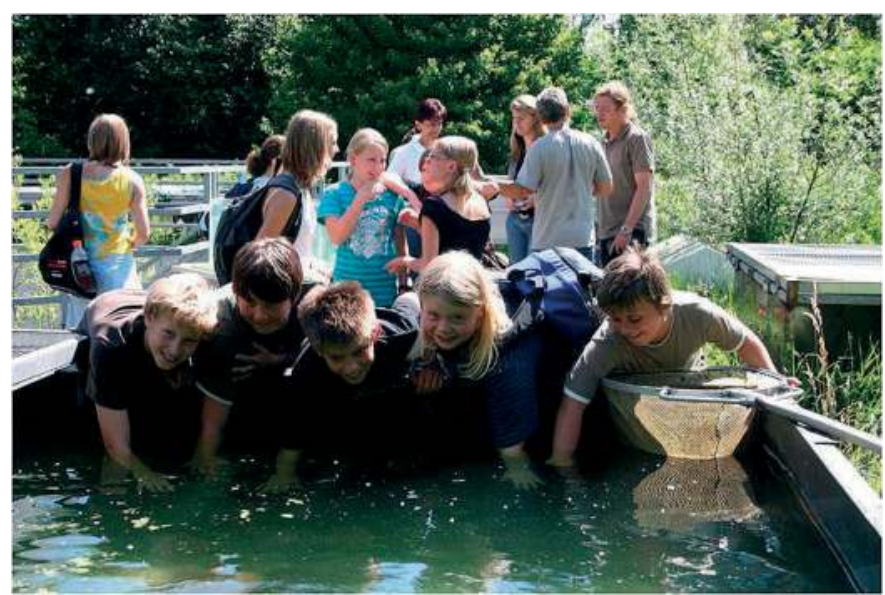

Nacblese der Arbeiten im Freigelände der Universität Innsbruck. Foto: Markus Freiberger tieren können. Doch wer Daten nicht nach außen bringt, hat sie praktisch auch nicht erhoben, darum wurde auch die Anforderung gestellt, zu zeigen, was geforscht wurde. In einem Seminarraum der Universität Innsbruck konnte dies durchgeführt werden, die große Generalprobe für den anstehenden Kinderkongress.

\section{Gletscherschwund und hartes Leben in der Kälte}

\section{Birgit Sattler, Universität Innsbruck}

Herbst 2008: Die SchülerInnen der Hauptschule Zirl befanden sich nunmehr bereits in der 2. Klasse, belastungsfähig genug, so unsere Annahme, sie mit auf den Rotmoosferner im Ötztal zu nehmen. Die Universität Innsbruck verfügt zudem mit dem Universitätszentrum 
Obergurgl mit der dazugehörigen Alpinen Forschungsstelle über eine ausgezeichnete Forschunsgeinrichtung, die den Jungforschern auch Unterkunft bot.

Das Ziel war, im Zuge der Wanderung auf den Gletscher über das Rotmoostal den Gletscherschwund direkt zu sehen. Auf dem Gletscher angekommen, sollten die Kinder die hart-

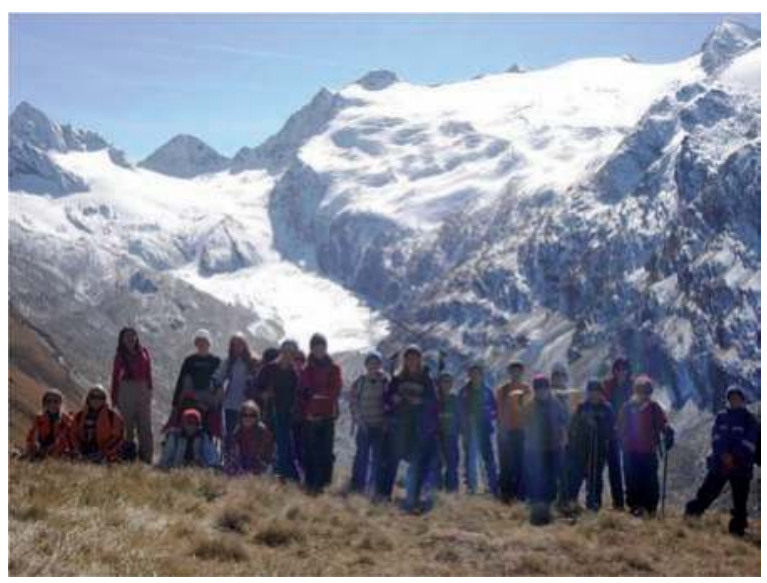

Die Expeditionsmannschaft vor dem Rootmoosferner.

Foto: Birgit Sattler

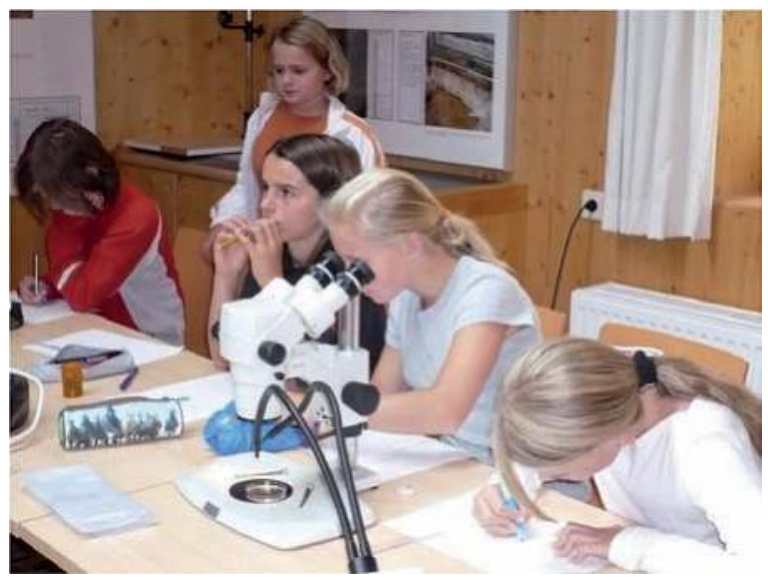

Nachbearbeitung am Universitätşentrum Obergurgl.

Foto: Birgit Sattler näckig überlebende und trickreich an die Kälte angepasste Lebewelt auf dem Gletscher kennenlernen.

Das Ausmaß des Klimawandels erhielt bei vielen Schülerinnen neue Wertigkeiten, als der Gletscherschwund erstmals sichtbar wurde. Die unmittelbare Sichtbarkeit der alten Endmoränen des Rotmoosferners und die bereits mit Vegetation überzogenen alten Gletscherzungen machte das Thema tatsächlich begreiflich. Nicht nur die Präsenz der Tatsache, sondern auch die Geschwindigkeit konnten die SchülerInnen selbst beobachten: Gletscherstände und Eisriegel, welche auf Landkarten relativ jungen Datums eingezeichnet waren, sind in der Natur bereits verschwunden.

Die Natur erobert sich jedoch das eisfrei gewordene Gebiet wieder zurück und so konnten die SchülerInnen selbst beobachten, welche Organismen nun auf dem ehemals vergletscherten Gebiet ihr Terrain eingenommen haben. Doch wie passen sich Organismen an diese harschen Bedingungen im Hochgebirge an? Wie kann eine Insektenlarve in einem eiskalten Gletscherabfluss überleben? Wie kalt ist es dort überhaupt? Dass Lebewesen in derartigen Nischen über spezielle Adaptionen verfügen müssen, versteht sich, um diese aber zu sehen und logisch zu begreifen, dafür mussten die SchülerInnen einige Exemplare aus dem eisig kalten Wasser fangen und lebend zu Tal ins Labor bringen. An der Alpinen Forschungsstelle Obergurgl wurden die mühsam 
erworbenen Fänge mikroskopiert und gezeichnet.

Dieser Arbeitsauftrag war bereits mehr als genug, denn wir mussten aufgrund des fortgeschrittenen Tages auf den Gletscher selbst verzichten.

Larve einer Eintagsfliege (Baetis alpinus) nach der Natur gezeichnet.

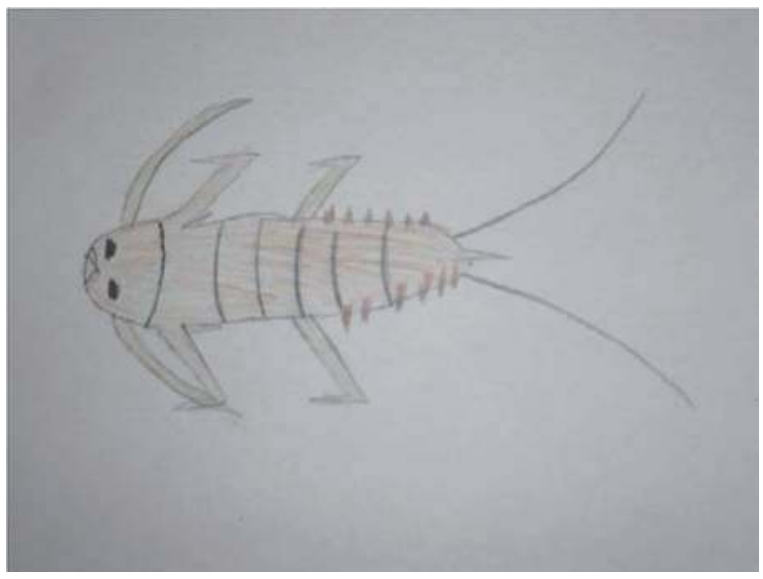

Die Exkursionsteilnehmer im abendlichen Hochgebirge. Foto: Birgit Sattler

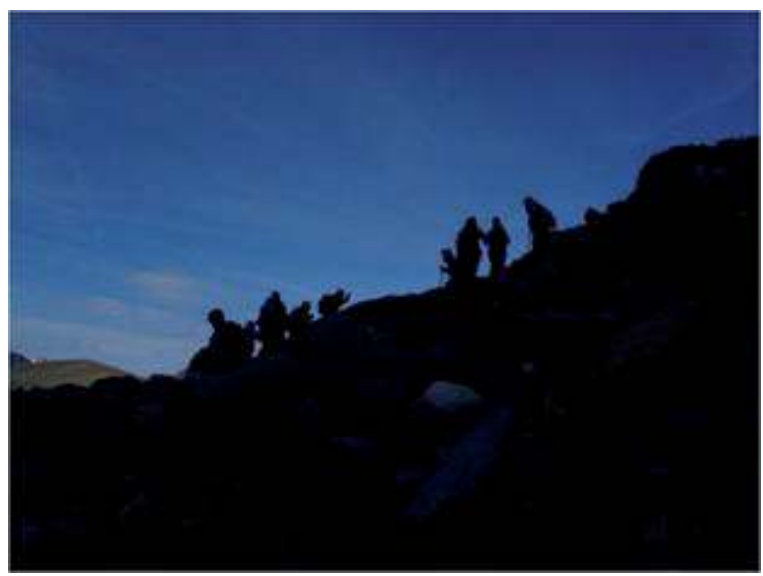

\section{Bärenjagd, Aliens und Extremlebensräume}

Ein Jahr später nahmen wir mit der Klasse den Gletscher nochmals in Angriff, diesmal mit dem Eisbohrer im Gepäck und allem notwendigen Zubehör, um die Eisbewohner auch zu beproben. Diesmal gelang das Vorhaben, die SchülerInnen waren mächtig stolz darauf, zum Teil sogar das erste Mal in ihrem Leben auf einem Gletscher zu stehen. Sie hatten bereits viel Theorie über die mikrobielle Lebewelt im Eis gehört, aber im Zuge von forschendem Lernen wollten die SchülerInnen doch selbst einen Wasserbären fangen.

Allein der Begriff „Wasserbär“ lockte die Kinder, handelt es sich doch um ein Lebewesen, nicht größer als $1,5 \mathrm{~mm}$, welches mit seinen 8 Stummelfüßen drollig aussieht und sogar flüssigen Stickstoff mit $-270^{\circ} \mathrm{C}$ aushalten kann. Ein wahrer Alien, der im Dreck auf dem Gletscher lebt. 


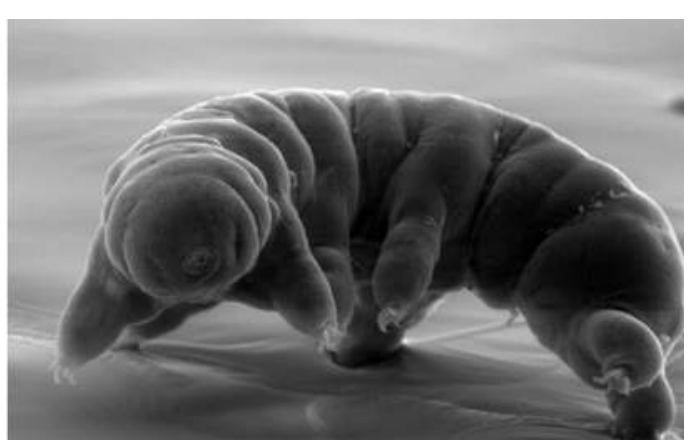

Bärtierchen. Foto: Hieronymus Dastych

Direkt auf dem Eis wurde der Enthusiasmus der SchülerInnen entfacht, es wurden so gennante Kryokonitproben (wörtlich „Eisstaub“) gesammelt, viel vor Ort diskutiert, gefragt, Tondokumente aufgenommen, ertastet und erfahren.

Ihr Klassenzimmer hat sich vom Hochgebirge wieder in das eigentliche verwandelt, wo tatsächlich die Jagd auf Bärtierchen (Tardigrada) verifiziert worden ist. Der König des Überlebens (man kann diese Tierchen auch kochen und sie überleben) hat sich doch noch bei den Kindern vorgestellt.

Das Verständnis der SchülerInnen für Extremlebensräume und den notwendigen Anpassungen konnte somit etwas geschult werden. Auch das Auge für ökologische Nischen. Für die SchülerInnen ist es somit selbstverständlich, dass die Gletscher von Mikroben besiedelt sind.

Es ist für ein Kind oder einen Jugendlichen weitaus weniger interessant, zu hören, wie ein Forschungsprojekt durchgeführt wird, oder wie man als WissenschafterIn zu einem Ergebnis kommt, wie man eine Expedition erlebt und unter welchen Umständen man überhaupt ins Feld gehen muss, um eine Untersuchung durchführen zu können. Wichtig ist es für ein nachhaltiges Verständnis, dass es erlebt werden kann. Dafür mussten wir bei diesem Forschungsthema den Kindern jedoch einiges zumuten. Der Preis, einen kleinen Einblick in den Forscheralltag zu bekommen, war für viele vermutlich hoch. Die 1. Tiroler

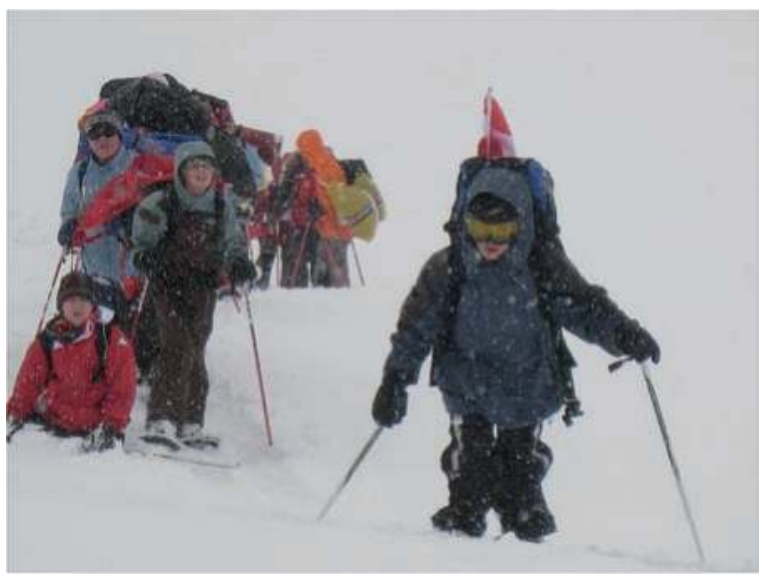

Schülerexkursion auf die limnologische Forschungsstation im Kübtai.
Schülerexpedition auf die limnologische Forschungsstation im Kühtai hat den Kindern sicher viel abverlangt, da sie unter widrigsten Witterungsverhältnissen durchgeführt wurde. Das Arbeiten im Freien im Winter mit bloßen Händen, den Wind im Gesicht, weite Fußmärsche und schweres Gepäck hat den Kindern jedoch ein Gefühl von Solidarität gegeben und auch etwas von Triumph. Sie haben es geschafft, haben es ausgehalten, den inneren Schweinehund bekämpft und schlussendlich war es eine spannende Erfahrung für sie. Durch das eigene Handanlegen wurden Fragen aufge- 
worfen, welche sonst im Trockentraining schwierig zu stellen sind. Der lebendige Bericht zu dieser Expedition ist an anderer Stelle zu lesen.

Ein wichtiger Aspekt bei dieser Art von Projekten ist sicherlich, dass man den SchülerInnen auch zutraut, beispielweise mit Messgeräten umgehen zu können. Ein gewisses $\mathrm{Maß}$ an „Ernst-Nehmen“ fördert die Motivation der Kinder und Jugendlichen. Nach dieser Expedition im Schneesturm auf 2.400m Höhe war weitaus mehr Skepsis seitens der dazugehörigen Erwachsenen vorhanden als von den SchülerInnen selbst. Offensichtlich hatte man ihnen zugetraut, dass sie diesen Aufstieg mit ihrem schweren Gepäck schaffen würden, trotz Sicht gleich Null und Kälte.

Sie waren in diesem Augenblick kleine Polarforscher.

\subsection{Gletscherwoche der 4 C Klasse (8.-12. September 2008) Barbara Bauer-Moser, BRG Kalksburg}

Im Rahmen des BiPolar-Projekts (Biopolar steht für Polarforschung von Wissenschaft und Schule, Hintergrund ist das Internationale Polarjahr (IPY), Bildungskooperation mit Uni Wien und Innsbruck, ZAMG Wien, Schulen aus Wien, NÖ und Tirol) fuhren die 4 C-Klasse mit ihrer Klassenvorständin Prof. Bauer-Moser, mit Prof. Kucera und zwei Eltern ins Rauristal in den Nationalpark Hohe Tauern.

Dort liegt der Gletscher „Goldbergkees“, der von der Zentralanstalt für Meteorologie (ZAMG) wie viele andere Gletscher vermessen wird.

Neben einer botanischen, geologischen und zoologischen Führung durch einen Nationalparkranger des Nationalparks standen vor allem wissenschaftliche Messungen am Gletscher im Vordergrund.

So wurden Pegelstände vermessen, um das Abschmelzen des Gletschers zu dokumentieren, neue Pegelbohrungen von den Schülern mit einem sog. Dampfdruckbohrer durchgeführt und der Gletscherrand mittels GPS bestimmt. Dank der sehr guten Betreung von vier

Raffael Koller und Lukas Gelbmann schreiben in ihrem Protokoll:

„W ir haben Messungen gemacht, wo man siebt, ob der Gletscher an Länge gewonnen oder verloren hat. Das haben wir geschafft, indem wir mittels GPS den Gletscherrand vermessen haben. Alle paar Meter machten wir einen Messpunkt und zeichneten ihn in einer Karte ein. Am Ende sah man an der Karte, wie der Gletscher sich verändert hat.

Der Weg unserer Messung: Wir begannen am Schneefeldende und maßen das Geröllfeld binunter, bis wir zu einem Einsturzbereich kamen, diesen Bereich konnten wir nur ungefähr messen. Als wir beim Messen ans Gletschertor kamen, mussten wir über einen Fluss springen, um zur anderen Gletscherhälfte zu gelangen. Am vierten Punkt erkannten wir, dass der Gletscher eigentlich weit zurückgegangen war. Auf einer Karte trugen wir alle Koordinaten der Messpunk.te ein." 
Mitarbeitern der ZAMG konnten die Kinder einen Blick ins wissenschaftliche, aber auch sehr praktische Arbeiten erfahren. Weiters wurden sie auf das massive Abschmelzen der Gletscher aufmerksam und somit unmittelbare Zeugen der globalen Klimaerwärmung.

\subsection{Flechten - Bewohner extremer Lebensräume Waltraud Buchinger, BRG Englische Fräulein Krems Herwig Zeiler, BRG Englische Fräulein Krems}

In Ermangelung von Schnee, Eis, benachbarter Hochgebirge und Gletscher in der Region um Krems war es schwierig, Versuche direkt mit Schnee und Eis bzw. Exkursionen durchzuführen. So suchten wir im Rahmen dieses Projektes andere Betätigungsfelder.

Die extremen Lebensbedingungen der Polarregionen kristallisierten sich als zentrales Thema heraus und im fächerübergreifenden Biologie- und Physikunterricht entwickelten sich verschiedene Projektideen.

- Versuche zur Thermoregulation der Polartiere (Biologie und Ökologie)

- Erarbeiten von pflanzenphysiologischen Experimenten für die Polarregionen (Biologie und Ökologie)

- Entwicklung und Selbstbau von einfachen Licht- und Temperaturmessgeräten (Physik)

- $\quad$ Flechten als Bewohner extremer Lebensräume: Projekttage mit Univ. Prof. Dr. Roman Türk (Biologie und Ökologie)

\section{Versuche zur Thermoregulation der Polartiere: Schülerexperimente}

Nach ausführlichen Besprechungen über die extremen Standortfaktoren der Polarregionen erhielten die SchülerInnen als Vorgabe nur verschiedene Materialien für die Durchführung

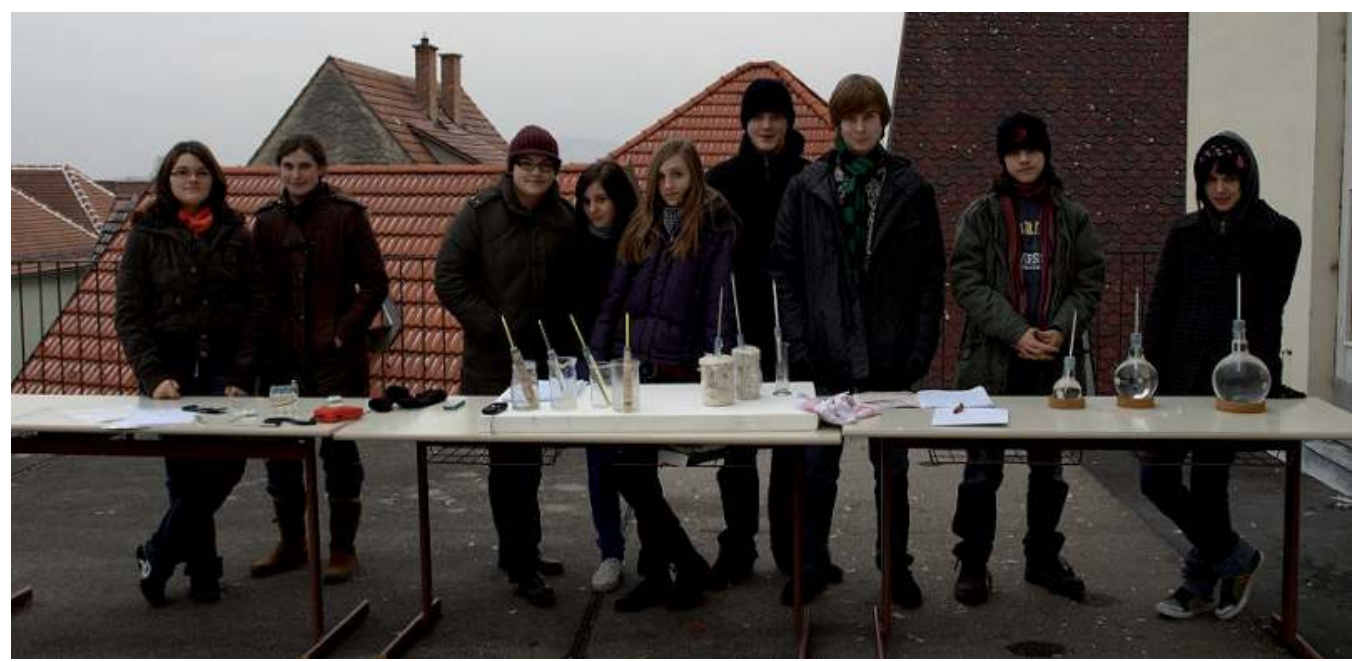

Durchführung der Experimente zur Thermoregulation bei Tieren im Freien bei minus $3{ }^{\circ} \mathrm{C}$.

Foto: Waltraut Buchinger 


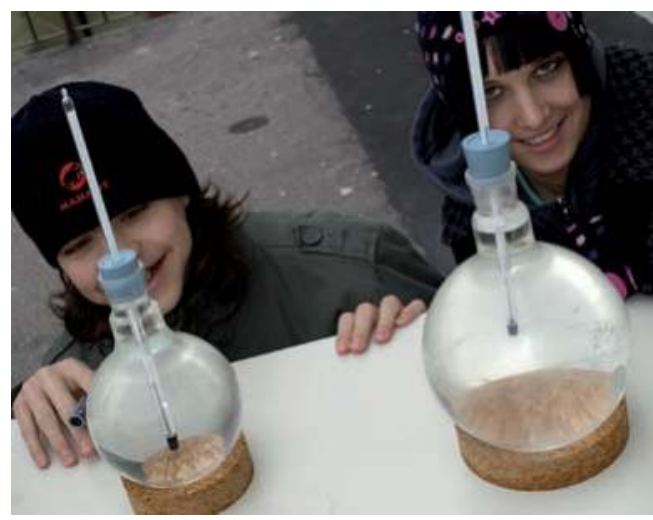

Versuch zum Nachweis der Bergmann'schen Regel: Größenabhängigkeit des Wärmeverlustes.

Foto: Waltraut Buchinger

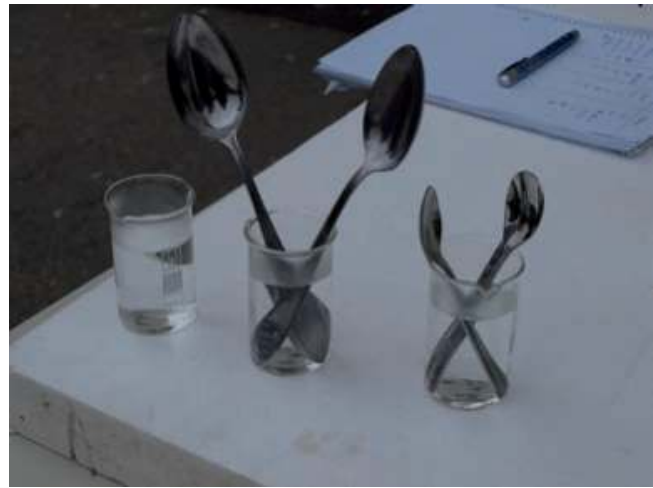

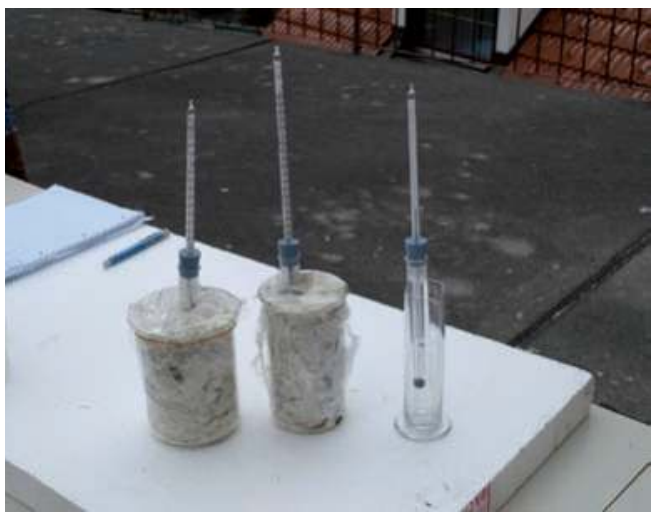

Isolationsversuch mit verschieden dicht gepackten Federn. Foto: Waltraut Buchinger

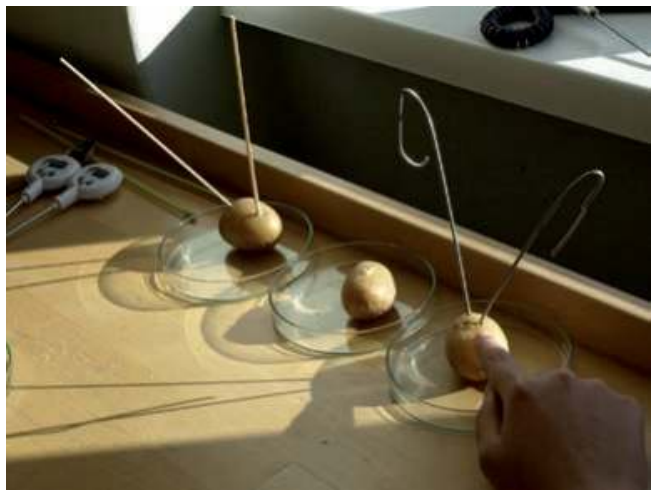

Versuche zum Nachweis der Allen'schen Regel: Temperaturableitung durch Extremitäten. Fotos: Waltraut Buchinger

Sophie-Anna Melichar, 6c:

„Nur mit Hilfe vorgegebener Materialien entwickelten wir diverse Experimente mit Modellorganismen, die die verschiedenen Anpassungen an die extreme Kälte der Polarregionen widerspiegeln sollten. Die Modellorganismen stellten mit warmem Wasser gefüllte Glasgefäße dar.

Um nur annähernd arktische Verbältnisse nachzuahmen, führten wir die Versuche dann auch im Freien durch, bei minus $3{ }^{\circ} \mathrm{C}$.

Die Versuchsergebnisse wurden genauestens protokolliert und vor der Klasse in Form einer Powerpoint-Präsentation dargebracht. Die Protokolle wurden beim KINKONG (Kinderkongress) an der Universität Wien aufgelegt.

Das Polarprojeket hat mir sehr gut gefallen und es war toll, anhand von Versuchen selbstständig so viele Informationen herauszufinden. Ich bin froh, dass gerade unsere Klasse die Möglichkeit hatte, an diesem Projekt zu arbeiten." 

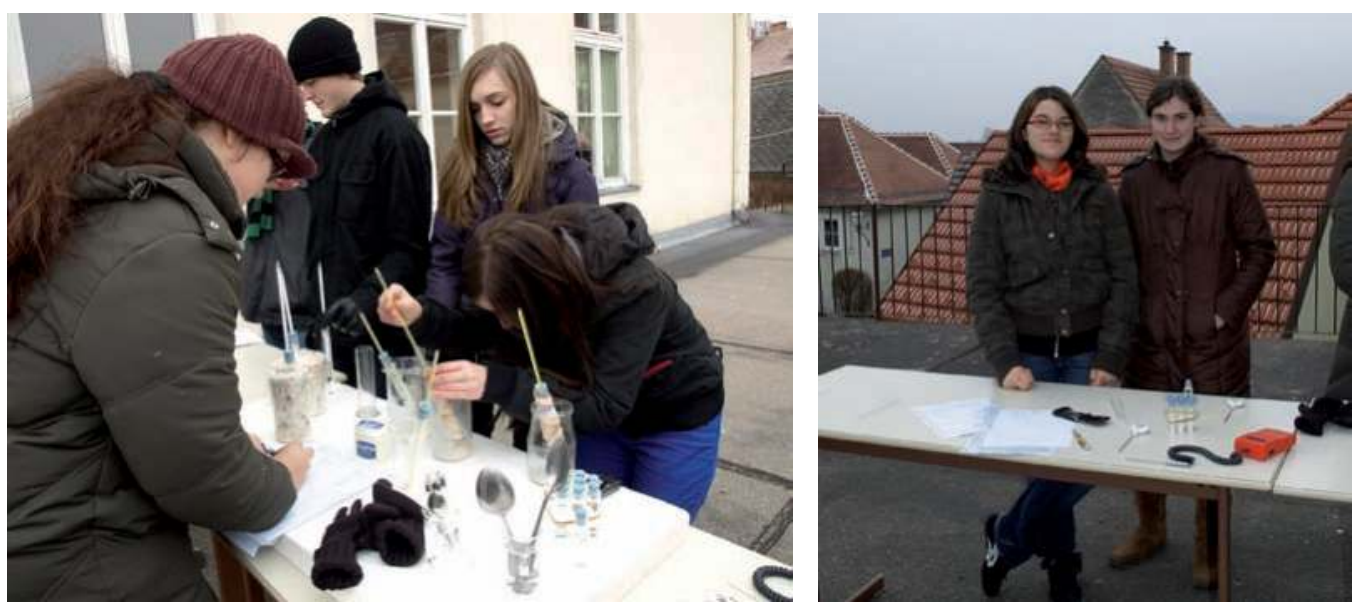

Isolationsversuche mit Fett und der Versuch zur Reduktion des Wärmeverlusts in der Gruppe. Fotos: Waltraut Buchinger

eines Experimentes. In Gruppenarbeit wurde der Ablauf der verschiedenen Versuche selbstständig geplant sowie eine Hypothese über den Ausgang der Experimente aufgestellt. Die Versuche wurden genau protokolliert und in der Klasse präsentiert.

\section{Entwicklung und Selbstbau von einfachen Licht- und Tempera- turmessgeräten}

\section{Beschreibung der Messgeräte:}

Zur Lichtmessung werden Fotodioden des Typs BPW 34 verwendet. Mittels Fotodiode und eines passenden Widerstandes wird dabei ein einfacher Stromkreis aufgebaut. Der
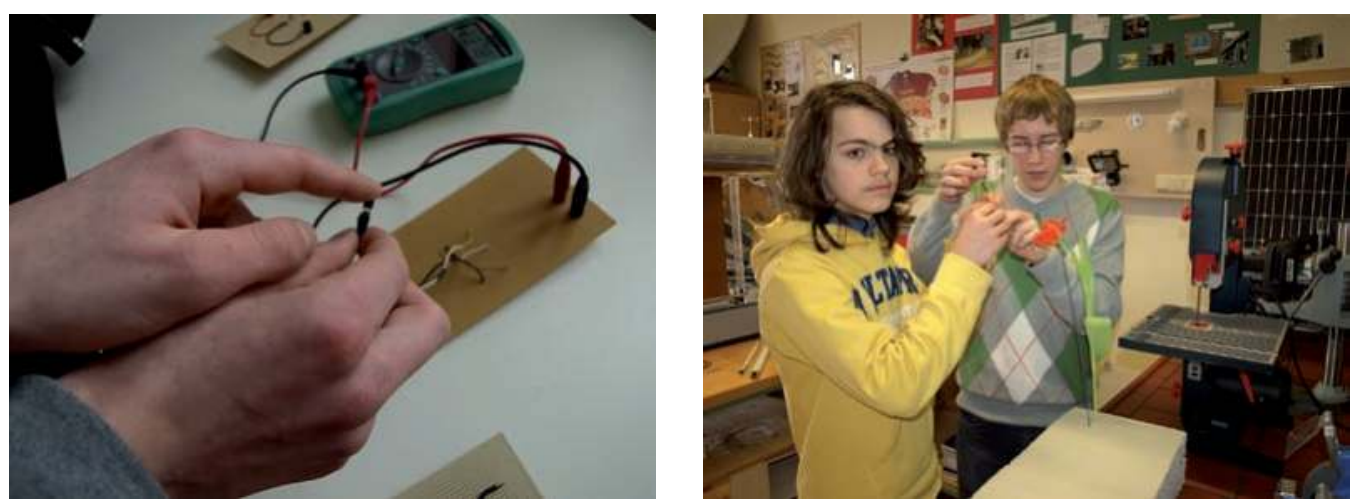

Herstellung der Messgeräte und Anbringen der Messsonden an der Blüte. Fotos: Waltraut Buchinger 
Bernadette Löffler, 6C:

„In Biologie erarbeiteten wir Versuchsanleitungen für Licht- und Temperaturmessungen im Blütenbereich von arktischen Pflanzen und ibrer Umgebung. Wir möchten herausfinden, ob in der Blüte arktischer Pflanzen eine böhere Temperatur und ein höherer Lichteinfall zu messen sind als außerhalb der Blüte. Im Physikunterricht mit Professor Zeiler überlegten wir die praktische Umsetzung und stellten die Messgeräte her.

In Kleingruppen diskutierten wir eifrig, wie man am besten die Sensoren anbringen kann, obne die Pflanze zu zerstören. Dabei waren wir uns vorerst keineswegs einig, doch in der Praxis wurde rasch eine Lösung gefunden.

Wer jetz̨t denkt, dass die Herstellung von unseren Geräten einfach war, hat sich getäuscht, wir mussten bohren und löten, aber dennoch konnte man Professor Zeiler und uns am Strablen der Augen ansehen, wie viel Freude uns die Arbeit bereitete.

Unsere Meinung: Physik sollte immer so sein!"
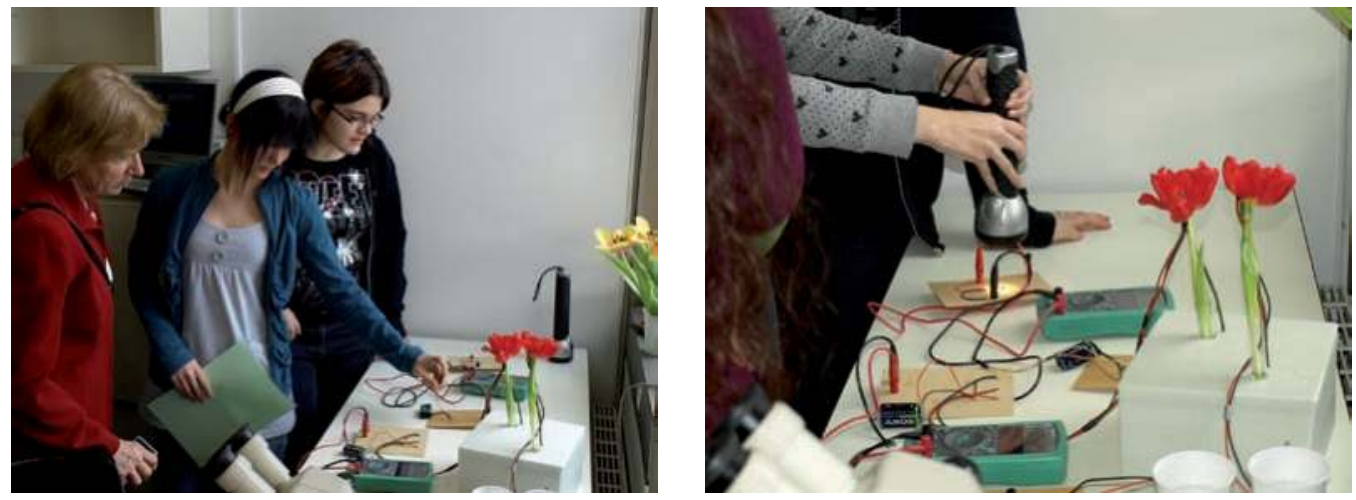

Präsentation unserer Messgeräte beim Kinderkongress (KINKONG) vor interessierten ScbülerInnen und LebrerInnen. Fotos: Waltraut Buchinger

Spannungsabfall am Widerstand wird in Folge mit einem Multimeter gemessen.

Nun muss noch eine Eichung der Apparatur mittels Sternpyranometer erfolgen, was noch aussteht. Die Strahlungsintensität wird bei Pyranometern nicht unmittelbar, sondern auf dem Umweg über eine durch sie erzeugte Temperaturdifferenz gemessen. Diese wird mittels Thermoelement in eine proportionale Spannung umgesetzt. Die Uni Wien wird dieses Gerät zur Verfügung stellen. Messungen an der Uni Wien (im Rahmen meiner Diplomarbeit) haben ergeben, dass die Unge-

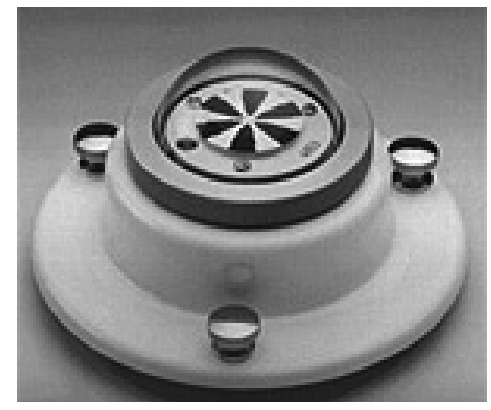

Sternpyranometer 


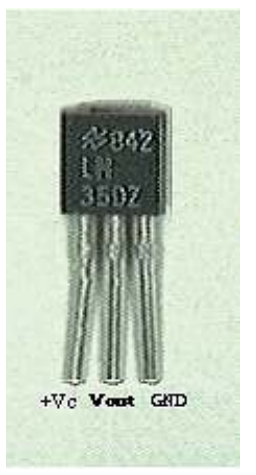

nauigkeit der Fotodiode als selektiver Absorber bei maximal 5\% liegt, was für einen derart kostengünstigen Versuchsaufbau beachtlich ist.

Zur Temperaturmessung werden fertige Sensoren des Typs LM 35 verwendet. Sie sind mit einer Spannung von 9V zu versorgen, was mittels Blockbatterie möglich ist. So muss man nur mehr eine Anschlussmöglichkeit für ein Voltmeter schaffen, was wir über eine Platine realisierten, auf der zwei Steckkontakte angebracht wurden, welche mit den Kabeln, die zum Sensor führen, verlötet wurden. Auf dem Voltmeter liest man so einen Spannungswert ab, der der herrschenden Temperatur entspricht: z.B. $238,3 \mathrm{mV}$ sind $+23,83^{\circ} \mathrm{C}$.

\section{Der Temperatursensor LM 35}

\section{Flechten als Bewohner extremer Lebensräume}

Projekttage mit Univ. Prof. Dr. Roman Türk

Die Frage nach der Existenz von Leben in Regionen mit lebensfeindlichen Bedingungen brachte die Flechten ins Spiel. Zu Beginn konnten sich die SchülerInnen darunter nur sehr wenig vorstellen. Im Zuge unserer Aktivitäten aber haben sie die Flechten als faszinierende Lebewesen kennen gelernt.

Dr. Roman Türk, Professor für Pflanzenökologie an der Universität Salzburg verbrachte zwei Projekttage mit unseren SchülerInnen.

Er begeisterte sie mit einer eindrucksvollen Powerpoint-Präsentation über die Flechtenforschung in der Antarktis und die Arbeit der Lichenologen (Flechtenforscher). Die SchülerInnen lernten dabei die Flechten auch als Bewohner der lebensfeindlichsten Regionen kennen. Ein Vortrag über die Biologie und Ökologie der Flechten ließ die SchülerInnen rasch erkennen, warum diese Symbiose zwischen Alge und Pilz Vorteile beim Überleben bringt.
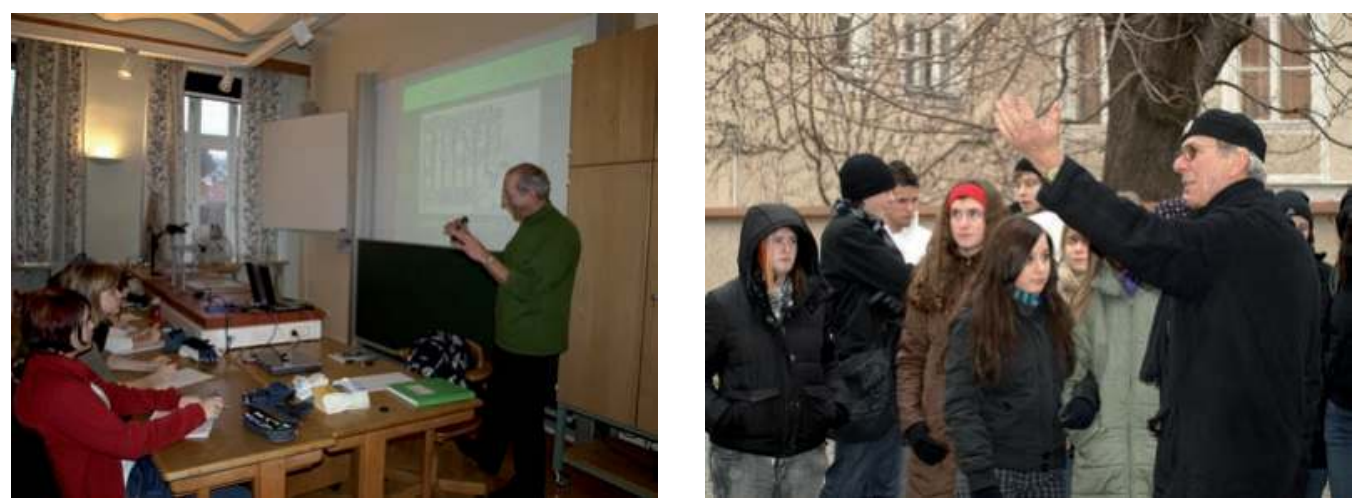

Prof. Türk beim Unterricht in der Schule und in der Umgebung. Fotos: Waltraut Buchinger 
Eine Exkursion in die Umgebung der Schule und auf den Kreuzberg mit seiner Weingartenlandschaft machte die praktische Auseinandersetzung mit diesen Lebewesen möglich. Ausgerüstet mit Lupen, Taschenmessern und kleinen Säckchen sammelten die jungen Flechtenforscher verschiedene Flechtenarten. Das Entnehmen der Flechten von den Ästen
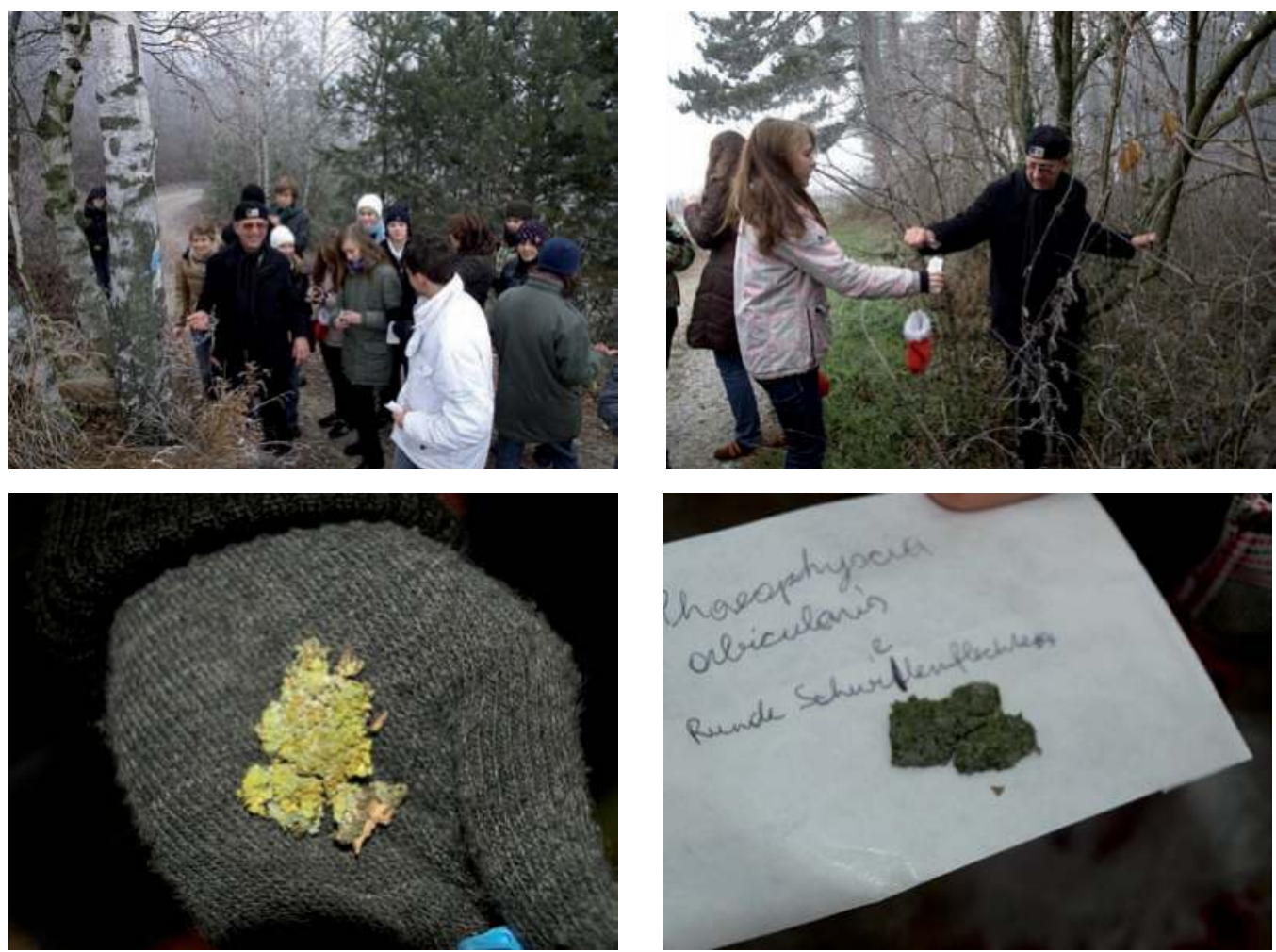

Exkursion auf den Kreuzberg. Fotos: Waltraut Buchinger

mit Hilfe eines Taschenmessers erforderte etwas Geschick. Die von Dr. Türk bestimmten Flechten wurden sofort in die kleinen Säckchen verpackt und sorgfältig beschriftet, wobei die korrekten Artbezeichnungen für Verwirrung sorgten.

Prof. Türk konnte zeigen, dass extreme Bedingungen nicht nur in Hitze- oder Kältewüsten wie den Polarregionen oder im Hochgebirge zu finden sind. Auch in unseren Breiten kommen Kleinlebensräume wie Gesteinsoberflächen, Mauern, Dächer oder Borken der Bäume vor, die ebenfalls Extremstandorte darstellen. Dort herrschen Mangel an Wasser und nährstoffreichem Boden und je nach Ausrichtung auch extreme Temperaturen, im Winter große Kälte und im Sommer oft große Hitze.

In der Schule wurden die Funde nochmals gesichtet und Dr. Türk demonstrierte das Anlegen und Beschriften eines Flechtenherbariums. Das anschließende Mikroskopieren der 
verschiedenen Flechtenfunde brachte faszinierende Formen und Gestalten zum Vorschein. Dabei erfuhren die SchülerInnen, worauf es beim genauen Bestimmen von Flechten ankommt und, dass die Bestimmung nur nach dem äußeren Erscheinungsbild nicht ausreicht. Für den KINKONG an der Universität Wien erarbeiteten die SchülerInnen einen Vortrag zum Thema Flechten als Bewohner extremer Lebensräume. Darin veranschaulichen sie verschiedene biologische Aspekte der Flechten und versuchen Interesse für diese oft unbemerkten Lebewesen zu wecken und erläutern ihre Bedeutung im Naturhaushalt.

Auszug aus der Powerpoint-Präsentation „Flechten - Bewohner extremer Lebensräume“ der 6C:

Interessant ist auch die Rolle der Flechten als Bioindikatoren. Sie sind sehr empfindlich gegenüber den Schadstoffen der Luft. Das Vorkommen bestimmter Flechtenarten deutet auf saubere Luft hin. Feblen diese Lebensformen weitgehend, so ist das ein Hinweis auf Luftverschmutzung.
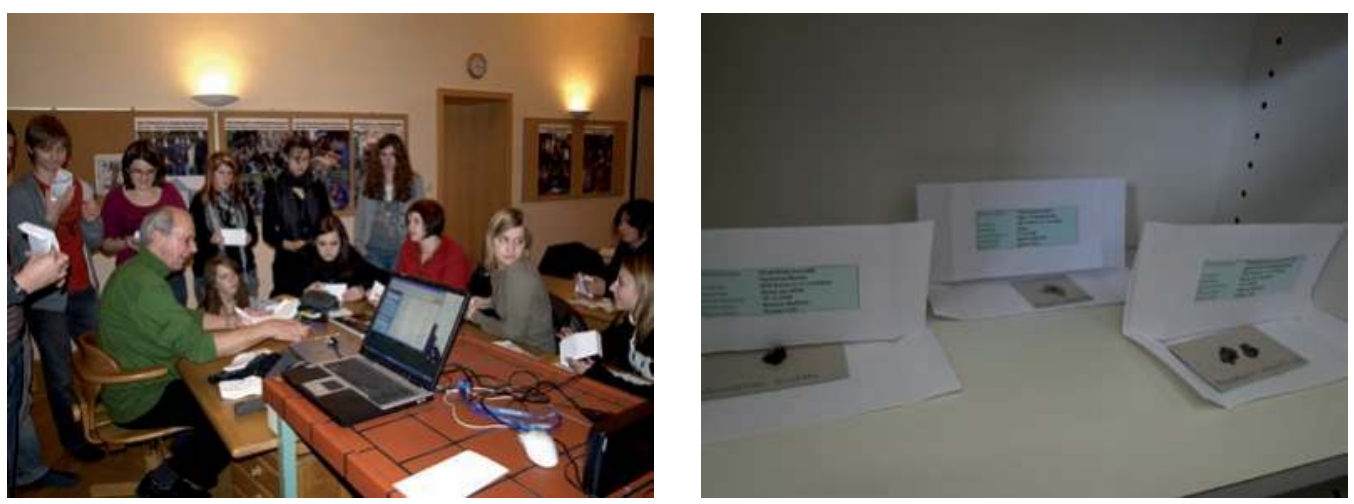

Anlegen eines Flechtenherbariums. Fotos: Waltraut Buchinger

Leider mussten wir beim Sammeln der Flechten zu einem ernüchternden Ergebnis kommen, unsere Ausbeute war äußerst gering. Die alte Linde auf unserem Schulgelände sollte voll sein mit Flechten, wir konnten aber keine einzige finden. Die Situation besserte sich ein wenig, als wir in den Weinbergen suchten. Wir konnten nur neun verschiedene Flechtenarten sammeln. Normalerweise hätte man über 20 verschiedene Arten erwarten dürfen.

So ist eine Fortsetzung des Projektes in Planung. Dr. Roman Türk hat sich bereit erklärt, für weitere Flechtenkartierungen an unsere Schule zu kommen und die Luftgütesituation in Krems zu erforschen. 


\section{Kapitel 4}

Forscherlnnen der Zukunft (Scientists of the Future) 



\title{
4. ForscherInnen der Zukunft (Scientists of the Future)
}

\begin{abstract}
„Im dritten Modul sollen die mit der Wissenschaft gemachten Erfahrungen insofern vertieft werden, als die SchülerInnen gemeinsam mit den LehrerInnen kleine wissenschaftliche Aufgabestellungen definieren, welche von den WissenschaftlerInnen vor Ort in der Arktis bearbeitet werden sollen. Die Entwicklung der Projektideen sowie deren Umsetzung (Methodik und Erprobung derselben) sollen von den SchülerInnen in Kooperation mit den LehrerInnen durchgeführt werden. Im Falle von BIPOL AR werden die Tätigkeiten der Wissenschafter audiovisuell (Video, Foto und Tondokumente) dokumentiert, um die Anschaulichkeit zu verbessern. Eine Koordination und direkte Absprache mit den „Jungforschern" über Satellitentelefon oder E-Mail, eventuell auch mit Hilfe neuer Medien (Weblogs) ist geplant. Die erhobenen Daten und Ergebnisse der Experimente brw. fallweise Proben werden im Anschluss gemeinsam mit den SchülerInnen bearbeitet und ausgewertet. "
\end{abstract}

\subsection{Projektideen der SchülerInnen Michaela Panzenböck, BG/BRG Lilienfeld}

Soweit die im Projektantrag formulierte Idee. Die Umsetzung - v.a. die Entwicklung der Projektideen - gestaltete sich schon schwieriger. Dies lag im Wesentlichen daran, dass sowohl SchülerInnen als auch LehrerInnen keine wissenschaftliche Erfahrung bzw. Vorstellung über die Durchführbarkeit mancher Projektideen haben. Zwei dennoch erfolgreich durchgeführte, weitgehend selbständig von SchülerInnen und LehrerInnen bearbeitete Forschungsfragen werden im Anschluss vorgestellt.

Ein weiterer Forschungsauftrag lautete, die Geräusche der Arktis „einzufangen“. Dieses von Wissenschaftern produzierte Tondokument wurde in weiterer Folge von SchülerInnen geschnitten und beim Kinderkongress in der Lauschecke abgespielt.

Ein Weblog, der auch die Gletscherexkursionen der Schulen beinhaltete, ermöglichte SchülerInnen und LehrerInnen, die Expeditionen der Wissenschafter in die Arktis und Antarktis doch ein wenig mitzuerleben (www.sparklingscience.at/blog). Ein Highlight war auch der telefonische Kontakt zu der Antarktisexpedition 2008. Per Satellitentelefon konnten die SchülerInnen der HS Zirl ihre brennenden Fragen über das Leben und Forschen am anderen Ende der Welt direkt an Michael Storrie-Lombardi (Kinohi Institute, USA), Chris McKay (NASA Ames) und Art Mortvedt (Buschpilot aus Alaska) stellen.

\subsection{Schlaflos in der Arktis? - Eine Biorhythmusstudie Michaela Panzenböck, BG/BRG Lilienfeld}

Welchen Einfluss hat die Tageslänge auf die Schlafgewohnheiten von Menschen? Diese Frage untersuchte eine Schülergruppe des BG/BRG Lilienfeld im Rahmen von BIPOLAR. Nachdem die lichtsensible Zirbeldrüse über die Melaninausschüttung das Schlafverhalten 


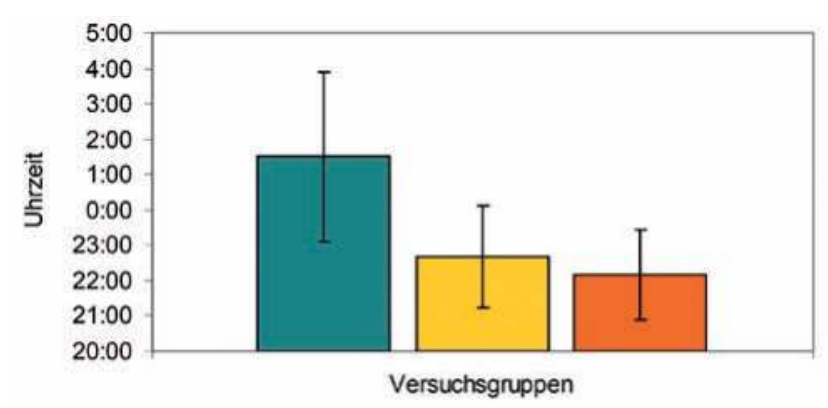

$\square$ Versuchsgruppe Arktis $\square$ Kontrollgruppe Winter $\square$ Kontrollgruppe Sommer

Einschlafzeit der befragten WissenschaftlerInnen während der Polarexpeditionen im Sommer 2008.

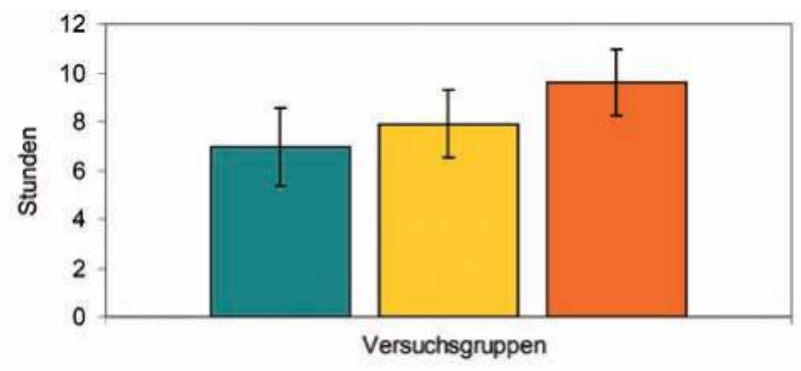

$\square$ Versuchsgruppe Arktis $\square$ Kontrollgruppe Winter $\square$ Kontrollgruppe Sommer

Durchschnittliche Schlafdauer der befragten WissenschaftlerInnen während der Polarexpeditionen im Sommer 2008.

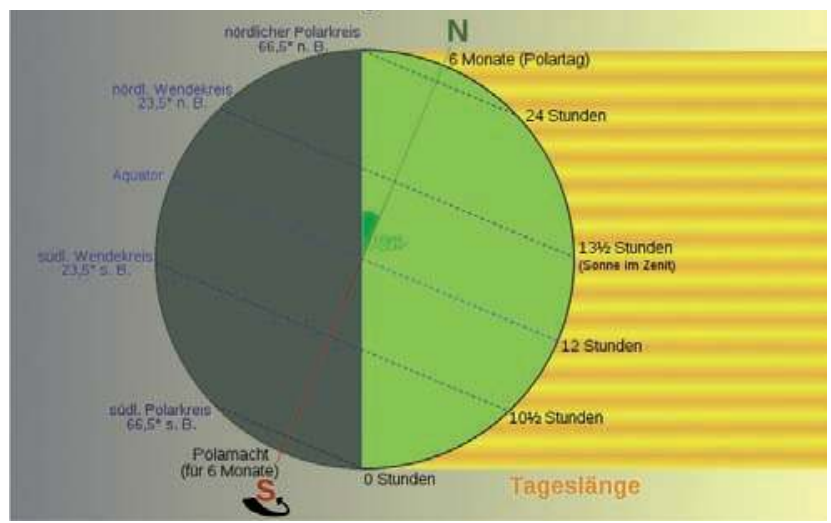

reguliert, ist beim Vierundzwanzig-Stunden-Tag des arktischen Sommers eine höhere Aktivität der Menschen bei geringerem Schlafbedürfnis anzunehmen. Um dem nachzugehen entwickelten SchülerInnen einen Fragebogen, in den Forscher und andere Bewohner der Forschungsstationen in Grönland, in Abhängigkeit von Geschlecht und Alter ihre Schlafgewohnheiten eintragen mussten. Eine Kontrollgruppe in den gemäßigten Breiten, bestehend aus Verwandten und Freunden der SchülerInnen, musste denselben Fragebogen ausfüllen. Die Daten wurden anschließend ausgewertet, analysiert und unter dem Titel „Schlaflos in der Arktis?" ebenfalls beim Kinderkongress (KinKong) präsentiert. Zusammenfassend lässt sich bei Personen, die sich in permanentem Licht aufhalten, eine geringere Schlafdauer gegenüber der Kontrollgruppe feststellen. Noch viel auffälliger ist, dass sie später schlafen gehen als Menschen in Breiten, wo die Sonne untergeht.
Veranschaulichung der (Beleuchtung) am 21. Juni.

Tageslänge 


\subsection{Verrottungsversuch des BRG 6 Marchettigasse Bettina Girschick, Elfriede Schmid, Elisabeth Fuchs BRG 6 Marchettigasse Wien}

Im Rahmen des BIPOLAR-Projekts wurden die Schülerinnen und Schüler des BRG 6 Marchettigasse ermuntert, Fragen an die Forscherinnen und Forscher zu stellen, d.h. sie durften ihnen „kleine“ Forschungsaufträge erteilen. Diese Forschungsaufträge wollten die Wissenschafter dann vor Ort, in Grönland, durchführen.

Unsere Schülerinnen und Schüler wählten sich das Thema „Verrottung““ als Forschungsgegenstand. Sie beabsichtigten gemeinsam mit den Wissenschafterinnen und Wissenschaftern einen diesbezüglichen Verrottungsversuch durchzuführen:

Dazu wurden schon Anfang Juli ein Filterpapier, etwas Kunststoff auf Stärkebasis und ein Stück eines Polylactatbechers einer Fast Food Kette in Gartenerde eingegraben (Foto 4.3.3). Nach sechs Wochen sollte untersucht werden, wie weit die Verrottung fortgeschritten war (Foto 4.3.4).

In Grönland wurde dieser Versuch im selben Zeitraum ebenfalls durchgeführt: Dankenswerterweise hatte Mag. Michaela Panzenböck unsere Probensackerl in die Arktis mitgenommen und dort eingegraben, was unter arktischen Bedingungen gar nicht so einfach ist. Es klingt zwar kurios, aber unter diesen Bedingungen wird sogar das Wiederfinden zum Problem. Denn die Vergrabungsstelle musste ein sehr markanter und charakteristischer Platz sein. Wie sollte denn sonst die Person, die das Sackerl nach sechs Wochen wiederum auszugraben hatte, dieses auch tatsächlich finden?
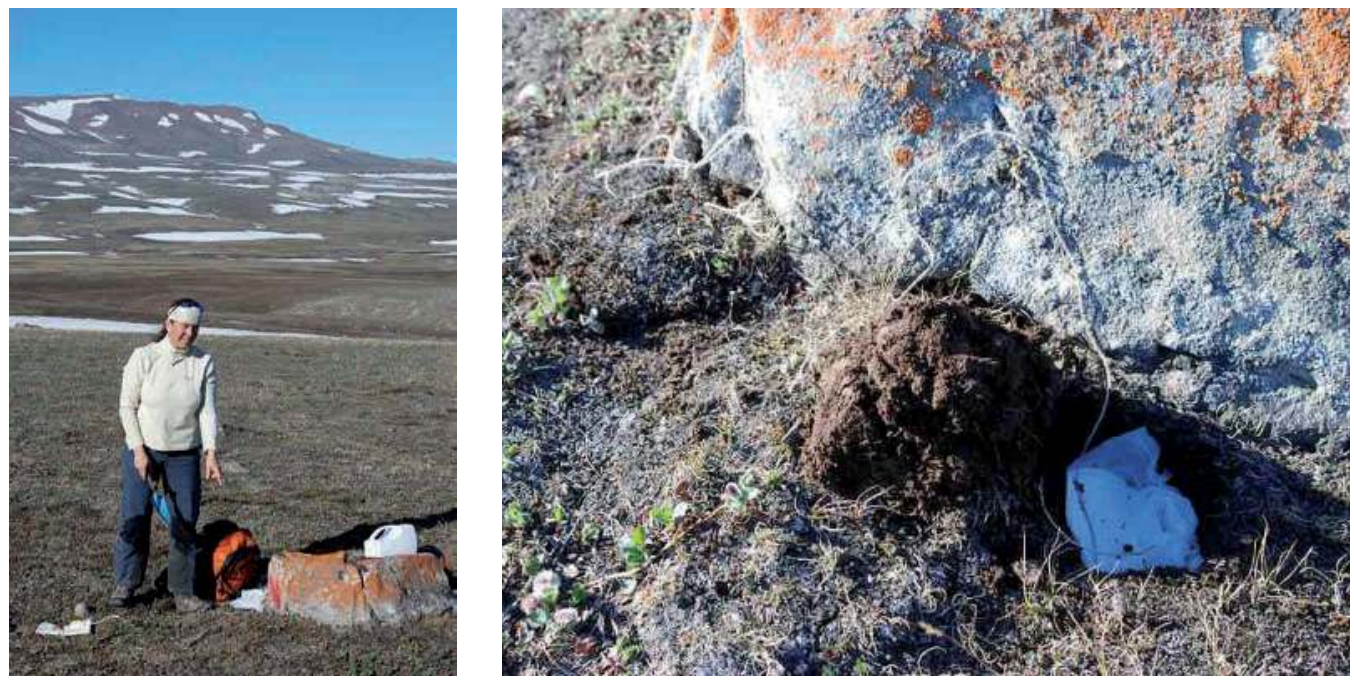

Standort für den Verrottungsversuch in Grönland. 
DI Gernot Weyss zeigte Spürsinn: Er entdeckte den markanten Stein über der Vergrabungsstelle und brachte uns die Proben zur Auswertung wieder nach Österreich mit.

Das Ergebnis unseres Verrottungsversuches war folgendes:

Nach sechs Wochen war das Filterpapier im Wiener Garten ganz verrottet, auch der Kunststoff auf Stärkebasis zeigte schon einige Zerfallserscheinungen. Der Masseunterschied betrug 0,07 Gramm. Dies entspricht immerhin einem Abbau von 15 \%. Der Polyacetatbecher war unberührt geblieben.

In Grönland war nicht einmal beim Filterpapier ein Zerfall feststellbar. Bei diesen tiefen Temperaturen verlaufen Verrottungsvorgänge offensichtlich viel langsamer. Ein beeindruckender Beweis für unsere Vermutungen, der uns mit Unterstützung der Wissenschafter vor Ort gelungen ist.
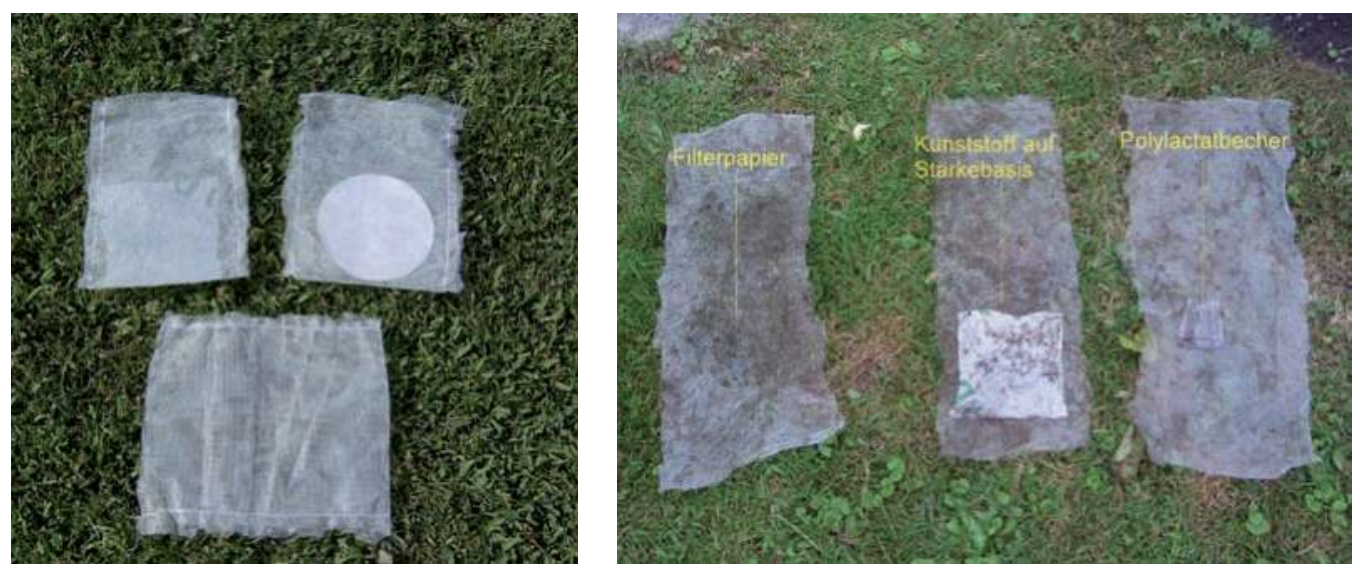

Gazetaschen für den Verrottungsversuch. Links vor der Vergrabung, rechts nach sechs Wochen.

\subsection{KINKONG (der Kinderkongress in Wien)}

Ein wichtiger Teil im Leben eines erfolgreichen Wissenschafters ist neben der eigentlichen Forschungsarbeit die Veröffentlichung seiner Forschungsergebnisse, unter anderem deren Präsentation vor Fachkollegen im Rahmen von Kongressen. Um die SchülerInnen auch diesen Aspekt des Forscheralltags erleben zu lassen, wurde ein Kinderkongress mit dem klingenden Namen KINKONG veranstaltet. Am 20. Februar 2009 fanden sich 170 SchülerInnen unserer „,coolen“ Schulen aus ganz Österreich begleitet von 22 LehrerInnen und Eltern und 7 WissenschafterInnen am Biozentrum der Universität Wien ein, um im großen Hörsaal die Ergebnisse ihrer Projektarbeiten zu präsentieren. Eröffnet wurde die Veranstaltung vom Leiter des Departments für Limnologie und Hydrobotanik, Prof. Dr. F. Schiemer, und Ministerialrat Dr. C. Smoliner vom BMWF, mit der Moderation wurden zwei Schülerinnen des BG Bachgasse, Mödling, betraut. Zwischen den Vorträgen der JungwissenschafterInnen konnte an zahlreichen, von den Jugendlichen betreuten Stationen experi- 
mentiert oder den Geräuschen der Arktis gelauscht werden. Selbstentworfene KINKONGSticker fanden ebenso ihre Abnehmer wie es Besucher zu den Mikroskopen hinzog, mit deren Hilfe Gletscherflöhe und Flechten en detail betrachtet werden konnten. Parallel dazu fand im Hörsaal eine Vorführung der von den SchülerInnen produzierten Kurzfilme statt. Internationale Beteiligung in der Person von Dr. M. Storrie-Lombardi, USA, mit seiner „Lasershow“, einer Methode zum Auffinden organischer Substanz in Schnee und Eis, rundete einen gelungenen Kinderkongress ab.

„Zuletzt durften wir noch zum KinKong (Kinder Kongress) im Institut für Ökologie in Wien fahren, wo wir unsere Arbeit präsentierten und uns die Arbeiten der anderen Klassen ansehen konnten. Wien war für mich ein absolutes Highlight, da der KinKong sehr gut organisiert war und es für mich sehr interessant war zu sehen, was andere sich zu diesem Projekt überlegt hatten. Und natürlich freuten wir Schüler uns sehr nach Wien zu fahren, weil es eine tolle Abwechslung vom Schulalltag war."

Lisa Gamper

„Spaß hat auch die Schlusspräsentation gemacht, wir waren nengierig auf die Ergebnisse der anderen Schulen. Genauso konnten wir beim KinKong auch Kontakte zu anderen Schulen knüpfen, die Tage in Wien waren jedenfalls eine schöne Zeit."

Thomas Hirn

„Wenig darauf folgte unser letzter Projekttag vor dem KinderKongress in Wien. An diesem Tag stellten wir unsere Arbeiten fertig und bereiteten uns auf den Kongress vor.

In Wien verbrachten wir zwei Tage, wo wir unser Projekt beim KinKong vorstellten. Vom KinKong bin ich ein wenig enttäuscht; zeitweise war es sehr langweilig, da alle Filme zweimal gezeigt wurden und an den Ständen nicht viel Interesse herrschte. Für ein nächstes solches Projekt könnte man so einen Kongress vielleicht besser organisieren und gestalten.

Vielleicht könnte man die Öffentlichkeit mehr über solche Projekte informieren, so dass interessierte Leute sich den KinKong und die Präsentationen der einzelnen Projekte ansehen könnten. Auch ein intensiverer Kontakt mit Wissenschaftler wäre schön gewesen. "

Veronika Kinzner

„Mit Unterstütrung der Fachlehrer aus Geographie, Geschichte, Biologie und Englisch standen wir schließlich am 19.2.2009 mit einer Power-Point-Präsentation in der Laptop-Tasche sowie einer Homepage im Web am Innsbrucker Hauptbahnhof und warteten auf unseren Zug zum KinKong (Kinder Kongress) des ökologischen Instituts der Uni Wien.

Trotz. mehrstündiger Verspätung unseres Zuges und einem kurafristigen Verlegen auf den Schienenersatzverkehr kamen wir schließlich doch in Wien an. Am 20.2.2009 konnten wir schließlich um ca. 11 Uhr vor einem vollen Saal unseren Vortrag halten. Leider begleitet von einer kleinen Katastrophe, nämlich der falschen, nicht korrigierten, Version unserer Präsentation, beendeten wir so unser Projekt und können stolz sein, sicherlich eines der Highlights des Kongresses gewesen zu sein. "

Fabian Laimer 

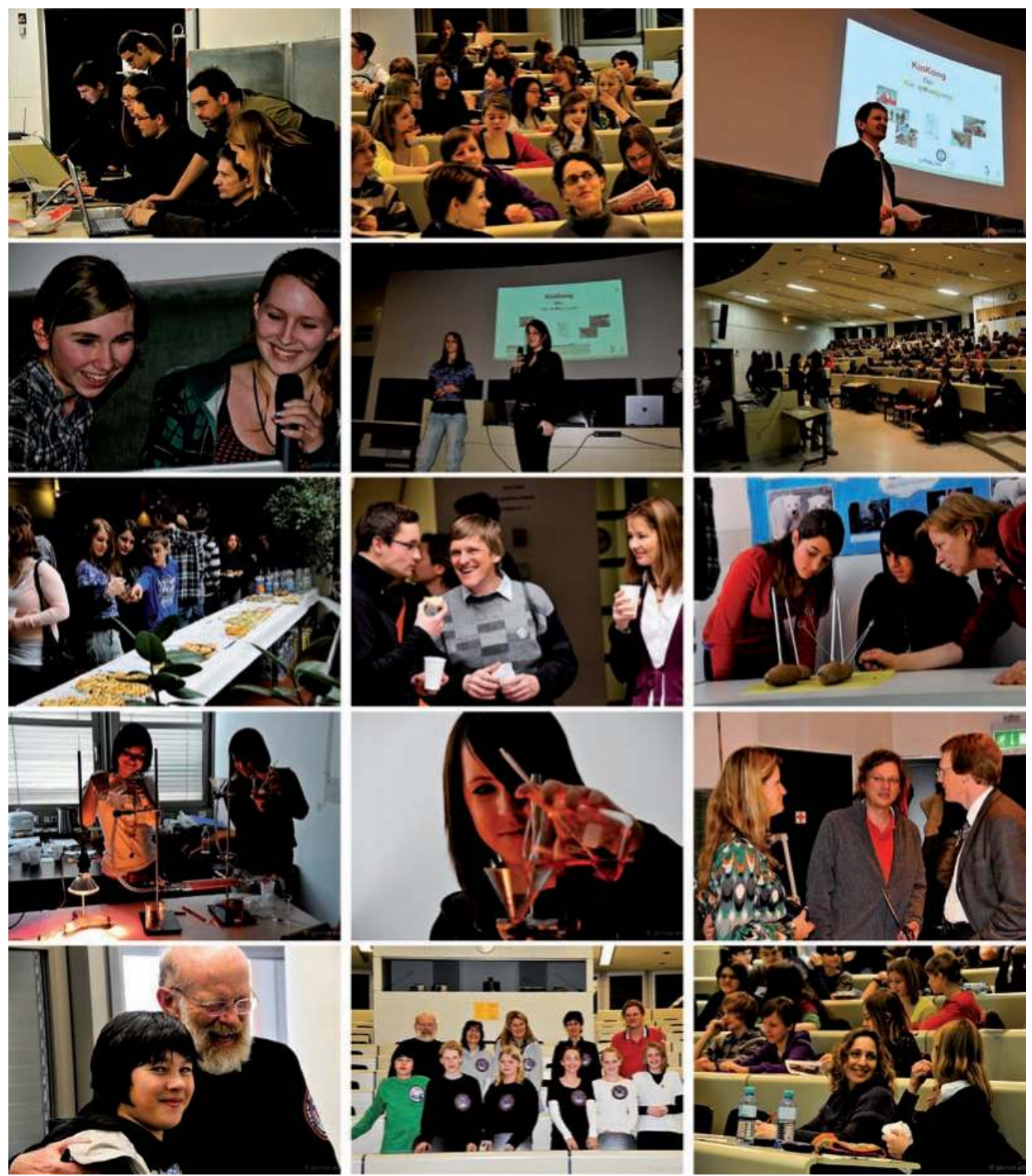

Fotos vom KinKong (Kinderkongress) am 20. Februar 2009 an der Universität Wien 
„Beinabe ein halbes Jahr dauerte unser Projekt. Schlussendlich fubren wir drei Tage nach Wien (zu unserem Bedauern mussten wir schon wieder auf den Schulunterricht verzichten), um unser Projekt am KinKong-Kongress vorzustellen. Die restliche Zeit in Wien bereitete uns viel Spaß.

Wir freuen uns schon auf unser nächstes Projek.t."

Johanna Lercher \& Nina Payr

„Der „KinKong“ in Wien war auch ein tolles Erlebnis. Die Präsentationen von den anderen Schulen waren auch interessant und man konnte viele nette Leute kennen lernen. "

Thomas Mader

„Vom 19. bis 21. Februar stellten wir unser Projekt in Wien am Institut für Ökologie vor. Es stieß dabei auf große Begeisterung und unsere Klasse war insgesamt sehr zufrieden mit diesem Projekt. Ich hoffe, dass wir nächstes Jahr ein ähnlich tolles Projekt starten können."

Fabian Mair

„Wien: Vorstellung der einzelnen Projekte leider eher langweilig; Stände teilweise nicht betreut oder uninformativ; technische Koordination mangelhaft, zuständige Personen unfähig, einen PC mit einem Beamer zu verbinden oder einen Dateinamen richtig zu lesen."

Andreas Maislinger

„Im Feber fuhr die ganze Klasse mit dem Zug nach Wien um unser Projekt, das wir ,Hilfe wir schmelzen" nannten, an der biologischen Uni beim KinKong vorzustellen. An unserem Stand konnte man die wichtigsten Informationen auf den Plakaten lesen. Einige Besucher spielten sogar unser selbst entwickeltes Spiel. Sogar die Spielfiguren stammten aus Eigenproduktion.

3 Schülerinnen durften am Tag der Politischen Bildung unser Projekt ein weiteres Mal vorstellen.

Mir hat das Projekt sehr gut gefallen, ich habe viel dabei gelernt. Wir hatten es bei unserem Projekttagen immer sehr lustig, auch wenn wir manchmal sehr unter Zeitdruck standen."

Claudia Müller

„Die Präsentationen begannen, und nach der Einleitung gleich wir. Hier überraschte uns die Inkompetenz. (Entschuldigung, aber so kam es wirklich herüber) sowie die unkooperative Einstellung der für den Präsentationssaal zuständigen Personen. Lautsprecher funktionierten nicht, der Computer war zu veraltet, um unseren 17 Sekunden Film abzuspielen, und obwobl wir einen brandneuen Laptop bereitgestellt bätten, stießen wir nur auf abwehrende Handbewegungen. Gekrönt wurde das Ganze dann noch durch die Einspielung der falschen Präsentation. Zwar hatten wir einen USB Stick mitgegeben mit der neueren und korrigierten Version, aus unerfindlichen Gründen entschied sich aber das Team dort, die alte Version mit mehreren angestrichenen Feblern einzuspielen. Das stellte sich natürlich erst bei der 3. oder 4. Powerpoint Folie heraus, und die Präsentation neu zu starten war uns dann doch zu blöd. Zusammengefasst: Projekt Hui, Wien pfui"

Alexander Sarg

„Als wir dann im Feber nach Wien fuhren war die Aufregung sehr groß. Obwobl ich es sehr interessant und spaßig fand, ging allerdings an der Uni Wien selbst so einiges schief. Stände waren teilweise 
nicht oder unsinnig betreut. Teilweise beschäftigten sich die Schüler nur selbst mit ibrem Stand und erklärten den anderen, die daneben standen nichts über ibr Projekt. Die Beiträge selbst waren sehr unterschiedlich und auch die Vorträge waren meiner Meinung nach von höchst unterschiedlichem Niveau. Der Höhepunkt war jedoch Folgendes: als wir dem, ich glaube man nennt ihn so, Medienbeauftragten unseren Laptop gaben, um unsere Präsentation auf den Computer der Uni zu laden, tat er dies, machte jedoch die falsche Präsentation auf!"

Stephanie Schmid

„Zum Abschluss des Projektes fubren wir gemeinsam für drei Tage nach Wien, um den KinKong ₹u besuchen. Wir bekamen einen Stand zugeteilt, bei dem wir Plakate aushängen, und unser Brettspiel und unsere Präsentation vorstellen konnten. Weiter hatten wir bei diesem Kongress die Möglichkeit, andere Stände zu besuchen und die Vorträge der anderen zu besuchen. Dadurch sammelten wir viele neue Informationen und lernten neue Leute kennen."

Madlen Schranz

„Die Tage in Wien mit dem Kongress waren sehr lustig und interessant, und wir hatten die Chance mit verschiedenen Wissenschaftlern und Polarforschern zu sprechen und einen Einblick in dessen Arbeit zu bekommen."

Eva Schwärzler

\subsection{Students on Ice: Expedition 2008 \\ Michaela Lurger, BRG Lilienfeld}

Edacational Expeditions to Antarctica and the Anctic

Am Beginn stand eine fächerübergreifende Projektarbeit im Rahmen von BIPOLAR. Unsere Arbeitsgruppe wurde ins Wissenschaftsministerium eingeladen. Ohne große Hoffnungen bewarb ich mich für den Hauptpreis, eine Teilnahme an der Students on Ice Expedition,

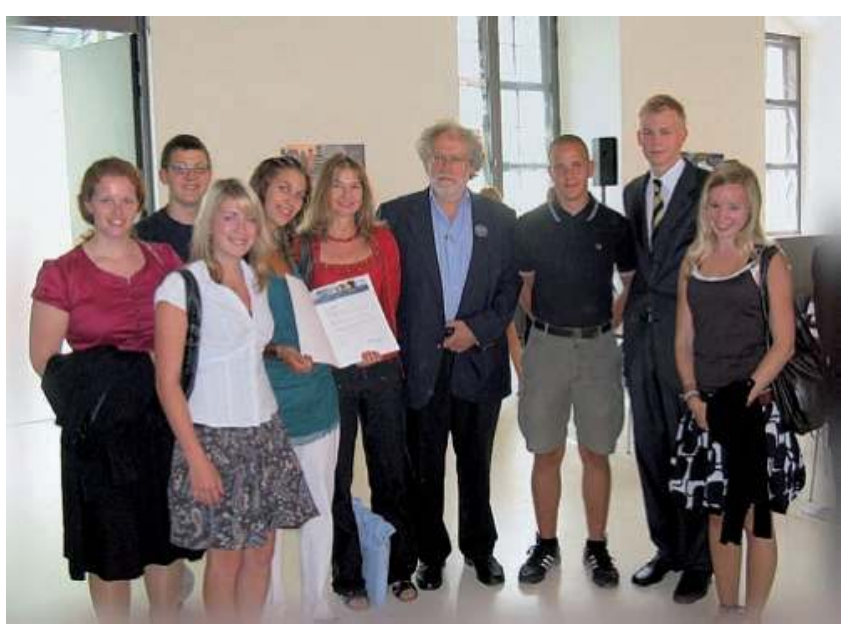
den ich überraschenderweise auch gewann. Der Preis, der die Schiffsreise in die Arktis beinhaltete, wurde von James N. Pritzker aus Chicago, USA, gestiftet. Der Flug von Österreich nach Kanada wurde von Wissenschaftsminister Dr. Johannes Hahn finanziert.

Anfang August startete ich meine Reise nach Ottawa, wo sich am Unicampus Studenten

Überreichung des Sparkling Science Preises 
und Schüler aus aller Welt mit Wissenschaftlern und Experten auf diese Expedition vorbereiteten. Nach einem kurzen Wochenende flogen wir gemeinsam nach Iqaluit, einem Dorf der Inuits, wo wir unser russisches Schiff bestiegen. Die nächsten zwei Wochen sollten mein Leben verändern. Unser tägliches Programm variierte je nach Wetterlage und Standort unseres Schiffes. An Bord gehörten Vorlesungen über den arktischen Lebensraum und Workshops wie zum Beispiel der Umgang mit GPS etc. zu unseren Aktivitäten. Den Hauptteil unserer Reise verbrachten wir jedoch an Land oder im Wasser. Mit Zodiaks fuhren wir entweder durchs Eis, auf der Suche nach Robben oder Walen oder in die Nähe von Klippen, die als Vogelbrutplätze dienten. Weiters unternahmen wir auch etliche Gletscherwanderungen, um dort Eisproben für weitere Untersuchungen zu entnehmen oder wir hielten uns in ehemaligen Walfängerorten oder in Standgegenden auf, um eine Runde in $4^{\circ} \mathrm{C}$ kaltem Wasser zu schwimmen. Der Kontakt zu den arktischen Ureinwohnern,
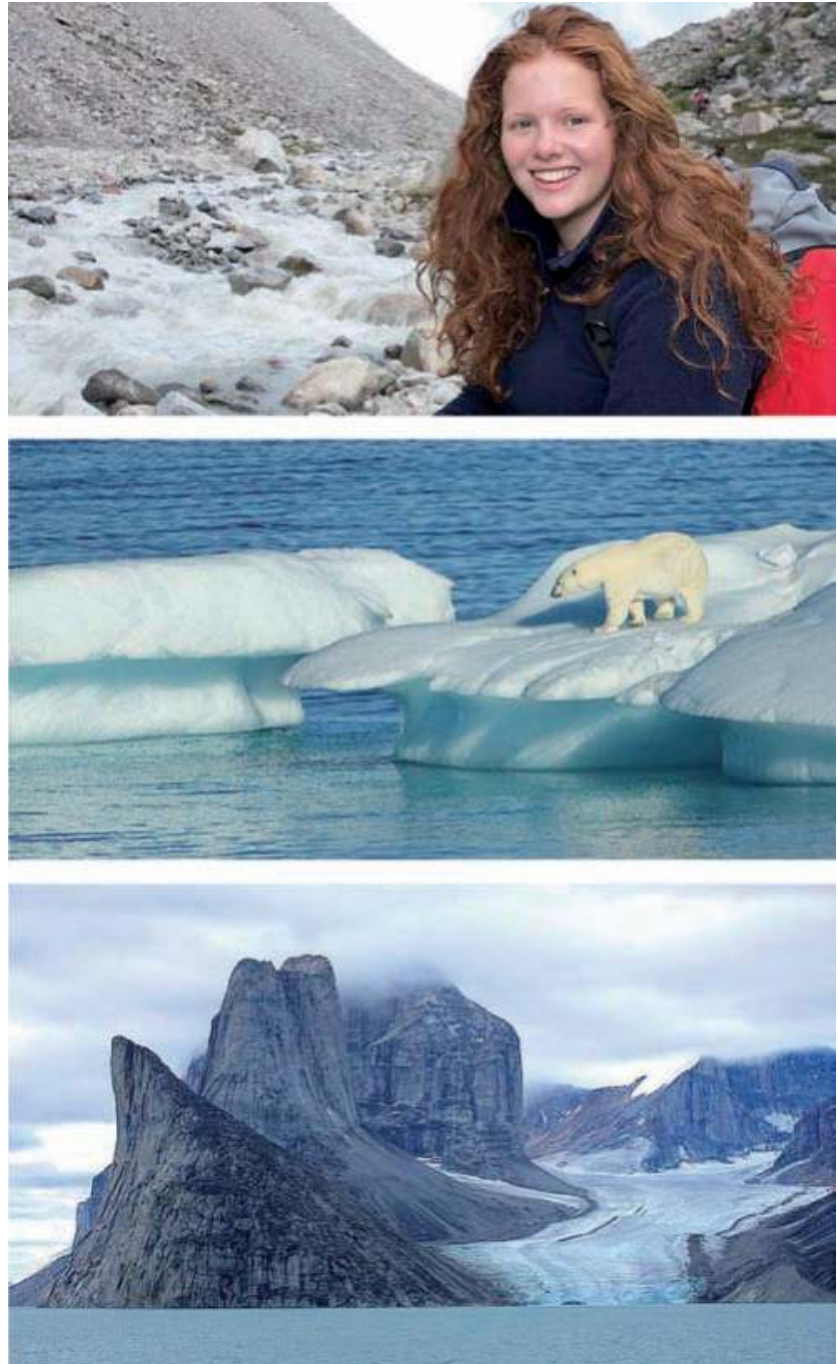

Impressionen aus der Arktis den Inuits, wurde auf der einen Seite durch eine Vielzahl von Schülern, die mit uns an Bord waren und auch durch einige Besuche in Inuitdörfern, wo wir öfters ein Barbecue mit ihnen veranstalteten, möglich. Erlebnisse wie das hautnahe Beobachten von unzähligen Walen, Robben oder Eisbären, beim Mittagessen rohen Fisch, Walfleisch und Karibu serviert zu bekommen, mitten in der Nacht aufgeweckt zu werden um Polarlichter zu sehen oder sich in einer anfangs völlig fremden, internationalen Gruppe integriert zu wissen, haben diesen Sommer auf eine ganz 

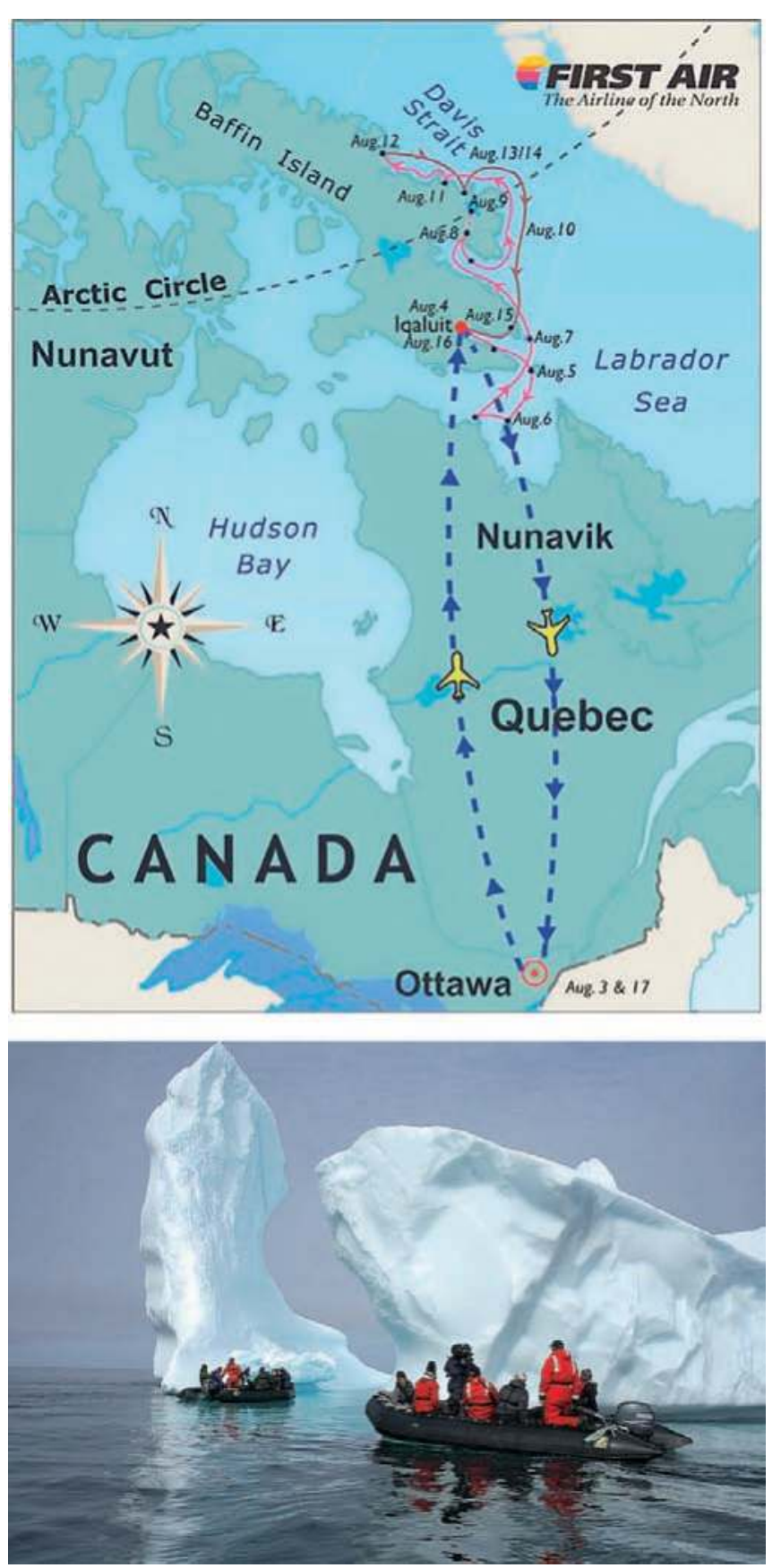

einzigartige Weise geprägt. Von dieser Reise durfte ich ein völlig neues Umweltverständnis, praktisches Wissen, traumhafte Eindrücke, sehr gute und enge Freunde und einen Berufswunsch mitnehmen.

Route der 'Students on Ice' Expedition im Sommer 2008

Exkursion mit dem Schlauchboot 


\subsection{Internationale Vernetzung - „Wir sind Expedition“ Birgit Sattler, Universität Innsbruck}

BiPolar ging im Laufe der Zeit über die Grenzen hinaus.

Ein großer Ansporn für Kinder und Jugendliche ist sicher, wenn sie in einer laufenden Studie involviert werden und mitgestalten können. Wenn diese Studie zudem international ausgerichtet und noch dazu an einem völlig exotischen Ort stattfindet, ist der Anreiz bestimmt noch größer.

Der wissenschaftliche Teil des Projektteams besteht durchgehend aus Personen, die - wie bereits erwähnt - selbst die weitergegebenen Ergebnisse erhoben haben an Lebensräumen, die nur wenige Menschen betreten können. Dieser Umstand schürte bei den SchülerInnen auch das Interesse an mehr bzw. an der Person, welche hinter einer Expedition steht. In diesem Fall bestand nun plötzlich für die SchülerInnen die Möglichkeit, selbst bei einer Antarktisexpedition involviert zu werden. Zwar bedauerlicherweise oder zum Glück nicht als Expeditionsmitglied, aber den Kindern kam bald eine sehr wichtige Rolle zuteil.

Die TAWANI Antarctic Expedition 2008 (http://expedition.tawanifoundation.com), deren Teilnehmer aus 4 Nationen bestand (USA, Russland, Neuseeland sowie Österreich) reiste im Winter 2008 in das Queen Maud Land, um von dort aus Extremstandorte zu untersuchen. Da auch diese Studie sich der Mikrobiologie in Eis-Ökosystemen widmete und ebenso wie das Projekt BiPolar unter dem Aspekt des Klimawandels stand, war der Kontext zu den SchülerInnen sofort gegeben.

Ein Großteil der Forschungsexpeditionen in Polargebiete hatte einen pädagogischen Kontext, nicht zuletzt wegen dem Internationalen Polarjahr, in welchem die nächste Generation auf allen Ebenen stark eingebunden wurde. Somit kooperierte auch diese Expedition mit 2 Lehrpersonen aus Illinois, USA, welche direkt vom Eis ihre persönlichen Erfahrungen und die neuesten Erkenntnisse oder Beobachtungen an die SchülerInnen einer Junior High School und einem College per E-Mail und Satellitentelefon weitergaben.

Die Hauptschüler aus Zirl wurden somit in die Expedition mit eingebunden - bereits vor der Abreise wurden sie in das Forschungsgebiet eingeführt und ansatzweise zu kleinen Eisexperten trainiert.

\section{Die SchülerInnen entwerfen ein Expeditionslogo}

Die erste große Herausforderung war ein ausgeschriebener Wettbewerb zwischen ihnen und Klassen der Junior High School in Illinois, USA, um ein Expeditionslogo zu gestalten. Unter großem Zeitdruck erstellten die Hauptschüler unter bester Betreuung, die weit über die in BiPolar involvierten Lehrpersonen hinausging, eine Reihe von äußerst professionellen Logos. Schlau gemacht, graphisch und optisch ansprechend, innovativ. Aus den über 20 Entwürfen wurde

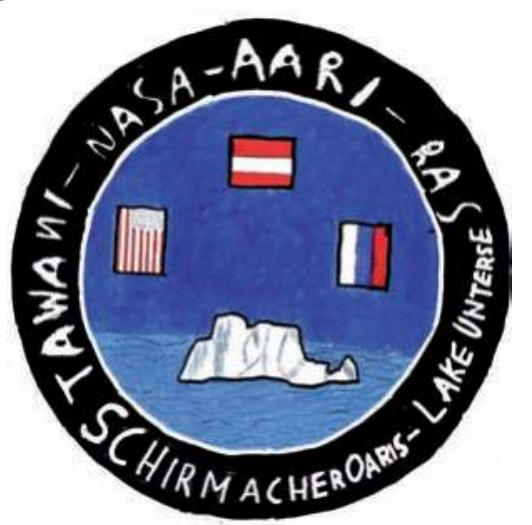


von einer größeren unabhängigen Gruppe ein Gewinnerlogo ausgewählt und in Windeseile gestickt.

Als die Schüler das fertige Emblem in der Hand hielten, hatten sie erstmals ein Produkt in der Hand, von dem sie wussten, dass es über eine längere Periode ihre Arbeit in die Welt hinaustragen und Beachtung erlangen würde. Es schuf bei den Schülern auch ein solidarisches Gefühl, es ging somit nicht nur einer von BiPolar auf Expedition, sondern sie wurden auf eine spezielle Art mitgenommen.

Auch bei den Expeditionsteilnehmern erzeugte dieses Abzeichen eine Verbundenheit zu den Kindern zuhause und zudem wurde es von den russischen Kollegen der Station Novolazarevskaya sehr gelobt. Als kleine Nebenbemerkung wurde ihr eigenes Logo mit Kopfschütteln bedacht, welches mit Hilfe eines bezahlten Designers erstellt wurde und die Russen daher gerne mit unserem getauscht hätten.

Der pädagogische Aspekt der Expedition richtete sich in diesem Fall beidseitig von den SchülerInnen an uns Wissenschaftler und umgekehrt, die uns vor meiner Abreise eine Liste an Fragen und Arbeitsaufträgen mitgegeben haben. Dieser Fragebogen hat uns Kopfzerbrechen bereitet, denn das Interesse der SchülerInnen richtete sich neben dem Alltag einer Expedition sehr auf unser Forschungsgebiet.

Die mitgeschickten Fragen reichten von „Wie tief muss man ins Eis bohren?“ oder „Wie schmeckt das Wasser?“, „Wie lange kann man seine Hand in das Wasser halten?“ „Welche Temperatur hat das Wasser im See?" bis hin zu Fragen über Bakterien - wieviele Arten von Bakterien wir finden würden und ob sie wohl sterben würden?

Diese Fragen haben über 10 Wissenschafter während einer isolierten Zeit auf dem Eis daran erinnert, dass dieses ungewöhnliche Habitat, an welchem wir unsere Zelte aufgeschlagen haben, auch Kinder fesseln kann.

„Antarktis calling“"

Die Forschungsreise zum permanent eisbedeckten Lake Untersee im Queen Maud Land der Antarktis ist noch wenig erforscht und gab uns jeden Tag neue Rätsel auf. Diese Fragen konnten wir mit den SchülerInnen einmal wöchentlich zu einer festgesetzten Zeit über ein Gespräch über Satellitentelefon teilen. Da das Expeditionsteam äußerst divers aus mehreren Disziplinen zusammengestellt war, konnten die SchülerInnen je nach Bedarf ihrer Fragestellung sich an verschiedene Experten widmen (z.B. Hydrologen, Mikrobiologen, Botaniker, Geologen), dies jedoch auf Englisch, dankenswerter Weise durch den Einsatz der Englischlehrerin der Hauptschule.

Der telefonische Kontakt zu den Kindern über tausende Kilometer hinweg hatte zudem eine bemerkenswerte Wirkung auf das Team auf dem Eis, welches völlig isoliert in dem Zeltcamp lebte. Diese Gespräche brachten für das gesamte Team fast schon ein Gefühl an Heimat, der regelmäßige Termin wurde zum Highlight der Woche und die kniffligen Fragen der Kinder wurden bereitwillig erklärt. Die Telefonverbindung wurde oft zum Abenteuer, denn oft machte Sturm das Gespräch fast unmöglich, doch die Kinder blieben hartnäckig und verloren auch nach mehrmaligen Ausfällen, wie es bei Satellitengesprächen üblich ist, nicht ihr Interesse. Meist erfuhren die Kinder als Erste von unseren neuen Entdeckungen auf der anderen Halbkugel. 
Diese Konversationen wurden von ihrem Klassenvorstand Markus Freiberger wie folgt beschrieben und auch digital aufgezeichnet.

„Die Kinder sollten via Satellitentelefon mit internationalen Wissenschaftern Kontakt aufnehmen, und zwar - ein besonderes Privileg - während des Vormittagsunterrichtes. Dass dies wieder ein Höhepunkt für die nunmehrigen Zweitklassler war, versteht sich von selbst. Das Faszinierende war dabei, wie die Telefonate, die ich auf meinem einfachen Handy speicherte, auf das Interesse der anderen Schüler stieß. Sie wollten das hören und auch darüber Bescheid wissen wie vierzehnjährige Teenies zuhören können. Wie ein Nasa-Forscher über den Lautsprecher des Handys spricht, wenn er von ausweichendem Gas an einem hyperalkalischen Süßwassersee in der Antarktis berichtet, war erstaunlich und beeindruckend - für alle Beteiligten. In diesen Augenblicken machte Forschung tatsächlich Schule. Die Schüler waren live dabei, als Chris McKay von Lebewesen berichtete, von denen man ihr Alter nur ahnen konnte und die zuvor dort niemand gesehen hatte. Mit dem Satz „Vielleicht finden wir solche Lebewesen in zwanzig oder dreißig Jahren am Mars und vielleicht ist einer von euch dabei“, verabschiedete er sich am Satellitentelefon und ließ eine Gruppe Kinder mit offenem Mund ein paar tausend Kilometer entfernt in einer Klasse zurück, wo ein Handy auf einem Tisch lag."

Sparkling Science hat einen äußerst positiven Effekt auf die internationalen Kollegen hinterlassen, einige von ihnen melden sich hier zu Wort:

\section{Poles of Mars \\ Michael C. Storrie-Lombardi ${ }^{1}$, Kinohi Institute, Inc. Pasadena, California U.S.A.}

Sparkling Science: From Lake Untersee, Antarctica, to the Icy

The polar and alpine snow, glaciers, and lake ice caps of planet Earth are home to rich microbial communities including photosynthetic organisms who significantly affect our world's food supply. Life capable of actually living inside of ice always seemed unlikely due to inhospitable living conditions. However, a decade of research in Antarctica, the Arctic, and the Alps has shown that Earth's cryosphere contains rich microbial food webs, so highly sensitive to environmental change that they can warn humanity of planetary-scale stress. But since life needs liquid water, how could life exist in

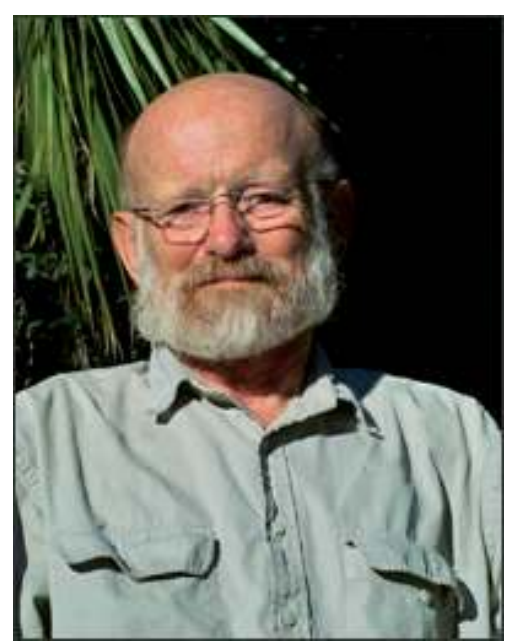

Michael C. Storrie-Lombardi

1 Dr. Storrie-Lombardi's interest in microbial life in extreme environments has led him to work in war zone surgical units in Congo and Vietnam, nuclear submarines and saturation dive stations deep beneath the ocean surface, the ancient rock deserts of the Pilbara outback in Northwestern Australia, and the alkaline lakes of the Sierra Nevada Mountains, eastern Washington State, and Antarctica. He is currently a participating scientist on the European Space Agency's ExoMars PanCam team. 


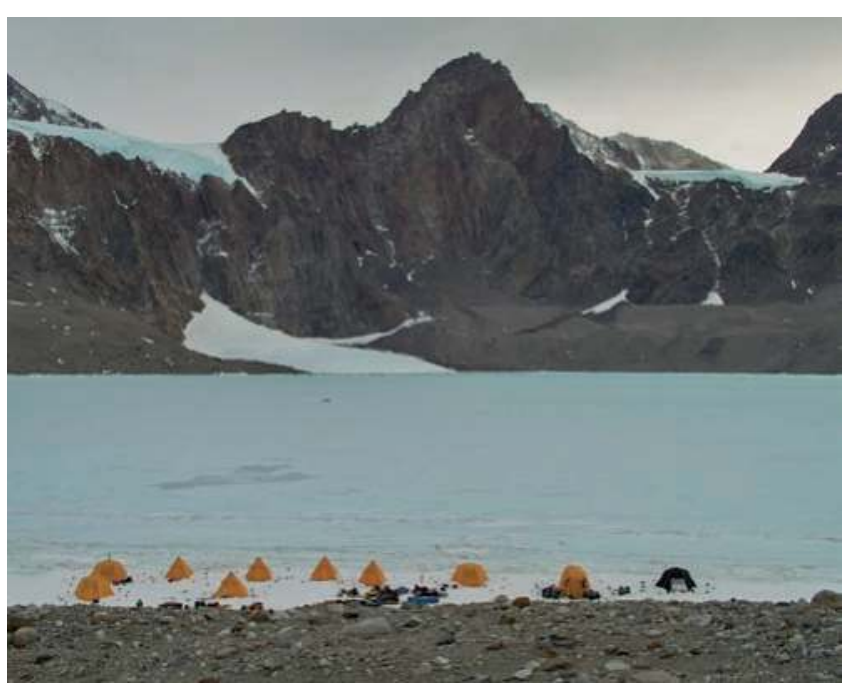

The camp site of the Tawani 2008 expedition to Lake Untersee in Dronning Maud Land, Antarctica. The radio contact with Sparkling Science children in Innsbruck was made via satellites that dipped bebind the mountain peaks.

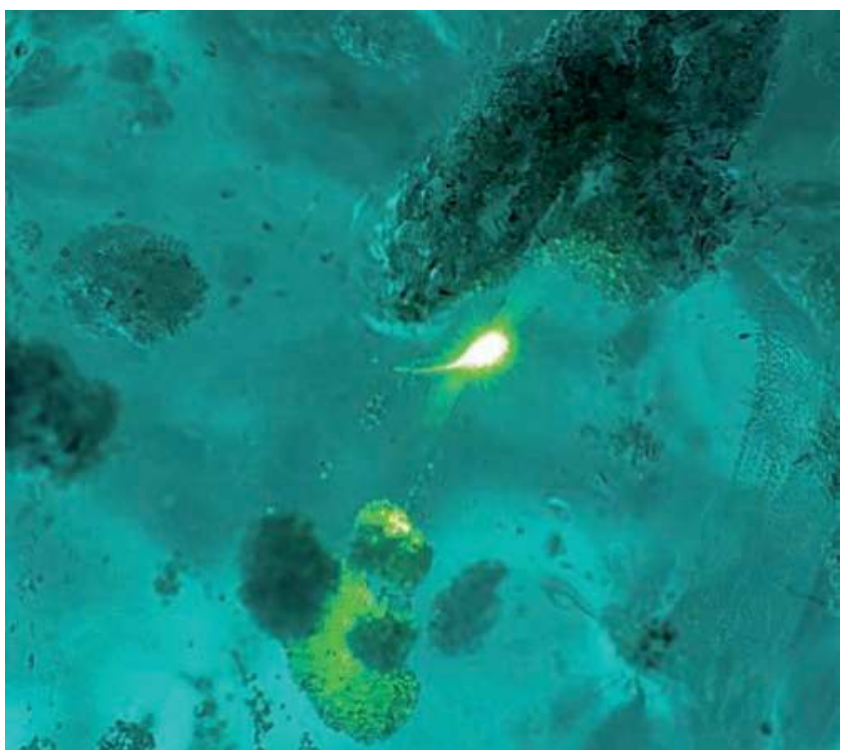

A green laser illuminates a dust particle embedded in the ice cap of Lake Untersee. Photosynthetic pigments in microbial life living in melt water around the dust send back a yellow fluorescence signature. the frozen wastelands covering our planet's polar and alpine regions? And, if life is abundant in these icy worlds, is there a way we could search for similar life forms inhabiting other planets in our Solar System or planets in neighbouring star systems?

To investigate these questions, a handful of scientists and teachers from Austria, the United States, Russia, and New Zealand journeyed to the Dry Valleys of Schirmacher Oasis and then on to the rarely visited Lake Untersee in Dronning Maud Land, Antarctica during the Tawani Expedition of October and November of 2008.

Lake Untersee is the largest freshwater lake of interior Antarctica. Situated at 71'20' S, $13^{\circ} 30^{\prime} \mathrm{E}$, the $10^{2} \mathrm{~km}$ lake arose from a melt-water pond during the Holocene and has been ice-covered for almost 10,000 years. With a maximum depth of about 180 meters, this alkaline lake is home to a previously unexplored, unique ecosystem. Life starts in the lake when microorganisms riding the winds attached to organic-rich dust land on the ice. Energy from the sun is absorbed by the dark dust, melts the underlying ice, and the mixture of dust and living microorganisms sinks into lake's ice cap. The liquid melt water covers the dust and soon freezes, but solar photons continue to pass easily through the clear ice, warm the dust, and 
produce a thin layer of liquid water around the grains and the bacteria. The combination of dust, water, photosynthetic microbes, and sunlight produce a tiny ecosystem, a miniature Earth, acquiring both liquid water and useable energy from the sun. Photosynthetic microorganisms use the sunlight, water, and the nitrogen and carbon dioxide from air bubbles to make food critical to all other life forms on our planet.

Recent advances in laser and camera technology made it possible for us to obtain laserinduced fluorescence emission (L.I.F.E.) images of the life inhabiting these miniature worlds (Figure 2). In other words, we can shine a green or red laser pointer at these organisms and watch them glow. We learned at Lake Untersee that we could do this crawling around in the wind and cold with a hand-held camera and laser. We now know we can do it from an airplane flying over frozen lakes and rivers in Alaska, and one day we hope to do the same thing from a satellite circling the Earth, Mars, or a planet in another star system.

While we were slipping and sliding around on the ice of Untersee taking photographs with our tiny laser, I assumed what we were doing in Antarctica was of interest only to a handful of us who design equipment and do research to monitor climate change on Earth or search for life on other planets. But all of that changed one evening in November of 2008. My colleague in the effort to build a laser L.I.F.E. imaging system is Dr. Birgit Sattler of the Ecology Institute at the University of Innsbruck. I knew she was recording sounds of our expedition and accumulating photographs to take back to children in Innsbruck and Vienna. But I was pleasantly surprised when she said that the next morning the children were going to be calling us on our radio phone from a classroom in Innsbruck, some 10,000 kilometers from Untersee. The call came in on schedule the next morning. In spite of repeated interruptions as the satellite relay disappeared momentarily behind the gargoyle peaks of the mountains surrounding Untersee, the children asked multiple questions about our research at the lake. I later would visit those same children in Innsbruck to hear their thoughts about the radio contact, but at the time, answering questions while standing on a frozen lake in spite of language, space, and electronic barriers, I was struck by three things.

First, the questions were incredibly well-researched. They were not random pleasant inquiries. The children had heard about the mysterious gas bubbles we had seen coming up from the lake and wanted to know their origin. They knew the growing season was very short and wanted to know how rapidly photosynthesis began to work in the springtime. They knew we were going to start diving beneath the ice soon and wanted to know what we expected to see. They wanted to know how we kept warm and if we were able to sleep with sunlight shinning on us twenty four hours a day. And they wanted to know if we really ate 5000 calories of chocolate a day as they had heard.

Second, I was struck by their enthusiasm. What I had thought was esoteric work of interest to only a few colleagues, was clearly important to them. I imagined that I could hear in the voices of some of the children, both girls and boys, the hope that one day they, too, might come to a magnificent place such as Untersee, perhaps on another planet, and ask their own questions of nature.

Third, I recall being aware of the impact that the radiophone interview had on me and on the other scientists in our team. When you are thousands of kilometers removed civilization, when your world of societal contact is limited to less than a dozen human beings, even the most devoted scientist has moments of wondering what all the work and all the effort 
is about. Will it lead anywhere? Will someone else pick up the banner when our time on the planet has come to an end? The voices on the other end of the radio answered all of those questions. The earnestness, the interest, the eagerness, and the curiosity of these children translated across all languages and multiple generations. The enthusiasm and thoughtfulness of these youngsters told us in no uncertain terms that the banner would be passed to a new generation. A phone call originally designed to contribute to the education of a small group of children had contributed profoundly to a small group of Antarctic scientists a long way from home.
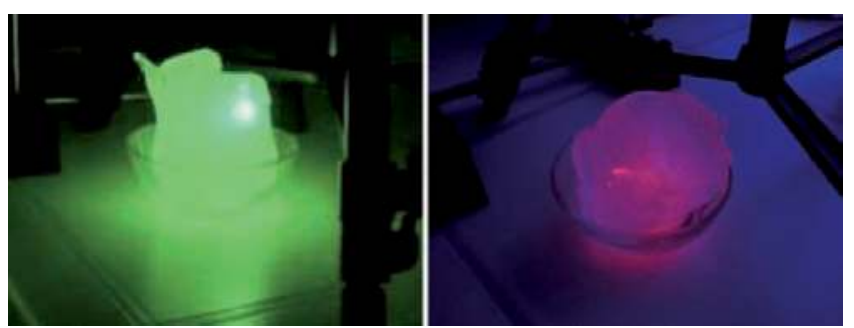

Artificial ice cores inhabited by living microbes and illuminated by red and green laser light as part of a Sparkling Science classroom exercise in Innsbruck in the spring of 2009.
Some months following my return from Antarctica, I received an invitation to present a lecture and a "laser life-in-ice show" in Innsbruck to many of the children who spoke with us over the radio while we were working at Lake Untersee.

I also joined several of them as they presented their own work at the Kinder Kongress in Vienna in the spring of 2009. On both occasions I was once again struck by the enthusiasm, the carefully crafted questions, and the novel ways the children proposed to investigate life in the ice. While it is certainly logical for all of us as parents to pursue efforts such as Sparkling Science for the benefits it will bring to our children, I must admit there is also another reason to support the work. I have been a physician and scientist for more than four decades. I have witnessed the effect a single Sparkling Science effort made on multiple members of my own generation, scientists normally separated by geography, language and politics, but drawn together for a few moments as they shared a common language of science with children far, far away. The impact of Sparkling Science has been as profound as any educational activity I have seen while working in America, Europe, Africa, Vietnam, and Australia. I consider the Sparkling Science effort a remarkable credit to Austrian science and education, and hope it sparks similar projects around the world. As for my own small contact with the Innsbruck children in the project, I fully expect to see at least one of them broadcasting from somewhere near the North Pole of Mars, sometime in the middle of the $21^{\text {st }}$ century. 
"One of the most important things we did on this expedition was the public information and educational programs. We had the ability to use satellite phones and the internet to share our polar science research and show the sense of adventure and discovery."
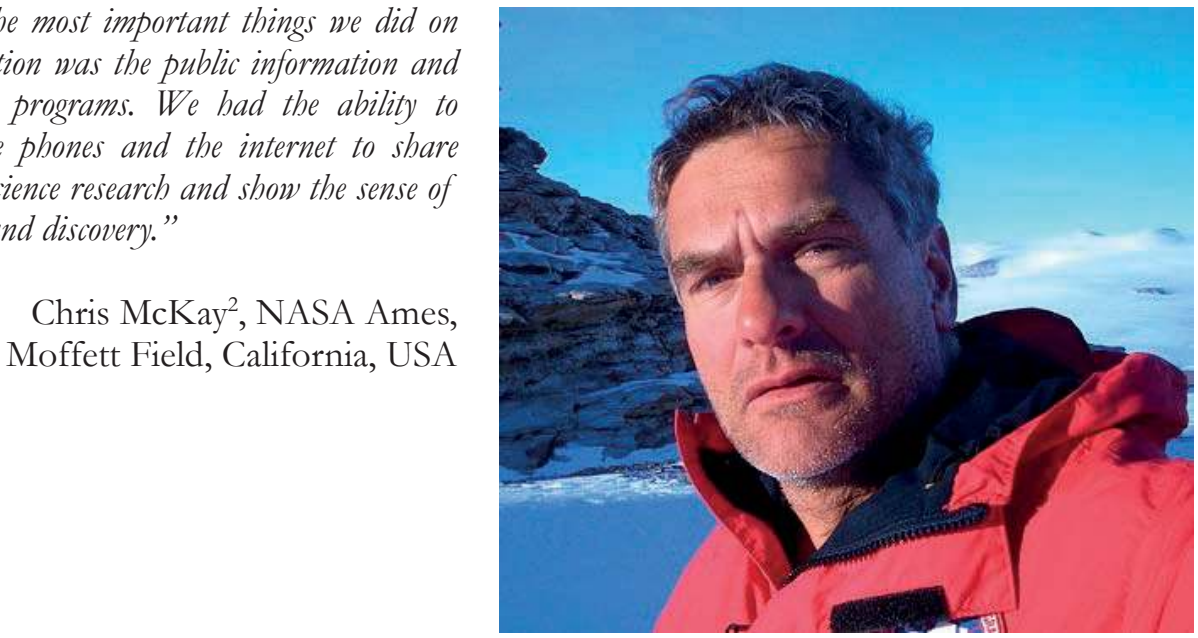

Chris McKay

2 Planetary Scientist with the Space Science Division of NASA Ames. Ph.D. in AstroGeophysics and research scientist with the NASA Ames Research Center. His current research focuses on the evolution of the solar system and the origin of life. He is also actively involved in planning for future Mars missions including human exploration. 


\section{Einbindung in andere internationale Programme}

Das Projekt BiPolar konnte auch in anderen pädagogischen Programmen der USA seine Anhänger finden. So plant die non-profit Organisation Wings World Quest (ww.wingsworldquest.org), welche den Sitz in New York hat und Frauen in Pionierrollen fördert, das Projekt in ihrem entstehenden Programm „DISCOVER!“‘ aufzunehmen, wobei die BiPolarKinder eine Vorreiterrolle spielen und amerikanische Kinder motivieren sollten, die Chance für derartige Projekte zu nutzen bzw. ihr Interesse für Wissenschaft zu fördern.

Einen weiteren Schritt in Richtung USA haben die

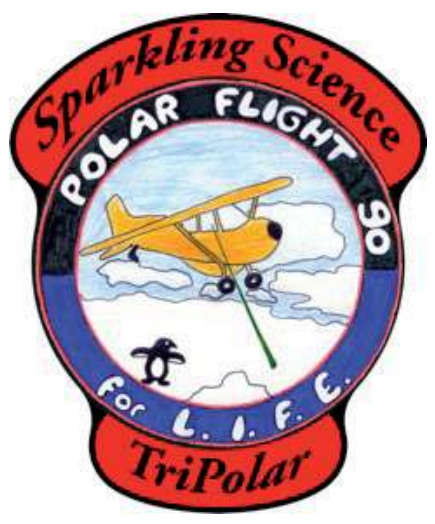
Kinder bereits getan, indem sie durch das Expeditionslogo bereits einen bestimmten Bekanntheitsgrad erreicht haben. So wurden sie von Art Mortvedt, einem Buschpiloten aus Alaska, aufgefordert, für sein einzigartiges Projekt, alleine mit einer einmotorigen Cessna zum Nordpol zu fliegen, ein Logo zu erstellen (www.polarflight90.com). Dieses Projekt wurde bereits mit dem Charles Lindbergh-Award ausgezeichnet und tourt bis zum tatsächlichen Abflug im April 2012 durch ganz Amerika mit Vorträgen zur Expedition und der daran geknüpften Wissenschaft. Das Flugzeug mit dem Namen Polar Pumpkin wird für den Flug mit einem Logo einer Schülerin der damals 2. Klasse Hauptschule in Zirl geschmückt. 


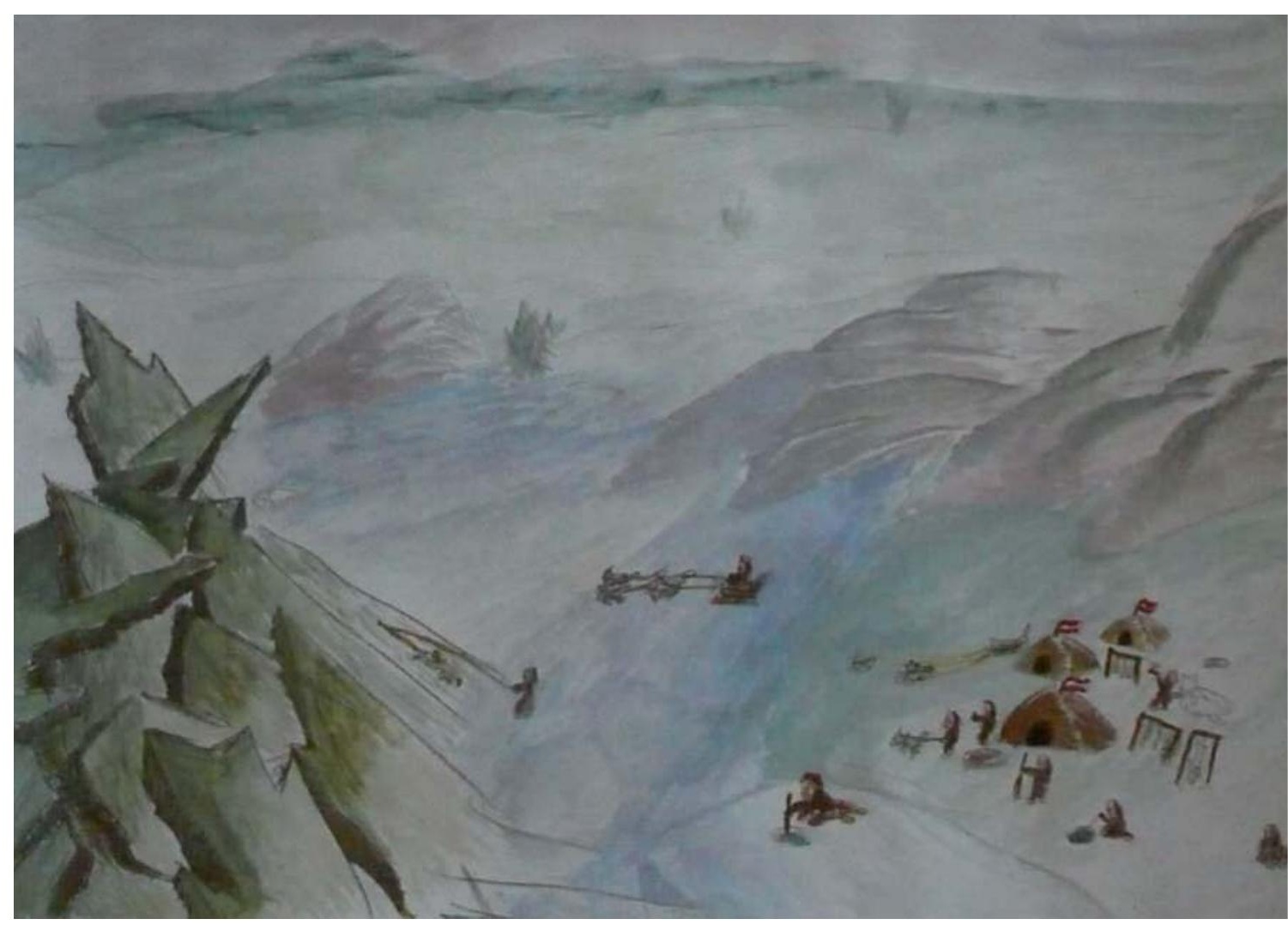

Bild: Julian Prochaska, 2008

\section{Kapitel 5}

Wissenschaft geht an die Öffentlichkeit (Science goes public) 



\section{Wissenschaft geht an die Öffentlichkeit (Science goes public)}

„Die Inhalte und Ergebnisse von BiPolar haben nicht nur den beteiligten Schulen und WissenschafterInnen viel gebracht, sondern sollen auch außenstehenden Interessierten zur Verfügung stehen. Deshalb wurden in den zwei Projektjahren auch zablreiche öffentliche Aktivitäten gesetzt, die im folgenden Kapitel besprochen werden."

\subsection{Eisbär, Pinguin \& Co. Bettina Girschick, Elfriede Schmid, Elisabeth Fuchs BRG 6 Marchettigasse Wien}

Mitten in den heißen Sommerferien, am 28.7.2008, brach die „Polardelegation“ der Marchettigasse in voller Ausrüstung für sämtliche Polar-Experimente in den Tiergarten Schönbrunn auf.

Dieser Tag stand nämlich unter dem Motto „Eisbär, Pinguin und Co.“ und reihte sich mit den Projektpräsentationen in die Veranstaltungsreihe ein, die im Rahmen der Artenschutztage in Schönbrunn abgehalten wurde. Gemeinsam mit VertreterInnen der ZAMG und der Universität Wien präsentierten wir das BiPolar-Projekt. Die SchülerInnen und Schüler führten ihre spannenden Experimente über Polartiere vor, aus denen deutlich wurde, wie außerordentlich die Anpassungsleistungen der Tiere an die extremen Lebensbedingungen in der Arktis sind. TeilnehmerInnen und ZuseherInnen versetzte manche Erkenntnis in Staunen.
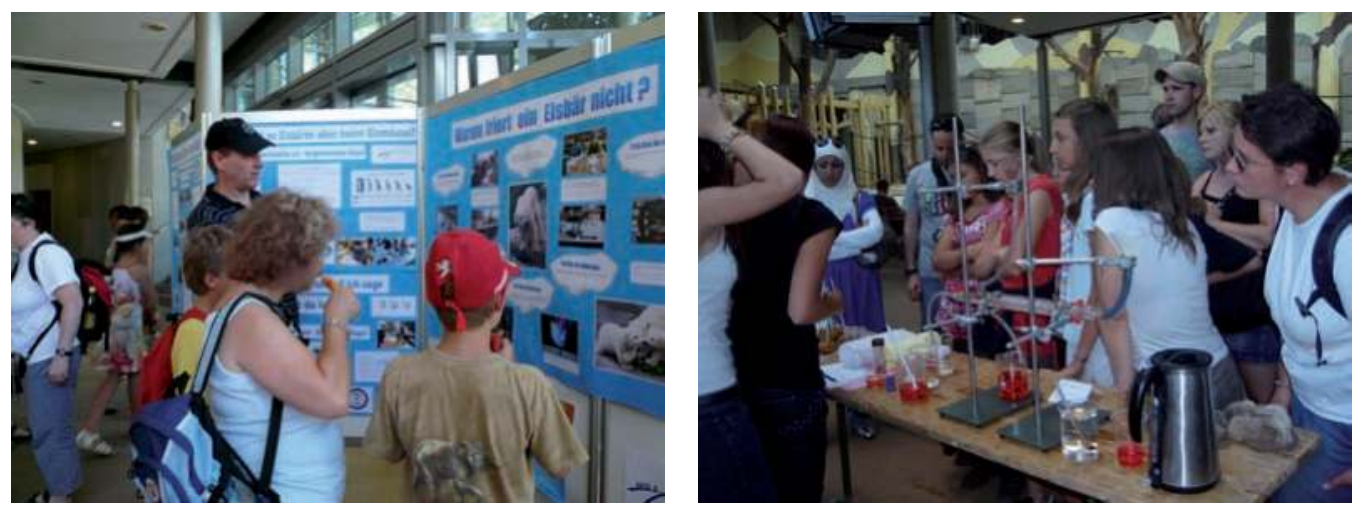

Bei der Prjektpräsentation in Schönbrunn 
Wie kam es dazu? Den Auftakt zum BiPolar Projekt stellte ein Bericht über die Expeditionen in den arktischen Lebensraum dar, der uns von Frau Mag. Michaela Panzenböck sehr anschaulich und lebendig präsentiert wurde, so dass der Funke der Begeisterung übersprang: besonders von den Eisbären und Moschusochsen und den aufregenden Schilderungen über die Begegnungen mit diesen Tieren waren die SchülerInnen angetan.
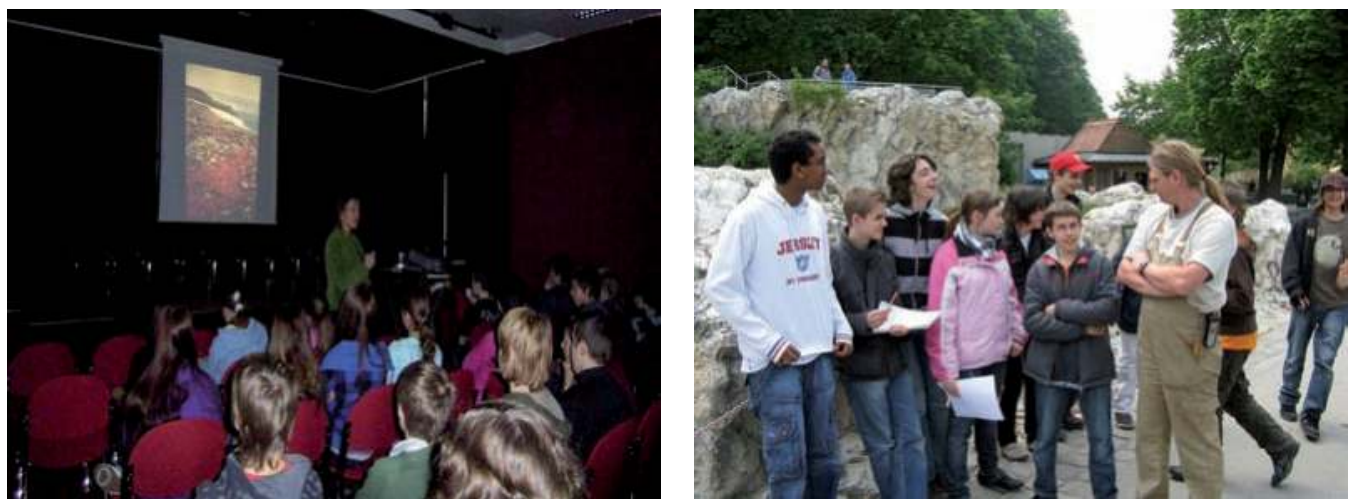

Vorstellung der arktischen Lebensräume

Daher auch die Entscheidung, uns mit den Polartieren schwerpunktmäßig zu beschäftigen. An einem fächerübergreifenden Projekttag (Biologie, Chemie, Physik und Geografie) erforschten wir die Tricks der Polartiere in der eisigen Kälte zu überleben.

Im Zuge der intensiven Beschäftigung mit dem Thema stellte sich uns die Frage, wie es denn die Polartiere schaffen, in unserem Klima - gemäßigte Zone - im Tiergarten Schönbrunn zu überleben? Sogleich machten wir uns auf in den Tiergarten, wo wir nach einer Führung die Pfleger der Polartiere ausführlich interviewten.
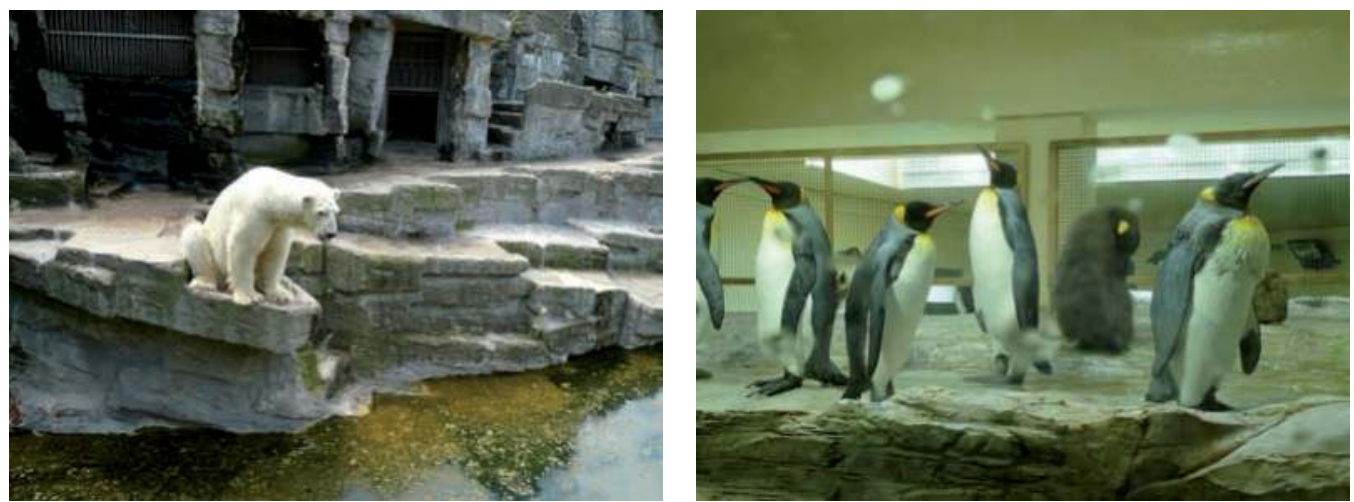

Eisbär als Repräsentat für die arktische Fauna, Pinguine für die Antarktis. Beide Bilder augenommen in Schönbrunn. 
So erfuhren wir beispielsweise, dass die Königspinguine im Zoo auch im Süßwasser gut leben können, dass die Raumtemperatur aber auch im Sommer 15 Grad Celsius nicht übersteigen darf, hier ist Leben im gekühlten Raum erforderlich! Eisbären bekommen aufgrund des Fehlens von Wildtieren in der Nahrung täglich einen Viertel Liter Lebertran zur Erhaltung der dicken Fettschicht und zur Deckung des Vitamin A- und D-Bedarfs.

Die Ergebnisse unserer Tierpfleger-Befragung sind in der unten stehenden vergleichenden Tabelle zusammengefasst.

Aus dieser Kooperation mit dem Tiergarten Schönbrunn ergab sich dann die Möglichkeit, an den Artenschutztagen teilzunehmen. Die SchülerInnen freuten sich darüber, ihr Wissen, das sie sich in den begleitenden fächerübergreifenden Workshops in der Schule angeeignet hatten, auch in der Öffentlichkeit präsentieren zu dürfen. Als Standort für die Demonstration unserer Experimente wurde uns das Elefantenhaus zur Verfügung gestellt. Die Wissenschafterinnen und Wissenschafter der Universität Wien und der ZAMG projizierten beeindruckende Bilder von ihren Expeditionen, wir stellten die Plakate über unsere BiPolar-Aktivitäten in der Schule aus und bauten unsere Modellversuche auf. Folgenden Fragen waren wir auf den Grund gegangen:

„Wie schützen sich Tiere vor der Kälte?“

„Warum gibt es Eisbären, aber keine Eismäuse?“

„Warum friert ein Eisbär nicht?“

Als spannende Antworten erhielten wir folgende:

Beispielsweise schützen die Pinguine ihre Füße auf eine ganz spezielle Art vor dem antarktischen Eis: Ihre Blutgefäße in den Füßen und Flossen arbeiten im Gegenstromprinzip. Das kalte, von den Füßen kommende Blut in den Venen wird von dem warmen Blut, das sich auf dem Weg vom Herzen befindet, erwärmt - die wärmenden Arterien sind nämlich unmittelbar neben den Venen platziert! Diesen Trick konnten wir in einem Modellversuch mittels Dimroth-Kühler anschaulich demonstrieren.
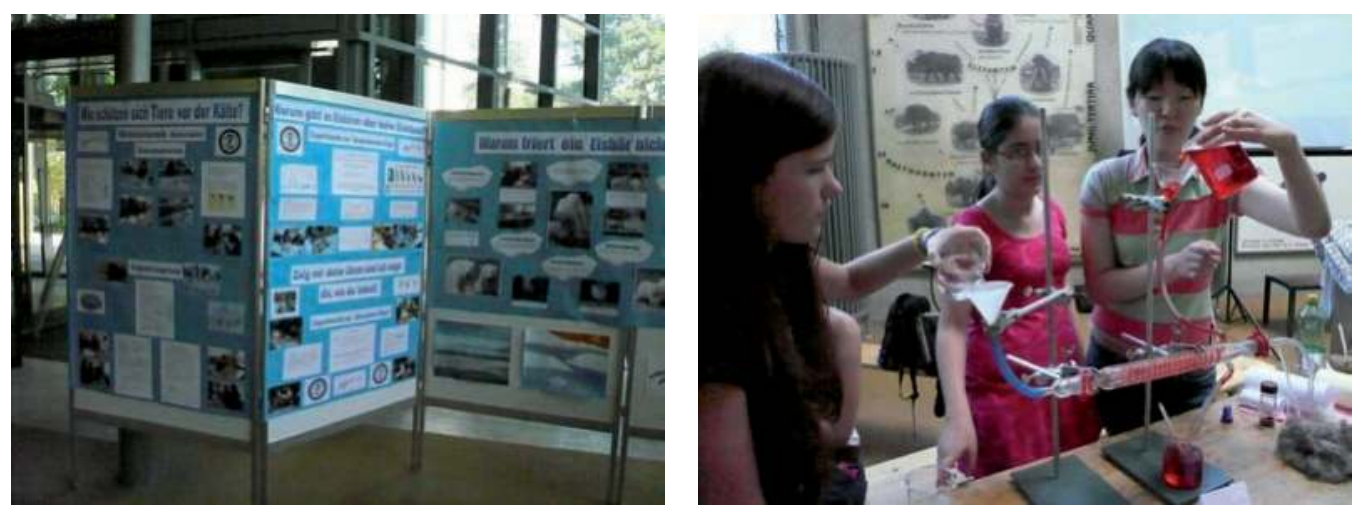

Präsentationen zum Thema Kälteanpassung 
Eine weitere wichtige Anpassungsleistung von Polartieren ist die Ausbildung einer Wärme isolierenden Körperbedeckung, wie einer dicken Fettschicht, eines dichten Fells oder eines dichten Gefieders.

Aber auch in ihrem Sozialverhalten passen sich Tiere an die extreme Kälte und den starken Wind an: Pinguine und Moschusochsen beispielsweise rücken nahe zusammen. Bei den Moschusochsen zeigen dann die wenig behaarten Häupter in das Innere des Kreises, während das dicht behaarte Hinterteil nach außen der Kälte zugewandt wird. Pinguine dagegen wechseln regelmäßig ihre Position von außen nach innen und umgekehrt.

Dass auch Größe und Leibesfülle wirksam gegen die Kälte eingesetzt werden können, veranschaulichten die SchülerInnen mit dem „Kartoffelversuch“: gekochte große Kartoffeln
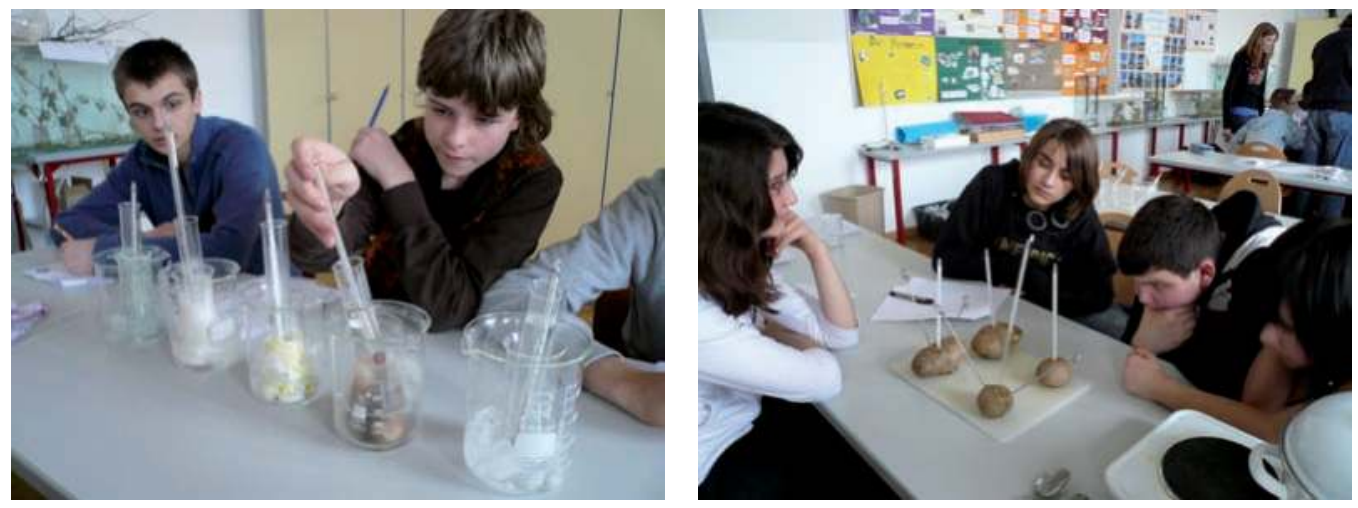

Versuche zum Wärmeverlust: Bergmannsche und Allensche Regel.

(„Eisbären“) kühlen langsamer aus als kleine („Eismäuse“).

Damit wäre die Bergmannsche Klimaregel bewiesen: verwandte Wirbeltiere in kälteren Regionen fallen generell größer aus als jene in warmen Regionen und sie kühlen daher langsamer ab.

Zusätzlich verwendeten wir Metallspieße („Ohren“) in den heißen Kartoffeln und konnten dadurch die Allensche Klimaregel demonstrieren. Diese geht davon aus, dass über die Köperanhänge wie Schwanz und Ohren leichter Wärme abgegeben wird. Daher sind die Körperanhänge - wie Ohren oder Schwanz - bei Polartieren kleiner als bei Vertretern derselben Gattung in warmen Regionen.

Auch der Eisbär hat verschiedene Tricks um sich vor Eiseskälte zu schützen. Im einfachen Modellversuch mit zwei Dosen, einer schwarz und einer weiß bemalten, die mit einer Lampe bestrahlt wurden, zeigten unsere SchülerInnen, dass seine schwarze Haut (schwarz bemalte Dose mit Temperaturfühler) im Vergleich zu heller Haut (weiße Dose mit Temperaturfühler) die Sonnenstrahlung und auch die diffuse Strahlung besser absorbieren kann.

Aber auch gegen die Abstrahlung der eingefangenen Wärme verfügt der Eisbär über einige Tricks: er besitzt nicht nur eine dicke Fettschicht, sondern auch ein weißes Fell mit vielen 

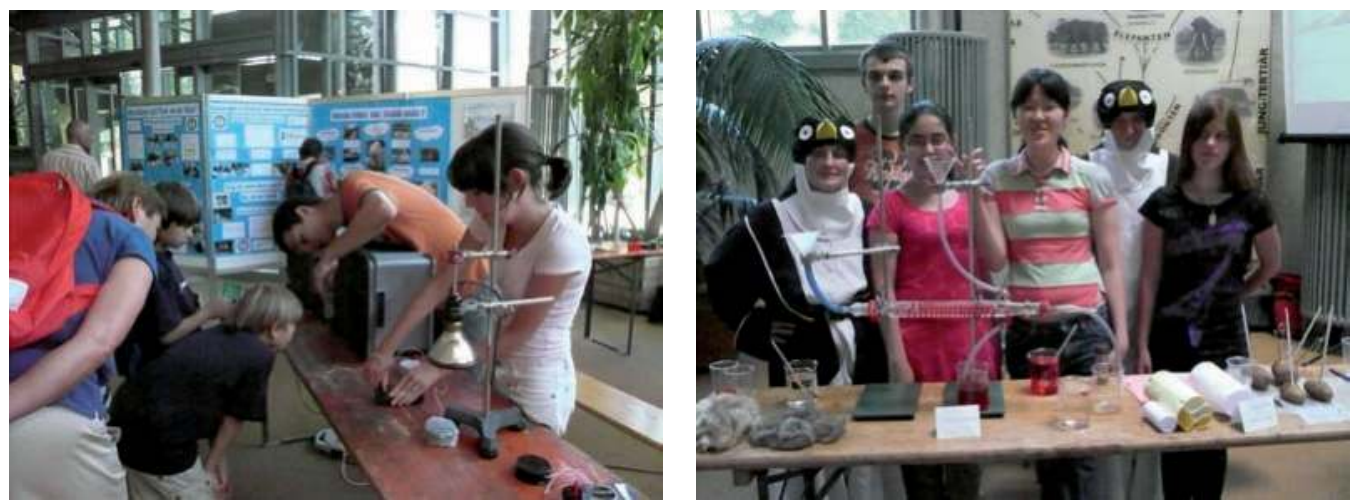

Versuche zur Absorption von Wärmestrablung und zur aktiven Isolierung durch Gegenstrom-Wärmetausch.

eingeschlossenen Luftpolstern, die isolierend wirken. Weiters sind die Haare des Eisbären hohl, sie fangen das Licht auf und leiten es - wie durch einen Lichtleiter - direkt auf die Haut. Das Eisbärenfell leuchtet sogar im UV-Licht, das ließ sich mit einer UV-Lampe wunderbar demonstrieren! Das Fell nimmt die energiereiche UV-Strahlung auf, ein Teil davon wird in Wärme umgewandelt, die restliche Energie wird dann wieder im sichtbaren Bereich abgestrahlt. Insgesamt gibt der Eisbär so wenig Wärme ab, dass man ihn mit einer Infrarotkamera nicht sehen kann. Er ist wirklich ein „,cooles“ Tier!

Das Interesse der Besucher an den Präsentationen im Elefantenhaus war trotz sommerlicher Hitze groß. Auch die Zoodirektorin Frau Dr. Dagmar Schratter und die „Pinguine“ aus der Antarktis statteten unserem Stand einen Besuch ab.

Bei einem Podiumsinterview wurde unser BiPolar-Team zum Projekt „Eisbär, Pinguin und Co." und zu den Aktivitäten im Tiergarten befragt. Die SchülerInnen schienen die Arbeit sehr genossen zu haben.

Es hat ihnen sichtlich Spaß gemacht, ihr Wissen auch außerhalb der Schule weiterzugeben, noch dazu unter der inspirierenden Anleitung und Mitarbeit von Wissenschafterinnen und Wissenschaftern:

„Es war echt toll, den Leuten etwas über die Polargebiete beizubringen. Die meisten waren erstaunt über unser großes Wissen." (Yasmin). „Es war ein schöner, erfolgreicher Tag und es hat sehr viel Spaß gemacht!" (Sina)

„Ein heißer, aber echt cooler Ferientag mit coolen Experimenten!" (Melanie)

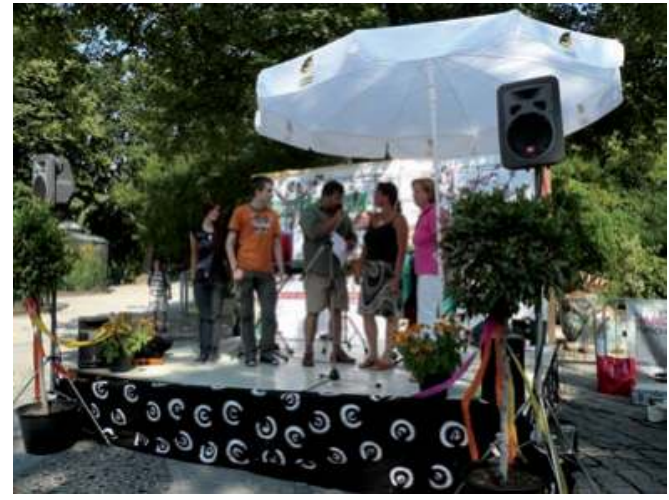

Podiumsinterview,Eisbär, Pinguin und Co.". 


\subsection{Brettspiel BiPolar}

\section{Silke Jung, BRG Sillgasse Innsbruck}

Neben der Homepage entwarfen wir ein Brettspiel zu den Themen Geschichte, Biologie und Geographie der Arktis/Antarktis mit Fragen- und Antwortkärtchen.

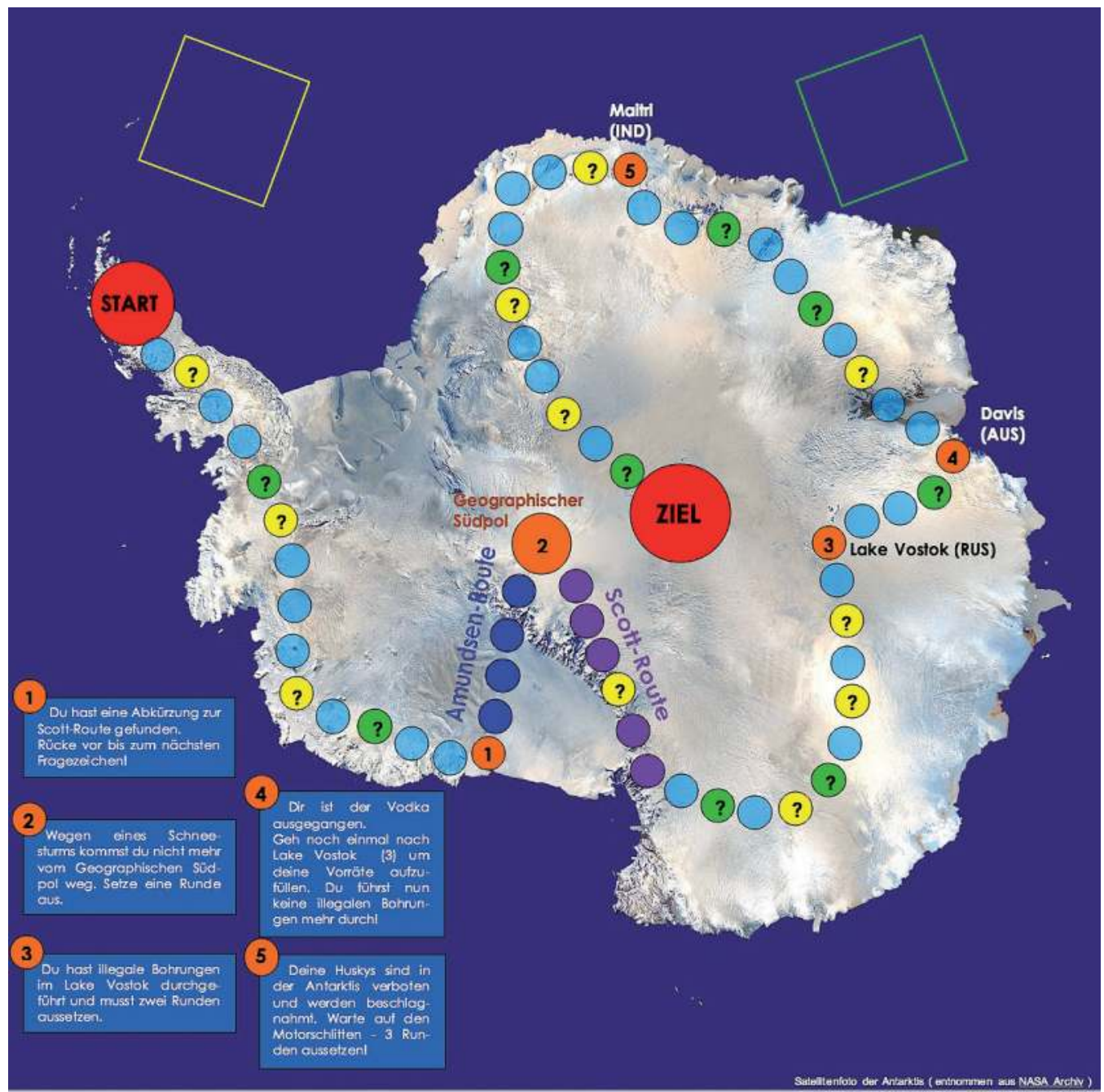

Spielfeld zum Brettspiel BiPolar 
Spielerzahl: 2-6

\section{Spielregeln}

Alter: 13-99

\section{Inhalt:}

Spielplan; 6 Figuren; Fragekärtchen leicht (gelb, 33 Stück) und schwer (grün, 38 Stück) für Geographie, Geschichte und Biologie, 1 Würfel

\section{Spielverlauf:}

Alle Spielfiguren werden auf dem START-Feld platziert. Der jüngste Spieler beginnt, würfelt und setzt seine Figur auf das entsprechende Spielfeld.

Kommt ein Spieler auf ein Fragenfeld (mit einem „?“ gekennzeichnet), muss er eine Frage beantworten, die der Spieler zu seiner Linken von dem entsprechend gefärbten Fragenstapel aufnimmt und samt den Antwortmöglichkeiten vorliest.

Alternativ dazu kann entweder nur mit gelben (leichten) oder nur mit grünen (schwierigen) Fragen gespielt werden.

Wird die Frage richtig beantwortet, geht er normal weiter, beantwortet er sie falsch, bleibt er an Ort und Stelle und muss in der nächsten Runde erneut eine Frage beantworten.

Kommt ein Spieler auf ein Aktionsfeld (mit einer Ziffer gekennzeichnet), muss er die am linken unteren Rand des Spielfeldes angeführte Aktion durchführen.

\section{Ziel des Spiels:}

Der erste Spieler, der das Ziel erreicht, gewinnt.

\subsection{Gemeinsame BiPolar - Ausstellung von der ErLEBnis- schule Mödling und dem BG Bachgasse, Mödling Herfried Weiss, BRG Bachgasse Mödling Irene Wailzer, Erlebnisschule Mödling}

Unser Thema: Klimawandel - beobachtet auf der Pasterze / Großglockner und am Goldbergkees / Sonnblick

Angesprochen auf die Teilnahme am BiPolar-Projekt im Zuge des Internationalen Polarjahres 2008/2009 hatten die Schülerinnen und Schüler der 5a des BG Bachgasse in Mödling folgende Idee: „Wir wollen die Veränderungen durch die Erderwärmung am „ewigen Eis“ bei uns in Österreich beobachten“ „Und wir drehen einen Film darüber!“

Geplant wurde also ein Dokumentarfilmprojekt über den Rückgang der Gletscher durch Klimaerwärmung am Beispiel Pasterze (Großglockner). In Zusammenarbeit mit Wissenschaftlern der ZAMG (Zentralanstalt für Meteorologie und Geodynamik) und der Universität Wien, Biozentrum war die Klasse mit ihren Lehrern gemeinsam 4 Tage lang auf der 
Pasterze unterwegs, um dem Rückgang des Gletschereises auf den Grund zu gehen. Es entstanden dabei 4 Filme und mehrere Plakate mit den Forschungsergebnissen. Die Sekundaria der ErLEBnisschule Mödling untersuchte den Goldbergkees des Hohen Sonnblicks ebenfalls zusammen mit Wissenschaftlern der ZAMG Wien. Die SchülerInnen selbst waren Wissenschaftler und Forscher am Gletscher und lernten unter Anleitung verschiedene Messmethoden kennen. Auf der Uni Wien nahmen sie die Eisproben des Gletschers unter die Lupe. Der entstandene Film zeigt Eindrücke und Erlebnisse dieser Projektwoche.

Mit der Ausstellung im BG Bachgasse Mödling am 23. November 2009 wollten die SchülerInnen die Inhalte, die sie selbst erarbeitet haben, mit der Öffentlichkeit teilen - auch Versuche durften dabei nicht fehlen.

Die Ausstellung war 3 Tage lang am BG Bachgasse in Mödling zu sehen. Zur Eröffnung fanden sich prominente Gäste aus Wissenschaft und Politik: Dr. Celine Loibl, Koordinatorin der Sparkling Science Projekte im Wissenschaftsministerium; Fr. Stadträtin Verena Schwendemann in Vertretung des Hrn. Bürgermeisters; Fr. Direktor Barbara Ramos, ErLEBnisschule Mödling; Hr. Dir. Mag Reinhard Gogola, Dir und Gastgeber des BG Bachgasse, Mödling; VertreterInnen der Presse und aller Klassen der Schule so wie natürlich die Schüler und Schülerinnen der Klassen, die am Projekt teilnahmen.

Trotz nur 3 tägiger Dauer besuchten mehr als 15 Klassen die Ausstellung im Zuge des Unterrichts und fast die komplette Schule (derzeit rund 1000 Schülerinnen und Schüler) in den Pausen und ihrer Freizeit.

Für alle Beteiligten war es eine sehr gewinnbringende, interessante, schöne Zusammenarbeit Schule-Wissenschaft! Die dabei gewonnenen Eindrücke sind sicher jene, an die man sich auch nach der Schullaufbahn noch gerne zurück erinnert!
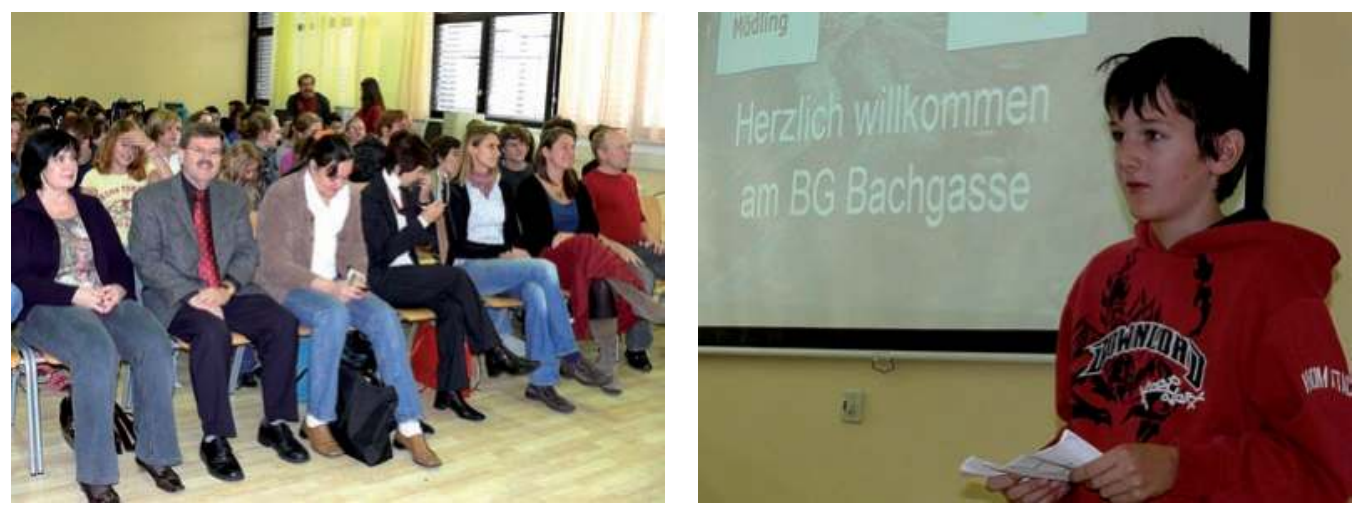

Bilder von der Eröffnung der Ausstellung am BG Bachgasse in Mödling 


\subsection{Pressespiegel Silvia Prock, Universität Innsbruck}

Sowohl in „West-“ als auch in „Ostösterreich“ wurden zahlreiche Berichte über das BiPolarprojekt veröffentlicht. Vor allem die Preise, die das Projekt erhielt, wie der Sparkling Science Schulpreis 2008 und der IMST Award 2009, erhöhten das Interesse der Medien an dem Projekt.

Im Folgenden gibt es eine Zusammenfassung der medialen Berichterstattung, die keinen Anspruch auf Vollständigkeit erhebt. Vor allem die zahlreichen online-Berichte im Internet wurden nur auszugsweise erfasst.

- Heißer Draht bis in die Antarktis, Kronenzeitung, 1. Dezember 2008, S 17

- 5000 Euro für Junge Polarforscher!, Kronenzeitung, 30. Juni 2008, S 12

- Tiroler Schüler-Expedition aus Zirl wurde ausgezeichnet, Bezirksblatt, Nr. 27, 2. Juli 2008, S 6

- Polar-Nachwuchs forschte eifrigst, Kronenzeitung, 5. Mai 2008, S 20

- $\quad$ Polarexpedition ins Kühtai, Tiroler Woche, 2. Mai 2008, S 15

- $\quad$ Expedition: Schülerin erforscht die Arktis, Kronenzeitung, 24. August 2008

- Wissenschaft ruft Schule, www.uibk.ac.at/ipoint, 27.Juni 2008

- Sparkling Science: Wissenschaft ruft Schule-Schule ruft Wissenschaft, technik im kopf, Verlagsbeilage der Wiener Zeitung, S 32

- $\quad$ Sparkling Science: Schule ruft Wissenschaft - Wissenschaft ruft Schule, bm.w_f, S 11

- Junge Polarforscher, ORF Tirol Heute, 30.4.2008

- Junge Polarforscher, ORF Radio Tirol

- $\quad$ Antarktis, bitte melden! TT, 5. Dezember 2008

- $\quad$ Eis, Schnee und warme Pinguinfüße, KiKu, 8.März 2009

- $\quad$ Schüler erforschen Eisbären und Gletscher, APA 20. Feber 2009

- $\quad$ AWARD für Zirler Schulprojekt, Bezirksblatt, 14. Oktober 2009

- Arktis und Antarktis - Terra incognita? Jahresbericht des Gymnasiums Adolf-PichlerPlatz, Juli 2009

- $\quad$ Forschungsprojekt wird ausgezeichnet, TT, 30. September 2009

- $\quad$ IMST-Award für das Projekt BiPolar, ipoint am 1. Oktober 2009

- Die Donaustadt räumt bei Schuloscar ab, Kurier, 1. Oktober 2009

- $\quad$ Auszeichnung für Jungforscher, Rundschau, 22. Oktober 2009

- Gletscherwoche der 4C Klasse, Info des Gymnasiums Kalksburg

- Symposium zum internationalen Polarjahr, ipoint am 22. Jänner 2008 
- $\quad$ Schul-Oscar geht nach Zirl, Krone, 19. Oktober 2009

- Polarforschung im Klassenzimmer, Kleiner Botschaften der Forschung, Presse, 25. August 2007

- $\quad$ Eis, Schnee und warme Pinguinfüße, Kurier 8.3.2009

- Junge Polarforscher, ORF Tirol Heute, 2008

- $\quad$ Forsch doch mal! SCIQ, S 12

- Jahresbericht Gymnasium Lilienfeld 2007/2008

- Jahresbericht Gymnasium Lilienfeld 2008/2009

Online (Auszüge)

- Vorstellung des Projektes durch die HS Zirl bei der „yo-tech“ - dem 7. Informationstags für eine technische Ausbildung für die Unter- und Oberstufe am 24. Juni 2008: http: / / set-career.eu/news-store/yo-tech-technik-im-kopf.html/

- Sparkling Science Homepage - http://www.sparklingscience.at/de/projects/212-bipolar/; http://www.sparklingscience.at/de/projects/238-ice-life/.

- IMST-Award - http://imst.uni-klu.ac.at/award/

- IMST-Award für das Projekt BiPolar - IMST-Award für das Projekt BiPolar-http:// imzoom.info/article.php/20090929085256188?query=IMST

- IMST Award für HS Zirl - http://content.tibs.at/index.php?menu=5\&con_id=28689 


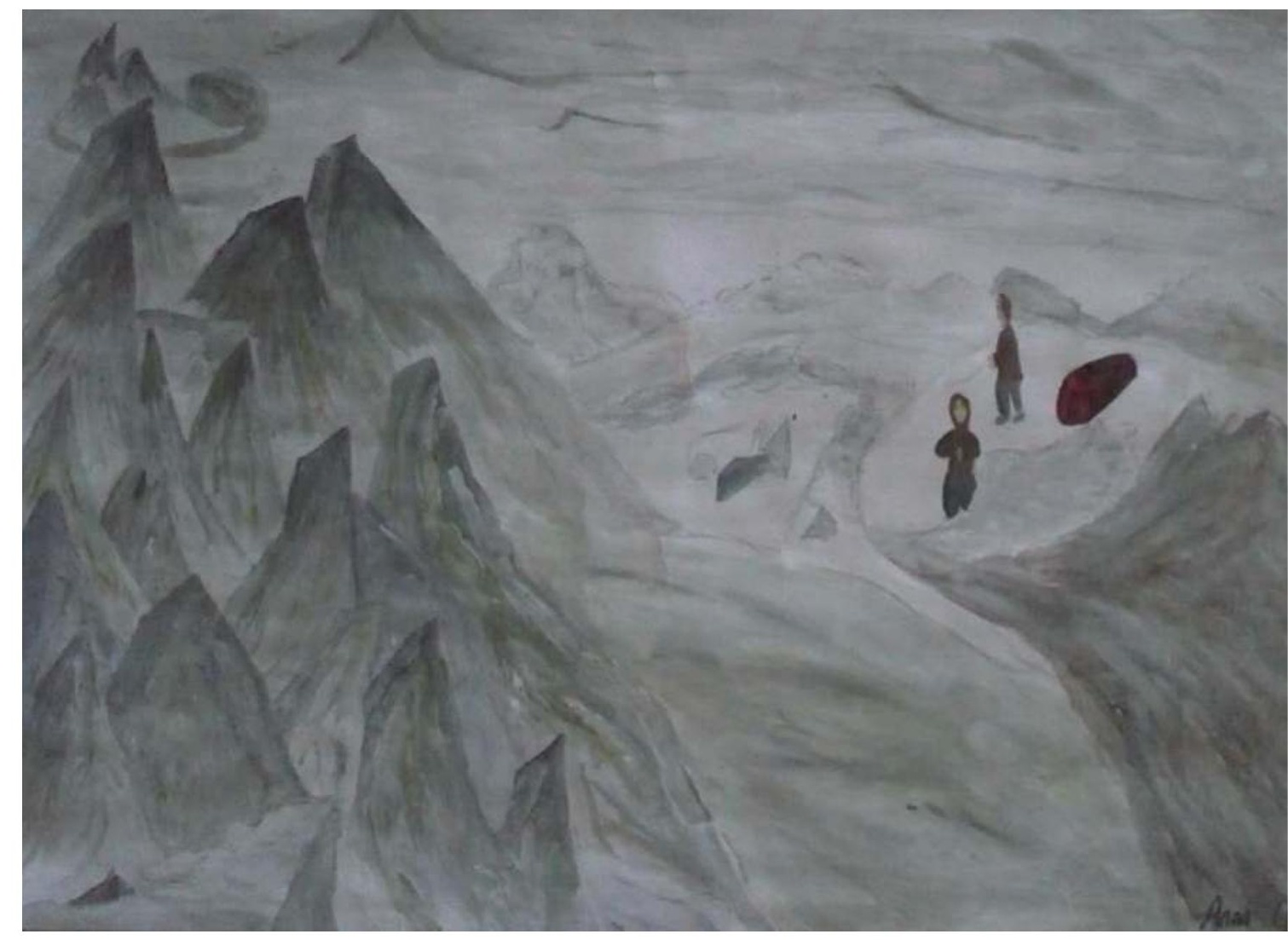

Bild: Anna Cho, 2008

\section{Kapitel 6}

\section{Erfahrungen und Nachhaltigkeit, Evaluation}





\section{Erfahrungen und Nachhaltigkeit, Evaluation}

\subsection{Erfahrung Wissenschaft Birgit Sattler, Universität Innsbruck}

Ohne die Bereitschaft und die Flexibilität der Lehrerinnen und Lehrer ist dieses Vorhaben unmöglich und zum Scheitern verurteilt. Der Mehraufwand war in vielen Bereichen beachtlich, allein, was den Organisationsaufwand für Lehrpersonen betrifft, wenn eine Schulklasse auswärts ist und die dabei verlorenen Stunden wieder aufgeholt werden müssen. In dieser Hinsicht ist der Bereich der Wissenschafterinnen und Wissenschafter glücklicherweise etwas freier in der Zeiteinteilung.
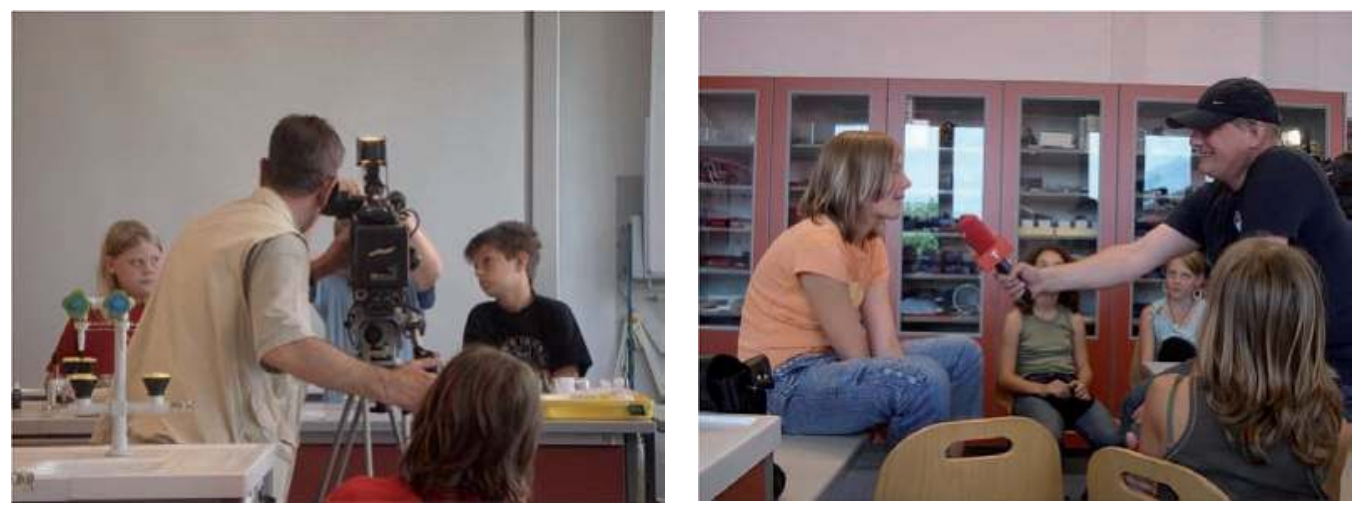

Präsenz in den Medien: Filmaufnahmen und Interviews in der Scbule.

Durch den Kontakt mit internationalen Forschungspartnern im Zuge der TAWANI Antarctic Expedition wurde auch den Erwachsenen bewusst, wie wichtig der pädagogische Inhalt der Forschung sein kann, oder zumindest der Versuch. Durch die Miteinbindung der SchülerInnen im Zuge der Erstellung eines Expeditionslogos wurde dadurch eine andere Identifikation mit der Sache erreicht. Die darauffolgenden Presseberichte und Auftritte im ORF (siehe Pressespiegel) avancierten somit zur erhofften Bestätigung für die Kinder. Auch der Respekt der Wissenschafter den Schülern und ihrer Fragen gegenüber geht in Richtung des „Ernst-Nehmens“.

Die Schüler des BRG Adolf Pichler-Platzes erstellten zum Beispiel ein Produkt aus ihren Recherchen, welches hoffentlich den Weg zum Verkauf finden wird. Das Spiel „Hilfe, wir schmelzen!“ ist ein außerordentlich klug durchdachtes Konzept mit Unmengen an Information. Sollten derartige Produkte und Ideen verstauben, dann mindert es natürlich die Motivation. Ist es jedoch etwas Nachhaltiges, von dem die Schüler auch nach Jahren sagen können, „das haben wir damals produziert und es wird immer noch verwendet“", dann ist ein Ziel erreicht. Der Wunsch für dieses Spiel ist nun, es in einer größeren Auflage auf Port 
Lockroy, einer britischen Station in der Antarktis, welche sich unter der Schirmherrschaft des United Kingdom Antarctic Heritage Trust in einem Geschenkeshop zu vertreiben.

Das Projekt hat nicht nur bei den Teilnehmern der TAWANI-Expedition Anerkennung erhalten (wo es auch auf der offiziellen Expeditions-DVD verankert ist), sondern auch bei anderen Organisationen, welche sich mit pädagogischen Elementen und Herstellung neuer Schnittstellen befassen. Die non-profit Organisation „Wings World Quest“" mit Sitz in New York, USA, hat es sich zum Ziel gemacht, Wissenschafterinnen mit Pionierleistungen zu fördern und auszuzeichnen. Das Programm Sparkling Science hat ihre Aufmerksamkeit erregt und somit wurde eine ähnliche Schiene auf ihrer Plattform erstellt, welche sich „DISCOVER!“ nennt. Die internationalen Preisträgerinnen sollen in diesem Programm ihre Forschung in gleicher Weise an SchülerInnen weitergeben (in Form eines Webauftrittes). Die Kinder von BiPolar sind bereits Bestandteil dieses Programmes und sollen auch andere Kinder motivieren.

Die SchülerInnen haben durch ihre profunde Frage, wie es in der Arktis und in der Antarktis so klingt, eine Geräusch-DVD initiiert. Mit einem mp3-Aufnahmegerät wurden verschiedene Geräusche auf dem Eis aufgenommen und zu einer DVD zusammengestellt. Erstmals wurde diese Sammlung auf dem KinKong in der Lauschbox vorgestellt. Die verbesserte Version davon „Sounds of Antarctica“ wurde bereits in Chicago im Field Museum vorgestellt und sogar vor einigen Tagen auf der besagten antarktischen Station verkauft.

Es geht aber nicht nur um verwertbare Ideen, sondern vor allem: was bleibt?

Was bringt diese Zusammenarbeit einem/einer WissenschafterIn? Vielleicht wird diese Frage irgendwann obsolet sein und es wird zur Bringschuld, Wissenschaft der nächsten Generation weiterzugeben und sie mit ihnen zu erarbeiten. Wir geben ihnen Probleme in die Hand, welche vielleicht erst diese Generation lösen kann. Diese Kontinuität gelingt aber nur, wenn die notwendige Biegsamkeit in den respektiven Institutionen gewährleistet werden kann und wenn es gelingt, „sich in der Mitte zu treffen“.

Die Arbeit mit Kindern bringt einem/einer WissenschafterIn neben den emotionalen Aspekten völlig neue Impulse, neue Perspektiven, andere Wertigkeiten der Fragestellungen. Aber wenn es gelingt, den Schülern Forschung aus unserer unmittelbaren Umgebung schmackhaft machen zu können, so wecken wir vielleicht auch eine Sensibilisierung dafür. Auf jeden Fall sollte die Neugierde seitens der SchülerInnen bleiben, und seitens der LehrerInnen und WissenschafterInnen die Bereitschaft, diese Abenteuer zu wagen. 


\subsection{Zwischenevalution}

\section{Silvia Prock, Universität Innsbruck}

Im März 2009 wurde von Silvia Prock von der Universität Innsbruck eine Zwischenevaluation des BiPolar-Projektes durchgeführt. Drei Fragebögen wurden entwickelt, die an die drei am Projekt beteiligten Gruppen - LehrerInnen, SchülerInnen und WissenschafterInnen angepasst waren und ausgesandt wurden. Die Fragebögen sind im Anhang einsehbar. Es wurden Fragen quantitativer und qualitativer Natur gestellt, wobei die qualitativen Fragen überwogen.

\section{Stichprobengröße}

Insgesamt konnten 51 Fragebögen ausgewertet werden. Absolut gesehen wurde der Großteil der Fragebögen (42) von SchülerInnen ausgefüllt, relativ gesehen waren aber die WissenschafterInnen (67\% aller Wissenschafterinnen) am eifrigsten, da insgesamt fünf WissenschafterInnen am Projekt beteiligt waren.

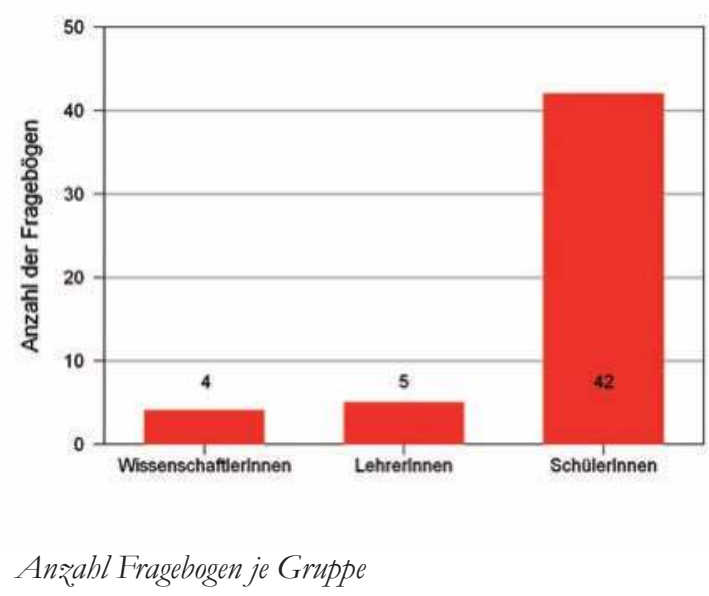

\section{Geschlecht}

Bei den WissenschafterInnen und den LehrerInnen antworteten gleich viele Männer wie Frauen, bei den SchülerInnen deutlich mehr Schüler als Schülerinnen.

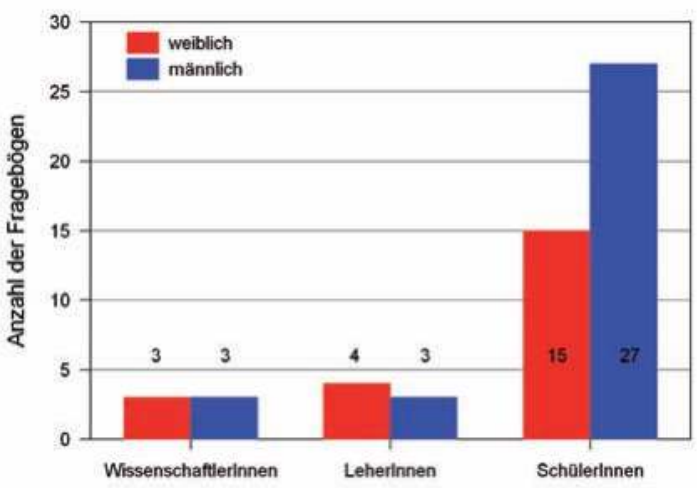

Verteilung der Fragebögen nach Geschlecht 


\section{Schülerbeteiligung}

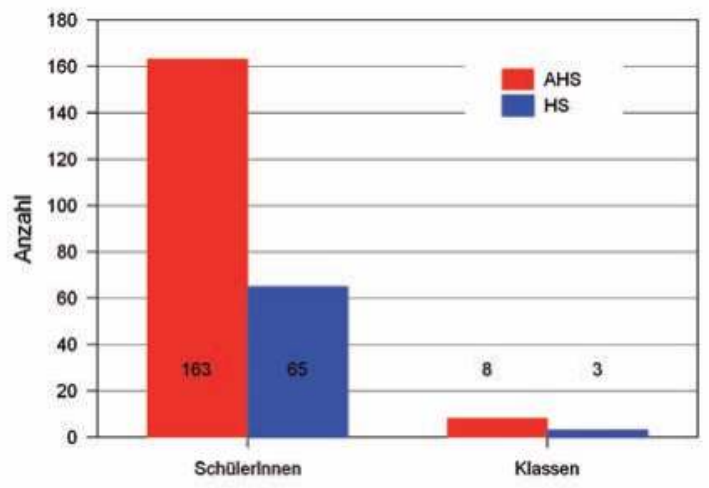

Teilnehmende ScbülerInnen und Klassen unterteilt nach AHS und HS

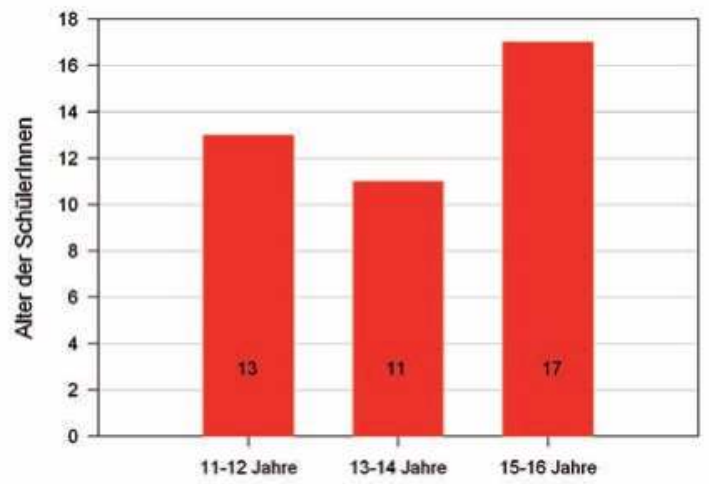

Alter der beteiligten SchülerInnen
Auf die Frage, wie viele SchülerInnen am Projekt teilgenommen haben, antworteten die LehrerInnen, dass insgesamt 163 SchülerInnen aus dem AHS-Bereich und 65 SchülerInnen aus dem Hauptschulbereich (HS Zirl) beteiligt waren. Die Zahl der wirklich an dem Projekt beteiligten SchülerInnen dürfte wahrscheinlich doppelt so hoch sein, also ca. bei 440 SchülerInnen liegen. Die SchülerInnen, die bei der Befragung mitgemacht haben, kamen aus 12 Klassen.

Das Alter der SchülerInnen reichte von 11 bis 16 Jahre. 


\section{Fächerverteilung und Aktivitäten}

Die Fächer der beteiligten LehrerInnen und der WissenschafterInnen hatten einen Schwerpunkt in den Naturwissenschaften (Biologie, Meteorologie, Geographie), aber auch die Politische Bildung und Sprachen (Englisch) waren vertreten.

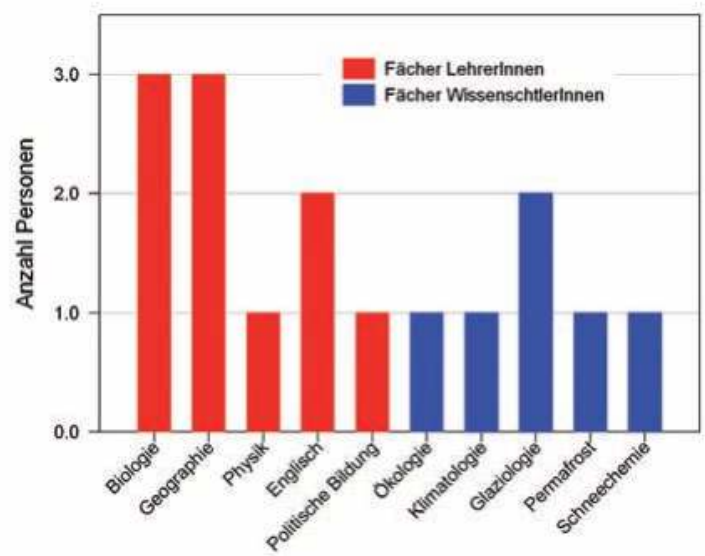

Verteilung der beteiligten Schulfächer

Die Aktivitäten, die während des Projektes gesetzt wurden, waren vielfältig. Exkursionen, Demonstrationen, Experimente, Workshops oder Vorträge wurden genannt. Hervorgehoben wurde die Beteiligung am Kinderkongress (KinKong) im Herbst. Es sind aber alles Aktivitäten, die sich vom normalen Unterricht abhoben.

\section{Erwartungen der LehrerInnen, WissenschafterInnen und Schüle- rInnen}

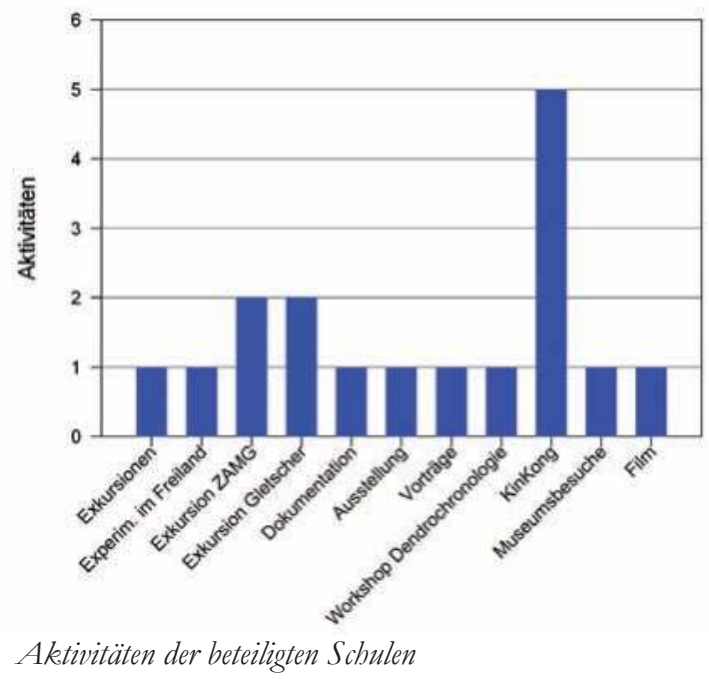

Es gab gemeinsame Erwartungen, aber auch ganz unterschiedliche Interessen am Projekt. Vor allem die SchülerInnen unterschieden sich in ihren Angaben von den restlichen zwei Gruppen.

Wissenszuwachs, etwas Neues erfahren und lernen, neue Erfahrungen gewinnen - diese Kategorien wurden bei allen drei Gruppen genannt: Die LehrerInnen erwarteten sich Horizonterweiterung, mehr Wissen über kalte Regionen (Arktis, Antarktis, Alpen), Klimawandel 
oder Gletscher. Gleiches wurde auch von den SchülerInnen genannt - also auch bei dem Schülerinnen ist ein eindeutiges Interesse vorhanden, etwas zu lernen, aber auch Lernen in Verbindung mit Erfahrung.

Bei WissenschafterInnen stand das Lernen von fachlichen Inhalten nicht im Vordergrund, sehr wohl aber das Lernen im Umgang mit SchülerInnen: Fragen wie „Wie kann man SchülerInnen ansprechen? Auf welchem Level müssen die Inhalt gebracht werden, damit sie für die SchülerInnen verständlich sind?" wurden gestellt, aber auch das Sammeln von pädagogischen und didaktischen Erfahrungen wurde erwähnt.

Bei den WissenschafterInnen steht auch das Bestreben nach Weitergabe ihres Wissens und ihrer Begeisterung für ihr Fachgebiet und für die Wissenschaft im Vordergrund.

LehrerInnen und vor allem auch SchülerInnen wollen auch mehr über die wissenschaftliche Arbeitsweise erfahren. Bei den LehrerInnen fällt auf, dass ihnen auch der Austausch mit anderen LehrerInnen und Schulen wichtig wäre (Finanzen, Know How, Unterrichtsmaterialien), aber auch der fächerübergreifende Unterricht wurde genannt.

Die Erwartungen der SchülerInnen sind teilweise etwas anders gelagert: Wichtig war ihnen durchaus auch etwas Neues zu lernen und zu erfahren, aber Spaß sollte dabei sein. Der Spaßfaktor durfte also nicht fehlen. Aber auch Abenteuer wurde genannt. Manche hatten keine Erwartungen, oder auch sehr ehrlich gesagt: Sie erwarteten sich schöne Tage!!

\section{Haben sich die Erwartungen erfüllt?}

Die Erwartungen haben sich eindeutig bei allen teilnehmenden Gruppen mit fast $100 \%$ erfüllt. Es gab aber auch kritische Anmerkungen: LehrerInnen merkten an, dass es zu wenig Kooperationen zwischen den Schulen und den WissenschafterInnen gab (Zitat: Ich habe ziemlich einsam vor mich hingewurschtelt!) und dass erst am KinKong ein Austausch und ein Kennenlernen möglich war.

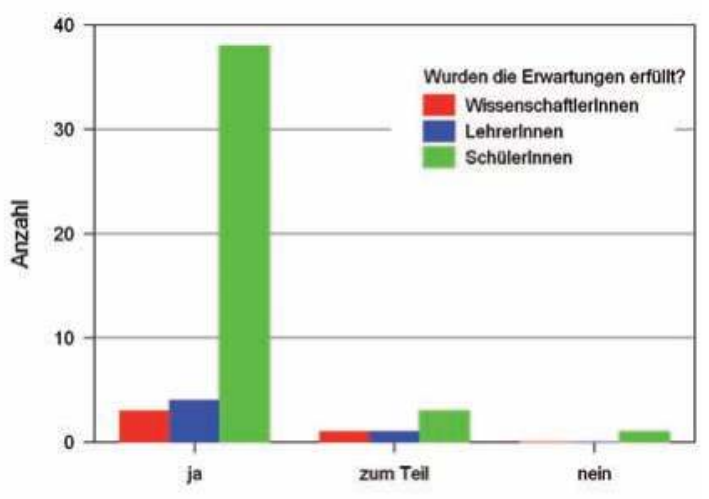

Erfüllung der Erwartungen
WissenschafterInnen bemerkten, dass die SchülerInnen die Inhalte zu wenig hinterfragt hätten, dass sie aber auch von den kreativen Fragen der SchülerInnen überrascht waren und selbst viel gelernt hätten.

Die Rückmeldungen der SchülerInnen waren auch großteils sehr begeistert (Zitate: Ich habe beim KinKong viele SchülerInnen kennengelernt, und in der ZAMG WissenschafterInnen; Interessantes Erlebnis am Gletscher und informativer Kongress an der Uni; Das Projekt hat ermöglicht, Polarforschung hautnah zu erleben; Der Gletscher hat mich beeindruckt; Wir hatten viel Spaß und ich bin auch gescheiter geworden!). 
Sie haben auch erwähnt, dass Forschung durchaus „bodenständig“ sein kann (beispielsweise beim Schneeschaufeln). Die SchülerInnen waren aber auch kritisch und merkten an, dass die Vorträge beim KinKong zu lange waren und dass die Technik am KinKong teilweise nicht funktioniert hat.

\section{Was hat Ihnen (dir) das Projekt gebracht?}

In allen drei Gruppen ist das Wissen gewachsen (Zitate: Mehr Wissen über die Erderwärmung, die Gletscher, den Nationalpark Hohe Tauern, die Veränderung der Natur, Polarregionen, die Arktis und Antarktis oder Flechten).

Aber auch soziale Komponenten wurden erwähnt (Zitate: Ich habe nette, interessante Leute kennen gelernt, die sich mit gleicher Thematik befassen; Nette NiederösterreicherInnen kennen gelernt!; Klassengemeinschaft gestärkt).

Weiters hat sich das wechselseitige Verständnis zwischen den drei Gruppen und ihre jeweiligen Bedürfnisse und Zwänge erhöht (Zitat: Sichtweise und Arbeitsbedingungen von LehrerInnen und SchülerInnen kennen lernen).

Aber auch die emotionale Komponente wurde wieder angeführt, vor allem bei den SchülerInnen spielten Elemente wie Spaß, Freude und Kennenlernen von anderen SchülerInnen eine wichtige Rolle (Zitate: Nette NiederösterreicherInnen kennen gelernt! Irrer Spaß! Lust am Forschen geweckt).

Auf die Frage, was das Projekt den SchülerInnen gebracht hat, erwähnten die LehrerInnen positiv, dass sie einen Einblick in die Welt der Forschung erhalten hätten. Alle Aktivitäten - Exkursionen, Workshops und der KingKong, hier vor allem das Präsentieren vor Publikum, wurden als sehr positiv bewertet.

Kritisch beurteilt wurde von Seiten der LehrerInnen der Zeitdruck, unter dem manchen SchülerInnen gestanden sind, die Finanzierung - für manche Schülerinnen war das Projekt relativ teuer, dass Wissensdefizite bei einigen SchülerInnen bestanden haben, und auch dass die Verständlichkeit der WissenschafterInnen nicht immer gegeben war.

\section{Kontakte zwischen den einzelnen Gruppen}

Die Kontakte zwischen den einzelnen Gruppen wurden als sehr gut bewertet (Zitate: respektvoll, professionell, unkompliziert, angenehm). Die SchülerInnen gaben beispielsweise an, dass WissenschafterInnen auch nur Menschen sind, die Humor besitzen, aber viel arbeiten müssen, ihnen aber der Job auch Spaß macht. Die WissenschafterInnen merkten an, dass sie sehr viel über die Bedürfnisse und Spielräume der LehrerInnen gelernt hätten. In Bezug auf die SchülerInnen gaben sie an, dass sie gemerkt haben, dass SchülerInnen motiviert sind, wenn sie selbst etwas tun können, dass man Fragen einfach und verständlich stellen muss und die SchülerInnen bei ihrem derzeitigen Wissensstand abholen muss. 


\section{Was würden Sie (du) am Projekt verändern?}

Die überwiegende Meinung war, dass man nichts zu verändern brauche. Die Lehrerinnen gaben folgende Veränderungswünsche an: straffere Finanzierung, Finanzierung der Schulexkursionen und auch des Mehraufwandes der LehrerInnen durch das Projekt. Sie wünschten sich mehr Kooperation unter den Schulen und dass der KinKong vor größerem, öffentlichen Publikum stattfinden hätte sollen.

Die WissenschafterInnen hielten fest, dass die Homepage zu spät eingerichtet wurde, die Exkursionen der SchülerInnen aus den Projektgeldern gezahlt werden sollten, dass man SchülerInnen vermehrt an die Uni einladen sollte, was aber aus Kapazitätsgründen oft schwierig ist.

Die Schülerinnen wünschten sich mehr Vorbereitungszeit und bessere Organisation des KinKongs und mehr Öffentlichkeitsarbeit. Auch sehr handfeste Wünsche wurden genannt wie ein Lift auf den Gössenköllesee oder dass das Projekt lieber in der Arktis stattfinden hätte sollen.

\section{Wie war der Zeitaufwand?}

Den Zeitaufwand beurteilten LehrerInnen und WissenschafterInnen unterschiedlich. Während für die Wissenschafterinnen der Zeitaufwand o.k. war, bewerteten die LehrerInnen den Zeitaufwand als sehr hoch, aber dennoch lohnend. Ein Lehrer konkretisierte auch eindrucksvoll, was alles im Rahmen eines Projektes zu tun wäre: Elterninfoabend, Telefonate, Organisation, Feedbackbögen, E-Mailverkehr, Koordination mit Schulterminen, Information von Schulleiter und KollegInnen, Besprechungen mit ProjektlehrerInnen, Gletscherwoche mit 5 Tagen „rund-um-die-Uhr“, Fotos machen, verwalten und versenden, Bericht auf Homepage stellen, Präsentationen für Tag der offenen Tür, Materialeinkauf, Versuchsvorbereitungen und Versuche ausprobieren, Skriptenerstellung,...

\section{Würden Sie wieder teilnehmen?}

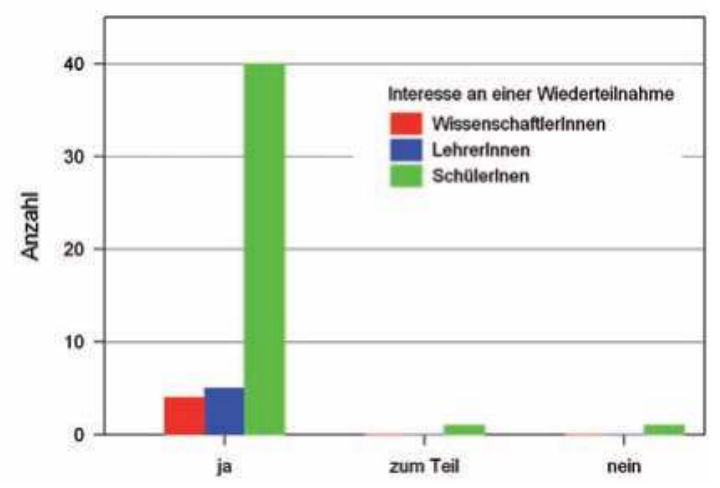

Die Frage wurde eindeutig von allen Gruppen positiv beantwortet.

Interesse an einer Wiederteilnahme 


\section{Zusammenfassung}

Kurz zusammengefasst ergibt sich folgende Quintessenz: Die Zustimmung zum Projekt war bei LehrerInnen, WissenschafterInnen und SchülerInnen sehr groß, alle Gruppen haben sowohl fachlich als auch sozial profitiert, die SchülerInnen auch emotional. Vor allem die Aktivitäten wurden gelobt, und das hohe Engagement aller Gruppen hervorgehoben. Der Zeitaufwand war zwar hoch, aber jede Minute lohnend und das wechselseitige Verständnis zwischen den Gruppen ist gestiegen.

\section{Schlussfolgerungen}

- Genderaspekte: Dass mehr Schüler als Schülerinnen an der Befragung teilgenommen haben, heißt nicht, dass mehr Schüler insgesamt am Projekt beteiligt waren. Man sollte aber immer, und besonders bei Projekten verstärkt darauf achten, dass sowohl Mädchen als auch Buben in ihrer Lebenswelt abgeholt werden.

- Ausbau der Interdisziplinarität: Auch LehrerInnen und WissenschafterInnen aus nicht naturwissenschaftlichen Disziplinen sollten bei Projekten miteinbezogen werden. In diesem Projekt ist es gelungen, mit Fremdsprachen-LehrerInnen zusammenzuarbeiten und den Bereich Politische Bildung und Bildnerische Erziehung miteinzubeziehen.

- Persönlicher Kontakt sehr wichtig: Die einzelnen ProjektteilnehmerInnen müssen sich persönlich kennen lernen, dann funktioniert die Zusammenarbeit. Der KinKong wurde immer wieder lobend erwähnt.

- Verbesserung der Kommunikation: Die Kommunikation könnte durch bessere Nutzung des Internets verbessert werden: Zum schnelleren Info- und Ideenaustausch würde eine Homepage, die gleich am Beginn des Projektes eingerichtet werden sollten, sehr gut geeignet sein.

- Erhöhung des gegenseitigen Verständnisses: Das gegenseitige Verständnis ist durch das Projekt eindeutig gestiegen. So haben beispielsweise WissenschafterInnen erkannt, dass es für LehrerInnen nicht so leicht ist, ein Projekt durchzuführen, da sie ein sehr enges Stundenkorsett haben, und die Stundenstrukturen der Schule sehr einengen und man viel Engagement braucht, um in der Schule ein Projekt durchzuführen.

SchülerInnen erkannten, dass es den WissenschafterInnen Spaß macht zu forschen, dass Wissenschaft aber auch anstrengend ist.

- Anpassung an das Niveau der SchülerInnen: WissenschafterInnen haben erkannt, dass SchülerInnen nicht überfordert werden dürfen. Wichtig ist es, die Fragestellung an das Niveau der SchülerInnen anzupassen und auf Verständlichkeit zu achten, beispielsweise durch Vermeidung von zu vielen Fremdwörtern. Wenn Fremdwörter verwendet werden, sollte die Bedeutung immer erklärt werden. 
- $\quad$ Finanzierung: Bei der Finanzierung wurde angeregt, dass die Kosten für die Exkursionen der SchülerInnen aus dem Projektbudget bezahlt werden sollten. Weiters sollten auch für den Mehraufwand der LehrerInnen Projektmittel vorgesehen werden.

- Verbesserung der Medienarbeit: Die Medienarbeit sollte von Beginn an kontinuierlich betrieben werden, um auch in der Öffentlichkeit die Fortschritte im Projekt aufzuzeigen. Auch hierfür müssten Projektmittel budgetiert werden.

\subsection{Bedingungen der Schulen für FBK-Projekte}

\subsubsection{Bedingungen $B G / B R G$ Lilienfeld Franz Lechner, Michaela Panzenböck; BG/BRG Lilienfeld}

Wir hatten sehr gute Voraussetzungen für die Durchführung des Projekts BiPolar an unserer Schule. Zum einen beteiligten sich drei Lehrer mit unterschiedlichen Fächern daran - Mag. Michaela Panzenböck mit Biologie, Mag. Franz Lechner mit Geographie und Mag. Sigrid Freinberger mit Physik und Chemie, zum anderen arbeiteten wir mit recht engagierten SchülerInnen, die die Wahlpflichtfächer Biologie, Geographie bzw. Physik besuchten. Dies hatte vor allem beim Experimentieren den Vorteil, dass es sich um kleine SchülerInnengruppen handelte. Nur bei den Exkursionen waren größere Klassen mit dabei. Ausflüge an die Uni Wien oder auf den Tirolerkogel, die nur einen Tag dauerten, waren leicht möglich, da es dadurch keine längeren Abwesenheiten von der Schule gab. Zwei mehrere Tage dauernde Gletscher-Exkursionen (Obersulzbachtal und Pasterze) konnten ohne großen bürokratischen Aufwand durchgeführt werden, weil unser Direktor, Mag. Josef Schletz, das Projekt außerordentlich unterstützte. Er fuhr sogar mit zum Kingkong um sich unsere Präsentationen anzuschauen.

Lediglich die technische Ausrichtung der Schule ließ zu wünschen übrig. So scheiterten etwa Messungen an einer Schneedecke am Tirolerkogel an der schlechten Qualität unsere Messgeräte.

\subsubsection{Erfahrungen und Rahmenbedingungen BRG Innsbruck Silke Jung, BRG Innsbruck}

Angesichts der großteils positiven Rückmeldungen der SchülerInnen und den Ergebnissen können wir als LehrerInnen mit diesem Projekt sehr zufrieden sein. Abwechslung vom Schulalltag, ein interessantes Thema, Möglichkeiten für selbstständiges Arbeiten, Erlernen von Präsentationstechniken, enge Kooperation zwischen SchülerInnen, LehrerInnen und WissenschaftlerInnen, fachübergreifende Arbeitsweise, Einsatz von neuen Technologien und wissenschaftlichen Methoden, Teamfähigkeit, all das steht auf der Habenseite. Aneignen von Fachwissen und Förderung von Kompetenzen ergeben ein ideales und modernes Lernumfeld. 
Dennoch bin ich der Meinung, dass an einer AHS (850 SchülerInnen, ca. 90 KollegInnen), wie der unseren, die Rahmenbedingungen für Projektunterricht nicht die besten sind. Ein Projekt dieser Größe ist nur mit Projekttagen einigermaßen zu bewältigen. Kooperationsbereitschaft und Verständnis der Kollegenschaft über entfallene Stunden sind nicht immer gegeben. Auch die Raumsituation ist teilweise unbefriedigend. Schularbeits- und Prüfungstermine, sowie diverse schulische Veranstaltungen erschweren die Koordination. Den Druck der Lehrpläne muss man ohnehin abstreifen, wenn man projektorientiert arbeiten will. Trotz aller Hindernisse werde ich auch weiterhin ein „Projektfreak“ bleiben, weil diese Unterrichtsform den Anforderungen an eine moderne Methodik und Didaktik weitgehend entspricht und meine persönlichen Erfahrungen damit sehr positive sind.

\title{
6.3.2. Erfahrungen und Rahmenbedingungen HS Zirl Markus Freiberger, HS Zirl
}

\author{
Was habt ibr erforscht? - so Mikrozengs \\ Wie siehst du Wissenschaftler - Ja, sind auch nur Menschen
}

Auf die zeitlose Frage auf effektive Wissensvermittlung meinte am 8. 7. 2009 in einem Interview mit der Wissenschaftsredaktion von Bayern II der emeritierte Professor für Entwicklungspsychologie Rolf Örter: „Für unser Bildungssystem hat es (die Selbstkonstruktion des Denkens; Anmerkung der Redaktion) bei Kindern schon große Konsequenzen, denn wenn wir wissen, dass Kinder die Welt konstruieren, dann ist es wichtig, dass sie sich mit der Welt auseinandersetzen, dass sie also Neugier zeigen, diese Neugier behalten, also auch diese wissenschaftliche Neugier. Alle Kinder unter sechs haben eine große Neugier, an dem, was in der Natur vorgeht. Das Problem ist, dass, wenn die Schule zu sehr oder zu viel Wissen vermittelt, die Zeit nicht bleibt, wirklich Forschung zu betreiben und die Erkenntnisse auch in forschender Weise zu erwerben."

Zu dieser Erkenntnis kam schon Mitte des letzten Jahrhunderts Jean Piaget, einer derersten Entwicklungspsychologen. Und nun, fast sechzig Jahren später, fördert unter anderem das Wissenschaftsministerium mit Sparkling Science die Zusammenarbeit von Wissenschaftlern und Schülern, auch das Projekt BiPolar stellte sich das Ziel, dass SchülerInnen forschend die Welt der Polarregionen und das Leben in kalten Lebensräumen entdecken sollten.

Mit einer Klasse mit dem Wahlpflichtfach Ökologie der Hauptschule Zirl hatten wir die Möglichkeit dies über einen Zeitraum von über zwei Jahren zu erproben. Zweimal waren die Kinder auf der Limnologischen Station am Gossenköllesee, zweimal im Universitätszentrum in Obergurgl, von wo aus sie Exkursionen zum Gletscher machten. Außerdem besuchten sie einen Zoologen auf der Uni Innsbruck und konnten im Freigelände die Aufzuchtbecken, der von ihnen erforschten Kaiser-Max-Forelle kennen lernen. Dr. Medgyesi brachte es, als wir ihm erzählten, was wir mit den SchülerInnen machten, auf den Punkt: „So hätte ich auch gerne Schule erlebt.“ Fast wortgleich war die Aussage eines ORF Mitarbeiters, der uns bei der Schülerexpedition begleitete. Jeder Außenstehende, nicht zuletzt die Juroren des IMST Awards, der für das Projekt als letzte Auszeichnung nach dem Sparkling Science 
Preis verliehen wurde, war von dem, was wir speziell mit so jungen Schülern unternahmen, begeistert. Wobei der Funke der Begeisterung von einem Zehnjährigen, der aufgeregt zum ersten Mal Proben am Gletscher nimmt, unvermittelt auf einen überspringt, ob Lehrer, Wissenschaftler oder zufällig vorbei kommender Wanderer. Meiner Beobachtung nach gibt es keine Zeit, in der junge Menschen so interessiert sind am Forschen, wie im Alter zwischen zehn und zwölf. Das weiß natürlich schon lange die Wirtschaft. In zwölf von mir befragten Klassen gaben die Erst- und Zweitklässler an, dass sie sich zu Weihnachten oder einem ähnlichen Anlass ein Mikroskop (der Inbegriff des Forschungsinstrumentes für Kinder) gewünscht hätten. Freilich wollten sie eigentlich eine Stereolupe, denn unter dem Durchlichtmikroskop sahen die Jungforscher nur einen schwarzen Fleck, wenn sie - oft sogar ohne Objektträger - z.B. eine ganze Fliege auf den Objekttisch gelegt hatten. So werden von fast allen Kindern, die Fertigpräparate ein, zwei Mal angeschaut und dann verschwindet das Mikroskop für immer in einem Schrank im Kinderzimmer. Aber es gibt sie, jene Kinder, die wirklich forschen, sich die Mühe nehmen auch ein Buch zu lesen, um zu lernen, wie man ein Präparat anfertigt, aber die Anzahl ist sehr gering. Pro Klasse mit 8-10 Mikroskop-Forschern meist nur einer oder eine.

Bleiben wir bei der These, die heute fast unbestritten von Schule und Entwicklungspsychologie vertreten wird, nämlich, dass es weitaus besser ist Kenntnisse forschend zu erwerben als nur, im schlimmsten Fall im Frontalunterricht, unkritisch zu reproduzieren. Hierzu wäre eine Evaluierung notwendig. Ich erlaubte es mir mit den Mitteln der Schule zu überprüfen, sprich mit so genannten Lernzielkontrollen. Das Ergebnis war verblüffend und ernüchternd zugleich. Die SchülerInnen hatten nach einem Jahr genau so viel von dem vergessen, was sie erforscht hatten, wie von dem, was sie im übrigen Unterricht gelernt hatten. Also alles umsonst? Nicht ganz. Die Fertigkeiten, die sie bei den Exkursionen erlernten, beherrschten sie noch und die Sicherheit, mit der sie (nunmehr als Drittklässler) Bärtierchen unter der Stereolupe aus einer Probe in einer Petrischale mit einer Pipette zwischen dem Gesteinsstaub herausfischten, um sie in einem mikroskopischen Präparat genauer zu betrachten, waren nicht nur geblieben, sondern hatten sich sogar verbessert. Auch die Begeisterung und Neugier war geblieben, als Lehrer musste man nur mehr einmal mehr staunend - beobachten, was auf den Blättern neben den Instrumenten von Zwölfjährigen gezeichnet wurde.

Meine Lieblingsdefinition von Intelligenz ist Folgende: Intelligenz ist die Fähigkeit, Probleme zu lösen. Und diese Fähigkeit wurde mit Sicherheit durch das Projekt BiPolar bei unseren SchülerInnen gefördert. Außerdem blieb die Freude und die Begeisterung am forschenden Lernen, nach Abschluss von BiPolar wollen ausnahmslos alle SchülerInnen, dass es irgendwie weitergeht, am liebsten mit dem geplanten Projekt TriPolar, das sich unter anderem mit der zukünftigen Erforschung und der Suche nach Leben in den Eispolen des Mars beschäftigt. 


\subsection{Erfahrungen der SchülerInnen}

„Im Großen und Ganzen kann ich sagen, dass das Sparkling Science Projekt „BiPolar" ein voller Erfolg war. Ich finde solche Projekte für Schulklassen sollte es öfter geben, da dies in meinen Augen sebr motivierend für uns Jugendliche ist."

Lisa Gamper

„Alles in allem war dieses BiPolar Projekt ein voller Erfolg! Der gesamte Klasse machte es sehr viel Spaß über dieses Thema zu recherchieren und gemeinsam Informationen zu sammeln. Meiner Meinung nach sollte man öfter solche Projekte durchführen, da sie sehr motivierend und lehrreich sind und auch die Klassengemeinschaft zusammenschweißen!"

Philipp Zluga

„Vorerst möchte ich das ganze Projekt loben, da es lehrreich war und gut umgesetzt wurde. Begonnen hat alles mit einem Vortrag der Biologin Birgit Sattler. Darauf folgten dann einige Projekttage, die wir dazu nütəten uns zu informieren und eine Powerpoint-Präsentation zu erstellen. Dazu kam auch ein Projekttag, an dem wir ins Kühtai fubren und beispielsweise den zugefrorenen Gossenköllesee anbohrten. Als Ergebnis hielten wir einen Vortrag am KinKong in Wien. Insgesamt war es ein tolles Projek.t. Es gibt kaum einen Punk.t, den man kritisieren könnte, außer dass die Schlusspräsentation wegen technischer Schwierigkeiten nicht wie vorbereitet verwendet werden konnte. Ich würde es sehr unterstützen, wenn wir weitere Projekte in Angriff nehmen würden. "

Lukas Grubwieser

„Mehrere Projekttage für Recherchearbeit, eine Exkursion ins Kühtai, in den letzten Tagen fieberhafte Vorbereitungen für die Präsentation und anschließend die Klassenreise zum KinKong in Wien machten das „Projekt BiPolar" zu einem umfangreichen, aber nichtsdestotrotz tollen Projekt! Auch die Betreunng während des Projektes war optimal, die Forscherin Birgit Sattler versteht es, Jugendliche für die Polargebiete zu begeistern.

Alles in allem hat das „Projekt BiPolar" die gesamte Klasse überzengt. Wir hoffen, nächstes Jahr ein ähnlich tolles Projekt machen zu können!"

Thomas Hirn

„Kurz darauf machten wir einen Ausflug ins Kühtai zum Gossenköllesee. Dort befindet sich die kleinste limnologische Forschungsstation der Welt. Aus dem See nabmen wir Eis- und Wasserproben, die wir nachber im Labor untersuchten.

Für mich war das ein wichtiger Punkt, weil wir dort viel über Kleinstlebewesen im Eis und Wasser gelernt haben."

Veronika Kinzner

„Um einen praktischen Eindruck zu bekommen, konnten wir im Rabmen einer Exkursion zur limnologischen Forschungsstation am Gossenköllesee unter anderem Wasserproben nehmen und bekamen so einen Einblick in die faszinierende Welt der Gletscherforschung. "

Fabian Laimer 


\section{Coole Forschung}

„Nach zablreichen Stunden theoretischen Arbeitens wollten wir unser Wissen in die Praxis umsetzen. Mithilfe der Forscherin Birgit Sattler, die schon öfter in der Antarktis gewesen war, machten wir uns auf den Weg ins Kühtai, zum Gossenköllesee. Schon der Weg dorthin bereitete uns großen Spaß, da wir durch meterhohen Schnee stapfen mussten, um schlussendlich an der Forschungsstation anzukommen. Der kleine Bergsee war von einer dicken Eisschicht bedeck.t. Es dauerte einige Zeit, bis wir es schafften, ein Loch in die Eisschicht zu schlagen. Nun war es möglich, dem See Wasserproben zu entnehmen, die wir anschließend unter dem Mikeroskop untersuchten."

Johanna Lercher \& Nina Payr

„An diesem Projekt teilzunehmen war, meiner Meinung nach, eine gute Entscheidung von unserer Lehrerin. Es war interessant, so viel über die Pole und den Lebensraum, den sie bieten, zu erfahren. Die Projekttage waren auch nicht nur wegen der ausgefallenen Unterrichtsstunden, sondern auch wegen dem interessanten Thema gut."

Thomas Mader

„Dieses tolle Projekt startet mit einem Bericht, über die polaren Zonen der Welt, von Birgit Sattler. Sie konnte uns sofort mit diesem Vortrag begeistern. Zur Krönung der Projekte durften wir mit auf die Hochalpine Forschungsstation für Ökologie am Gossenköllesee im Kühtai Tirol."

Fabian Mair

„Geographie sowie Geschichte und Politik. Nachdem unsere Gehirne bereits gestopft voll mit BiPolarität waren, folgte ein Ausflug ins Kübtai (Gossenköllersee), wo weder Eiseskälte noch Handschubverwechslungen dem Schülerschen Lerndrang (oder vielleicht doch ein Drang nach heißem Tee und mitgebrachten Frankfurtern) die Kraft nehmen konnte. Mit Mikroskop und Eisbohrer wurden Wasserproben entnommen, wobei die nicht beschäftigten 14 Schüler (es können rein räumlich eben nicht 19 Personen zugleich ein Loch bohren) sich anderweitig im Schnee beschäftigten. Obne Zweifel das Highlight des Projektes."

Alexander Sarg

„Für junge Menschen sind solche Projekte eine gute Möglichkeit, sich weiter zu bilden. Darum ist es auch gut, wenn diese unterstützt werden. Das Projekt BiPolar war eine tolle Erfahrung, ich würde jederzeit wieder daran teilnehmen. "

Madlen Schranz

„Das Projekt war zwar mit viel Arbeit verbunden, aber auch mit sehr viel Spaß. Alles in allem war es wirklich interessant und ich hoffe, dass wir die Möglichkeit haben, an weiteren Projekten dieser Art teilnehmen zu dürfen."

Eva Schwärzler

„Hallo, ich bin Jonas Vasak, bin 14 Jahre alt und gehe in die 8. Schulstufe der Erlebnisschule Mödling. Ich möchte Ihnen in diesem Text meine Eindrücke zum Projekt BiPolar näherbringen. 
Ich habe in einer Gruppe gearbeitet, die sich damit befasste, wie sich die Gletscherschmelze auf das Klima auswirk. und umgekehrt das Klima auf den Gletscher. In dieser Gruppe arbeiteten außerdem noch Julian Buchinger, Julian Rössl und Aniko Braumüller. Die Arbeit in dieser Gruppe hat mir sebr gut gefallen. Wir haben zusammen eine Power Point Präsentation erstellt, die wir dann am KinKong, dem Kinderkongress im Biozentrum der Universität Wien und bei der Eröffnung unserer Ausstellung in der Schule „Der Gletscher lebt... wie lange noch?" vorgestellt haben.

Die Gletscherwoche im September 2008 am Goldbergkees am Hohen Sonnblick fand ich sehr interessant. In dieser Woche haben wir - zusammen mit dem berühmten Wissenschaftler Wolfgang Schöner und seinem Team - folgende Messungen durchgeführt: mit GPS sind wir den Gletscherrand abgegangen, um herauszufinden, wie weit sich der Gletscher zurückgezogen hat. Weiteres haben wir eine fünf Meter tiefe Dampfbohrung gemacht, um herauszufinden, wie tief der Gletscher ist und ein Schneeprofil gegraben, um herauszufinden, wie der Schnee beschaffen ist.

Alles in allem hat mich BiPolar sehr interessiert und angesprochen. Fazit: gutes Projekt!"

Jonas Vasak

„Ich heiße Jakob Schöner, bin 14 Jahre alt und bin Schüler der Erlebnisschule und Teilnehmer beim BiPolar. Ein Jahr nach dem Beginn des BiPolar fuhren wir mit der ganzen Klasse eine Woche auf den Gletscher. Dort machten wir viele interessante Versuche. Außerdem lernten wir den Dampfbohrer kennen, mit dem man durch Wasserdampf Löcher bohrt. Danach steckt man Pegel (Plastikstangen) in das Eis. Weil die Gletscher immer mehr schrumpfen, kann man anhand der Pegel einen genauen Gletscherrück.zug sehen. Eine andere Methode um den Gletscherschwund zu messen, ist mit einem GPS über mehrere Jahre hinweg das Ende einzumessen.

Auch wir konnten den Versuch machen. Wir hatten viel Spaß am Gletscher. Als wir wieder zurück waren, mussten wir unsere Ergebnisse auswerten. Ca. 3 Monate später fand der KinKong an der Uni Wien statt. Beim KingKong sahen sich alle Schulen und präsentierten ihre Ergebnisse.

Ich fand das BiPolar sehr interessant und hoffe, dass es wieder so ein Projekt in der Art gibt."

Jakob Schöner

\subsection{Kooperation in BiPolar - Ein Blick von außen auf den Brückenschlag zwischen Forschung und Schule Franz Radits, Anna Streissler, Franz Rauch Austrian Educational Competence Centre}

\subsubsection{Ziele der Evaluation}

Dieser Abschnitt geht der Frage nach: Was wollte das Evaluationsteam im Auftrag der Projektleitung über das Projekt wissen und wie haben sich diese Fragen im Verlauf des Projekts verändert und konkretisiert.

Vor Beginn des Projekts entwickelten Projektleitung und externes Evaluationsteam in zwei Workshops Forschungsfragen, die sich an den Projektzielen orientierten. 
Diese Fragen betrafen 11 Themenbereiche:

1. In wie fern können SchülerInnen die Fragen von WissenschafterInnen beeinflussen? Wo ist Partizipation von SchülerInnen im Forschungsprojekt möglich?

2. In wie fern erleben die SchülerInnen die Mitarbeit in praktischen Forschungs-Settings als motivierend?

3. Wie sind die Rahmenbedingungen in der Schule für Forschungs-Bildungs-Kooperationen (FBK) im Projekt BiPolar?

4. Wie gelingt fächerübergreifender Unterricht im Rahmen von BiPolar?

5. Welche Bilder haben ForscherInnen von Fachunterricht und Schule? Welche Bilder haben SchülerInnen und LehrerInnen von ForscherInnen und Forschung? Wie ändern sich die Bilder im Projektverlauf?

6. Was lernen die SchülerInnen (und LehrerInnen) bei BiPolar über die Kultur des wissenschaftlichen Forschens (sozialwissenschaftlicher Aspekt von Forschung)? Was lernen die SchülerInnen (und LehrerInnen) bei BiPolar über aktuelle Polarforschung (naturwissenschaftlich-inhaltlicher Aspekt von Forschung)?

7. In wie fern erhöht FBK in BiPolar die gesellschaftliche Wirkung von Klimaforschung? In wie fern werden SchülerInnen als MultiplikatorInnen wirksam?

8. In wie fern gelingt der Austausch zwischen LehrerInnen untereinander und zwischen LehrerInnen und PolarforscherInnen?

9. Welche Erfahrungen machen LehrerInnen bei der Mitarbeit an der Forschung in BiPolar?

10. Welche Erfahrungen machen SchülerInnen bei der Mitarbeit an der Forschung in BiPolar?

11. Welche Wirkungen hat die Mitarbeit an BiPolar auf den künftigen Unterricht der LehrerInnen?

Nicht alle dieser Fragen wurden gleichwertig gewichtet. Der Prozessverlauf und die Schwerpunktsetzung der Projektleitung bestimmt die Wertigkeit in dieser Studie. Diese Focussierung ist aus den Aufgabenstellungen für die Schlussevaluation (Herbst 2009) erkennbar:

1. Erfolge und Misserfolge von BiPolar

2. Wie hat die Zusammenarbeit von LehrerInnen, SchülerInnen und WissenschafterInnen im zeitlichen Verlauf von BiPolar funktioniert? Welche Rollen nahmen welche Personen wann ein? Wer konnte wie von wem lernen? Was war für das Lernen voneinander förderlich, was hinderlich?

3. Sollte ein ähnliches Projekt wieder durchgeführt werden, was sollte stärker beachtet bzw. gegenüber BiPolar verändert werden?

4. Wie hoch ist die Motivation der Beteiligten, sich wieder an einem ähnlichen Projekt zu beteiligen?

5. Woran wurde konkret geforscht und wie? 


\subsubsection{Forschungsdesign}

In diesem Abschnitt gehen wir der Frage nach, welche Überlegungen es zu den Forschungsmethoden im Verlauf der Begleitforschung gab und wie diese auf Grund praktischer Umstände angepasst wurden.

Im ersten Planungstreffen zwischen Evaluationsteam und Projektleitung wurde ein qualitatives Forschungsdesign, das formative (projektbegleitend) und summative Elemente (nach Projektabschluss) enthält, vereinbart. Geplant war, dass bei einem Halbzeitmeeting die Beobachtungen der EvaluatorInnen und die Erfahrungen des Leitungsteams reflektiert werden.

Die ursprünglich entwickelten 11 Fragen erforderten drei unterschiedliche Ansätze bei der Datenerhebung:

- „Prä-post“ Datenerfassung, wenn Veränderungen der Akteure während des FBKProzesses zu untersuchen sind.

- Summative Datenerhebung zur Situation am Ende des Projekts

- Prozessbegleitende (formative) Datenerhebung

Nach Rücksprache mit der Projektleitung wurde die Zwischenerhebung, ein formatives Element der Evaluation, durch eine projektinterne Erhebung ersetzt. (s. Wolfgang Schöner: Bildungsinitiative Polarforschung - Lernen und Forschen mit FERMAP - BiPolar. Endbericht. GZ 37.539/1-II/4/2007, Kapitel 6.2; 19.11.2009) Weiters wurde vereinbart, dass verstärkt die Perspektive der LehrerInnen erfasst erfasst werden sollte. Dazu wurden zwei Möglichkeiten erörtert:

a) LehrerInnen als ForscherInnen: Sie entwickeln selbst Fragen bezogen auf ihre konkrete Situation, erheben selbst Daten im Unterricht, werten diese selbst aus und verfassen selbst kurze Berichte. Dies erfordert Begleitung und Austausch unter den LehrerInnen (Communities).

b) LehrerInnen sammeln Daten und stellen diese dem Evaluationsteam zur weiteren Auswertung zur Verfügung.

Die Ergebnisse der von Anna Streissler durchgeführten teilnehmenden Beobachtung am Modul 1 wurde als Steuerungswissen an die Projektleitung weitergeleitet und ist die Basis für die Eingangsevaluation. Das Einbeziehen der LehrerInnen in die formative Evaluation (nach dem Konzept der Aktionsforschung) wurde in Modul 1 vorgeschlagen, im weiteren Projektverlauf jedoch nicht mehr aufgegriffen.

Nach dem Modul 1 erfolgte die weitere Kommunikation über E-Mail. Im Herbst 2009 wurde die Ausgangsevaluation zwischen Projektleitung und Evaluationsteam konkretisiert. Die ursprünglich erwogenen formativen Evaluationselemente entfielen dadurch. 


\subsubsection{Abschlussevaluation}

\section{Focus und Methoden}

Ziel der abschließenden Evaluation war es, die Erfahrungen der drei beteiligten Akteursgruppen im Projekt BIPLOAR zu erfassen. Dazu wurden sechs Kategorien entwickelt: 1) gelungene Aspekte, 2) weniger gelungene Aspekte, 3) die Zusammenarbeit der Akteure, 4) Vorschläge für Veränderungen, 5) die Bereitschaft wieder teilzunehmen und 6) weitere für die Kooperation interessante Rückmeldungen.

Diese Kategorien erfassen nur teilweise die ursprünglichen Evaluationsziele. Die Ursache für diese Einschränkung liegt in der stark beschränkten Möglichkeit nach Abschluss des Projekts von allen Akteuren ausreichend Daten zu erheben. Die Ursachen dafür liegen wahrscheinlich auf zwei Ebenen:

- Das Projekt endete für die unterschiedlichen Akteursgruppen zu unterschiedlichen Zeiten. Daher kam der Zeitpunkt für die Abschlussevaluation für einen Teil der SchülerInnen zu spät. Auch ein Teil der WissenschafterInnen war in dieser Zeit bereits im Rahmen anderer Projekte in entlegenen Erdteilen mit wenig Kommunikationsinfrastruktur unterwegs.

- Es herrschte Unklarheit bei WissenschafterInnen, LehrerInnen und SchülerInnen, warum Evaluationsdaten notwendig sind und der Mangel verbindlicher Übereinkünfte, was damit geschieht.

Für die Abschlussevaluation sollten alle LehrerInnen und alle WissenschaftlerInnen befragt werden. Je nach der Anzahl der in BiPolar vertretenen Schultypen und Altersgruppen sollte eine repräsentative Anzahl SchülerInnen befragt werden.

Es wurde ein qualitativer Fragebogen konzipiert, der die fünf wichtigsten Fragen des Projektteams (siehe oben) aufgriff und der auf die jeweilige Zielgruppe (WissenschafterInnen, LehrerInnen und SchülerInnen) angepasst wurde. Der Fragebogen wurde nach dem letzten Modul Mitte Oktober 2009 an alle beteiligten WissenschafterInnen und LehrerInnen ausgeschickt. Einige LehrerInnen übernahmen die Aufgabe, die Bögen an ihre SchülerInnen weiterzuschicken.

Der Rücklauf der Fragebögen war insgesamt niedrig und entspricht ungefähr den Zahlen der Zwischenevaluation. (3 WissenschafterInnen, 6 LehrerInnen aus 6 Schulen, 36 SchülerInnen aus 4 Schulen). Das Material erscheint jedoch ausreichend, um über die von den Akteuren und Akteurinnen berichteten Erfahrungen Rückschlüsse auf die Qualität der Kooperation machen zu können.

In der nachfolgenden Darstellung der Ergebnissen sind die Aussagen von WissenschafterInnen und LehrerInnen großteils im Wortlaut übernommen und dort, wo es klare inhaltliche Übereinstimmungen gibt, zusammengefasst. Die Aussagen der 36 SchülerInnen sind teilweise inhaltlich gruppiert und zusammengefasst.

Alle Aussagen wurden anonymisiert. Um Verweise auf die Originaldaten nachvollziehbar zu machen, wurden die 3 Fragebögen der WissenschafterInnen als W1, W2 und W3 
bezeichnet, die sechs Fragebögen der LehrerInnen analog L1-L6 und die Fragebögen der SchülerInnen S1-35. Die Originalzitate sind der leichteren Lesbarkeit kursiv gesetzt. Um die Anonymität einzelner SchülerInnen zu bewahren, wurden an einigen wenigen Stellen Hinweise auf konkrete Personen (v.a. LehrerInnen) durch Umschreibungen ersetzt.

\section{Ergebnisse der Schlussevaluation}

\section{Gelungene Aspekte von BiPolar}

Die Versuche bzw. Experimente strichen 15 SchülerInnen als besonders positiv hervor. Dabei erwähnten sie einzelne Fächer und einzelne Versuche, nämlich den Verrottungsversuch (2 Nennungen), die Experimente zu Eisbärfell und Eisbärohren (4 Nennungen). Die Vorträge der WissenschafterInnen fanden hohen Anklang, denn diesen Aspekt erwähnten 13 SchülerInnen.

Die Ausflüge und Exkursionen fanden sechs SchülerInnen besonders interessant. Fünf SchülerInnen lobten die Zusammenarbeit und den Austausch zwischen den Akteuren, hier zwei Aussagen: „Die Zusammenarbeit zwischen den Forschern und Schülern, vor allem die Vermittlung von Know-how." (S1) Der Kinderkongress, ein „Zusammentreffen aller mitwirkenden Schüler", war für vier SchülerInnen ein Highlight. Vier SchülerInnen erinnerten sich an die besonders gut verlaufenen Interviews mit den Tierpflegern im Tiergarten Schönbrunn.

Drei SchülerInnen erwähnten Projekttage besonders positiv. Hier zwei Aussagen: „Ich bin der Meinung der Projekttag im Kübtai war besonders gelungen, weil wir ansonsten zum Großteil in einem geschlossenen Raum gearbeitet haben. Und das war etwas anderes aber genau so informativ.“ (S2) „Der Projekttag mit Bio, Chemie und Physik, an dem wir Versuche zur Anpassung der Tiere in Polarregionen gemacht haben." (S25)

Zwei SchülerInnen lobten das fächerübergreifende Arbeiten. Ebenfalls zwei SchülerInnen erwähnten den Besuch im Tiergarten Schönbrunn am Arktistag als besonders positiv, „weil wir den Leuten die Arktis näher bringen konnten“ (S28). Zwei SchülerInnen meinten auch, sie hätten sich ,intensiv mit dem Thema beschäftigt" (S31, S32), zwei andere, dass es ,gan z“" oder ,sehr" „interessant" war (S27, S29).

Weitere Einzelantworten waren:

- Eindrücke aus anderen Gebieten zu sammeln.“ (S3)

- „Finanzielle Unterstützung vom Staat“ (S6)

- Dass sich alle Kinder für eine Sache eingesetzt haben.“ (S13)

- „Bilder im Festsaal“ (S19)

- Das Ergebnis unseres Projektes“ (S24)

- „Aus meiner Sicht ist das Projekt BiPolar gut gelungen, weil die Stationen gut und interessant aufgebaut waren. Auch gut war, dass wir uns die Informationen selber erarbeitet haben." (S34)

Ein/e SchülerIn berichtete über die Erhöhung des inhaltlichen Verständnisses: „Meine Verständlichkeit für die Polarregionen ist gestiegen und ich habe deswegen meinen Lebensstil leicht geändert." (S9) 


\section{Coole Forschung}

Alle LehrerInnen erachteten die gute Zusammenarbeit zwischen WissenschaftlerInnen und Schulen als besonders gelungen. Dies wurde teilweise konkretisiert: „Berufsfeld kennenlernen; Fragen und Inhalte - direkt im Austausch mit Wissenschaftlern"(L3).

Für drei Lehrpersonen war die Teilnahme an einem wissenschaftlichen Kongress besonders attraktiv. Eine Person nannte es ,großer Ansporn und ,Zuckerl' zugleich" (L4).

Weitere positive Aspekte wurden genannt, wobei fachdidaktische und methodische Aspekte große Bedeutung einnahmen:

- „Eintauchen in die Materie“(L3)

- $\quad$ „Nengierde vermitteln“(L3)

- „SchülerInnen für neue Themen zu gewinnen“(L5)

- „Diese zu neuen Arbeitsmethoden zu fübren“(L5)

- „Das Ziel, die Förderung der Zusammenarbeit Schüler-Lehrer-Wissenschaftler (auch gedacht als -innen) wurde erreicht."(L6)

- Das zweite Ziel, forschendes Lernen, konnten wir mit unserem Projekt perfek.t umsetzen. “(L6)

Aus der Sicht der WissenschafterInnen ist Folgendes gut gelungen:

- „Der Brückenschlag zwischen Wissenschaft und Schule“(W1)

- „Der Kontakt zwischen Lehrern/Wissenschaftern/Schülern“ (W1)

- $\quad$ „Der Austausch zwischen Schülern und Wissenschaftern in Bequg auf Erstellung von Forschungsaufträgen bzw. eigenen Fragen" "(W1)

- „Die (erwünschte) Interdisziplinarität (Biologie, Meteorologie, Physik, Geographie, Englisch, Bildnerische Erziehung, etc.)"(W1)

- „Kinder für Forschung zu begeistern“ (W1)

- „Schülern ein (realistisches) Bild einer Universität zu geben“ (W1)

- Nachbaltigkeit im Unterricht zu schaffen“(W1)

- Durch Preise, Pressemeldungen und Anerkennung konnten die Schüler vermehrt motiviert werden.“ (W1)

- Exkursionen bzw. OutdoorAktivitäten gemeinsam Schüler-Lehrer-Wissenschafter. “(W2)

- $\quad$ „Gemeinsames Arbeiten am Gletscher (飞.B.) vermittelte viel von der Arbeit eines Wissenschaftlers, Schüler konnten selber Hand anlegen, waren Mitarbeiter. Abwechslung zum Schulalltag und auch zu anderen ,normalen" Exkursionen. "(W2)

- Motivation der Projektpartner“(W3)

- „Interesse aller Projektteilnehmer für den Klimawandel und Vertiefung des Wissens“(W3)

- „Kinderkongress“(W3)

- Gleiche Augenhöhe der Projekteilnehmer“ (W3)

- „Interesse für Wissenschaft wecken“(W3) 


\section{Weniger gelungene Aspekte von BiPolar}

Sechs SchülerInnen antworteten, dass „alles gut" oder „gelungen“" war (S2, S10, S21, S24), „nichts" misslungen war (S26) oder es "keine Komplikationen" gab (S6). Ein/e SchülerIn meinte, „Es ist bei uns nur ein Versuch nicht gelungen“(S8).

Die anderen Antworten auf diese Fragen bezogen sich auf vielfältige Einzelaspekte. Drei SchülerInnen bezogen sich auf den Kinderkongress. Dieser war ,ziemlich anstrengend und zach" (S3), ,zu langwierig und [...] mit der Zeit ein bisschen langweilig“"(S4). „Es hat zuerst geheißen, dass beim KinKong auch der ORF anwesend ist, war dann aber nicht so." (S5)

Zwei SchülerInnen bezogen sich auf „Vorträge" (S13, S14), in einem Fall explizit beim Kinderkongress: „Die Vorträge sind nicht gut gelungen, da alle Scbüler beim KinKong dasselbe referiert baben “(S13). Zwei weitere SchülerInnen (S17, S18) kritisierten die mangelnde „Zusammenarbeit zwischen den Fächern" (S17).

Uneinigkeit herrschte unter den SchülerInnen, ob das Projekt zeitlich zu kurz (S35) oder zu lange (S7, S36) dauerte. Weitere Antworten waren:

- Die Vermittlung mit Menschen außerbalb vom Projekt"(S9)

- "Das theoretische wie ₹.B. die ganzen Arbeitsblätter. Zu viele Fragen“(S12)

- „Dass die Ausflige ₹.B. in die Uni oder in den Tiergarten meistens am Anfang oder am Ende ziemlich chaotisch waren" $(\mathrm{S} 31)$

- „dass manche es gan₹ lustig fanden und einfach so rumgesessen sind und nicht viel mitgemacht haben “ (S29)

- „dass der Ausflug in den Tiergarten etwas komisch war" (S30)

- „Die direkte Wissensübertragung hat nicht immer gut geklappt, speziell zwischen Lehrern und Scbülern" (S16)

12 SchülerInnen beantworteten diese Frage nicht.

Die Meinungen der LehrerInnen über weniger gelungene Aspekte fielen unterschiedlich aus. Eine Lehrperson identifizierte keine negativen Aspekte im Projekt, eine zweite sah „wenig Änderungsmöglichkeit, da ich als Nicht-Naturwissenschaftler in diesem Projekt keinen so großen Gestaltungsspielraum hatte."(L4)

Zwei LehrerInnen kritisierten die organisatorischen Schwierigkeiten bei der Durchführung von Projektunterricht, „das Schulkorsett ist viel enger, als Außenstehende dies vermuten“(L5). LehrerIn L6 wies darauf hin, dass dies ein generelles Problem wäre und nicht spezifisch für BiPolar. Zwei weitere Lehrpersonen kritisierten die mangelnde Zusammenarbeit unter den Schulen (L1, L5).

Andere Aspekte waren:

- $\quad$ „Als Lehrer habe ich die Machbarkeit bestimmter Ziele falsch eingeschätz̨t - das heißt: als Lehrer wollte ich teilweise zu viel!" (L5)

- „Auftakt-Workshop für LebrerInnen (Inhaltt)" (L2) 


\section{Coole Forschung}

- $\quad$ „KINKONG könnte vor größerem Publikum stattfinden, das sich nicht mit dem Thema befasst hat. Wissenschaftler könnten als Vortragende in den Kongress stärker eingebunden werden" (L4)

- Die Präsentation der Arbeiten ist nur teilweise gut gelungen” (L5)

- „Die Einbettung der vielen Teilaspekte in ein gesamtes Ergebnis ist mir zu wenig gut gelungen.” (L5)

Aus der Sicht der WissenschafterInnen ist Folgendes weniger gut gelungen:

- Die geographische Aufteilung im Projekt war ebenso ,bipolar;, es kristallisierten sich 2 Kernzonen heraus (BiPolar Ost und BiPOlar West), was jedoch unumgänglich ist. Es ist unmöglich, als Beteiligter in Tirol eine Schule in Ostösterreich (oder umgekehrt) zur Zufriedenheit zu betreuen, da eine gewisse Flexibilität und auch Nähe zu den Schülern wichtig sind. Diese 2 Zonen waren jedoch kein Nachteil, aber der uneingeschränkte Austausch in ganz Österreich kann so nicht funktionieren." (W1)

- Die SchülerInnen sollten selber Forschungsfragen entwickeln und bearbeiten. Dies gelang nur teilweise. (W2, W3)

\section{Zusammenarbeit der Akteure in BiPolar}

Einige SchülerInnen machten recht allgemein formulierte Aussagen, z.B.: „Die Ausflüge waren gut und super" (S35).

In vielen allgemein gehaltenen Aussagen wurden die LehrerInnen und WissenschafterInnen als sehr unterstützend erlebt, z.B.:

- „Lehrer und Schüler konnten von Wissenschaftlern viel lernen. Man kann einige Sachen besser nachvollziehen."(S3)

- Unsere Lehrer unterstützen uns bei allen Projekten und auch die Wissenschaftler zeigten uns wie die Geräte funktionierten. Es war sehr viel Interessantes dabei und ich konnte einiges lernen. "(S5)

- „Die Lehrer unterstütəten uns, wo sie nur konnten. Natürlich, die Basis für etwaige Präsentationen mussten schon wir Schüler ausarbeiten." (S7)

- "Zusammenarbeit war sehr gut und ich habe sehr viel von Lebrern und Wissenschaftlern gelernt" (S26)

- „Die Zusammenarbeit war sehr gut. Wir sind damit gut ausgekommen. Es hat mit den Lehrern und SchülerInnen gut funk.tioniert."(S32)

- „Die Zusammenarbeit war richtig gut, die Lehrer aber auch Schüler haben begeistert mitgeforscht." (S28)

- $\quad$ „Die Zusammenarbeit zwischen Lehrern und Schülern war sehr gut. Wissenschaftler haben wir nicht so oft dabei gehabt, dennoch war es eine gute Zusammenarbeit, wir konnten viel von ihnen lernen" (S24)

Einige SchülerInnen nannten konkrete Personen und Situationen, in denen die Zusammenarbeit besonders gut klappte. Hier fällt die Wissenshierarchie WissenschafterInnen(LehrerInnen)-SchülerInnen auf. 
Bei einer Antwort wurde das auch grafisch dargestellt:

Name (LehrerIn)

„WissenschaftlerIn

SchülerInnen“,

Name (LehrerIn)

wobei es laut dieser Schülerin/diesem Schüler im Verlauf des Projektes auch zu einem Lernen von- und miteinander zwischen SchülerInnen und LehrerInnen gekommen ist (S36).

Die SchülerInnen sahen sich selbst und in gewisser Weise auch die LehrerInnen als diejenigen, die primär von den WissenschafterInnen lernten. Sie fühlten sich teilweise privilegiert bzw. waren sich ihrer Sonderrolle bewusst, in dem Projekt von WissenschafterInnen lernen zu „dürfen“. Aus einer Schule kamen diesbezüglich besonders viele positive Rückmeldungen:

- „Bei diesen Projekten konnten wir von den Wissenschaftlern und den Lehrern einiges lernen. Viele praktische Dinge von unserem KV, der sebr erfahren am Berg und Gletscher ist. "(S6)

- „Die Zusammenarbeit war ricbtig gut, ich fand das auch toll, dass wir den Vortrag von Frau .... anbören durften"(S23)

- $\quad$ „Die Zusammenarbeit war gut und von dem Vortrag, den ... bielt, konnte man viel lernen“. (S34)

- „Den Vortrag von ... fand ich sehr interessant und die Zusammenarbeit mit den Lehrern hat gut funktioniert" (S25)

- „Lebrer haben uns Versuche nahe gebracht, Schüler: Versuche durchgeführt, wir lernten von Lebrern und von Gästen wie ₹:B. Schönbrunner Tiergartenpflegern,... es warperfekt, so wie es war! "(S11)

- „Wir haben viele Stunden damit verbracht alles zu lernen. Die Wissenschaftlerin hat uns viele Bilder über ibre Forschungsreisen gezeigt." (S14)

- „Was ich nicht geglaubt hätte, die Zusammenarbeit ist sebr harmonisch verlaufen. Auch als ich mit 2 Forschern auf der Pasterze unterwegs war gab es keine Probleme. "(S1)

- „Angefangen hat unser Projekt, als .... ₹u uns in die Klasse kam und uns etwas über das Projeket erzäblte, darauf hatten wir Projekttage mit den Lehrern, auch beim Ausflug ins Kübtai auf den Gossenkëllesee wurden wir von ... begleitet, dort konnten wir Eisproben ziehen und sie unter dem Mikroskop ansehen." (S2)

- „Die Zusammenarbeit war einfach nur sehr gut. Die Experten haben uns viel gelehrt und gezeigt und auch unsere Lehrer haben uns viel beigebracht. Sie waren sehr gut informiert und organisiert. Die Lehrer und auch die Experten haben uns viel anhand von praktischen Beispielen gezeigt und für mich war das eine neue und sehr interessante Art zu lernen. Natürlich war das Wetter bei diesem Projekt ein Erschwernisfaktor, da es eigentlich immer sehr kalt und nass war, dennoch hat es uns allen Spaß gemacht.."(S4)

- „Wie schon erwähnt war ich von dieser Zusammenarbeit sebr begeistert. Die Arbeit ₹wischen Schülern und Lehrern war auf jeden Fall sehr gut, da der leitende Lehrer unserer Schule auch unser KV ist. Dieser stand in guter Verbindung mit W issenschaftlern und konnte so viele Treffen und Projekte mit ibnen organisieren." 


\section{Coole Forschung}

- Das Lernen im Zuge des Kinkongs war effektiver als der Unterricht“. (S16)

Einige SchülerInnen machten Aussagen, die darauf schließen lassen, dass die SchülerInnen viel voneinander lernten und dass teilweise sogar die Wissenshierarchie WissenschafterInnen - LehrerInnen - SchülerInnen aufgeweicht wurde:

- „Wir haben alle zusammen gearbeitet, jeder hat etwas neues gelernt“ (S15)

- $\quad$ „Jeder hat von jedem gelernt, die verschiedenen Stationen Chemie, Physik, Biologie, Geografie, man konnte das lernen, was einen am meisten fasziniert" (S19)

- Alle konnten von allen lernen und es gab kaum Überordnung“(S20)

- „Schüler haben den Lehrern Fragen gestellt“ (S21)

- „Die Zusammenarbeit mit SchülerInnen und Lehrern hat bestens funktioniert. Bei den Wissenschaftern hat es genau so gut geklappt. Jeder hat etwas vom anderen gelernt." (S31)

Sechs SchülerInnen beantworteten diese Frage nicht (S9, S22, S27, S29, S30, S33).

Mehrere LehrerInnen thematisierten das Lernen untereinander. Dies fand analytisch gesehen sowohl in eher hierarchischen Settings statt, in denen „Wissende“ „Nichtwissenden" Wissen vermittelten, oder es fand ein Lernen von- und miteinander in flacheren Hierarchien statt, sodass „alle Beteiligten von allen lernen konnten“(L3). Im ersten Fall brachten die WissenschaftlerInnen als ExpertInnen den SchülerInnen mit Hilfe der LehrerInnen als Vermittlungspersonen Wissen nahe, v.a. in Vorträgen (L1, L2) und weckten so ihr Interesse und munterten sie selbst zu wissenschaftlichen Fragen auf (L2). Dabei lernten auch die LehrerInnen selbst viel (L1). Als Beispiele für Lernen in flacheren Hierarchien können Aussagen der LehrerInnen beispielsweise zum KINKONG dienen: „Alle konnten von allen profitieren. "(L2), „beim KINKONG und unserer großen Abschlussausstellung in der Schule nabmen SchülerInnen die Rolle der Lehrenden ein und präsentierten ibre Präsentationen und ibr Wissen, sind für Fragen offen,... "(L3). In der Schule einer beteiligten Lehrperson gab es eine Exkursion zum Artenschutztag in Schönbrunn, die als gemeinsames Lernen von SchülerInnen und LehrerInnen erlebt wurde.

Weitere sehr ausführliche Schilderungen über die Zusammenarbeit geben wir im Wortlaut wieder:

„Was war förderlich - genügend Zeit einplanen für Eintauchen in die Projekte, Gruppenprozesse am Gletscher, Kleingruppenarbeit und Ausarbeiten von Fragestellungen im Team,..." (L3)

„3 Projekttage und etliche Unterrichtsstunden zum Sammeln von Material, Erarbeiten der Texte, Erstellen der Homepage und des Brettspieles. 1 Projekttag im Gelände (Gossenköllesee). Fachübergreifendes Arbeiten, Vortrag von Birgit Sattler an der Schule, Film ,An Inconvenient Truth',...

Aus Sicht der SchülerInnen war vor allem die Übersetzung der Texte ins Englische eine große Herausforderung und sehr Zeit raubend. Die Erstellung einer Homepage hatten wir schon letztes Jahr geübt.

Die Klasse erarbeitete in Gruppen die erforderlichen Teilbereiche. Als Klassenvorstand und Geschichtelehrerin übernahm ich die Koordination und den Teil, der mein Fachgebiet betraf. An den Projekttagen waren auch die anderen 3 Kollegen anwesend und arbeiteten mit ibren Gruppen. Die Zusammenarbeit 
von LebrerInnen und SchülerInnen war ausgesprochen kooperativ, vielleicht auch, weil wir bereits bei anderen Gelegenheiten diese Unterrichtsform gewählt hatten.

Hindernisse für die Zusammenarbeit ergaben sich nur aus Stundenplan und Organisationsstruktur der Schule, wie etwa die Terminisierung von Projekttagen und dem Unwillen anderer KollegInnen Stunden zur Verfügung zu stellen. "(L4)

„Kontaktnahme zu den Wissenschaftlern: kein Problem - die Wissenschaftler haben sehr gern zugesagt und viel Zeit für die Schüler aufgewendet - manche haben ihr Zeitmanagement überschätzt!

Die Vorbereitungsarbeiten Wissenschaftler - Lehrer waren entscheidend über den Erfolg der Zusammenarbeit. Für mich als Lehrer muss der Workshop gut planbar sein, damit der Zeitrahmen, die Hilfsmittel und die Belastbarkeit der SchülerInnen zusammenpassen.

War die Sache gut vorbereitet, war die Zusammenarbeit SchülerInnen und Wissenschaftler sehr ertragreich. Ich glaube manche Fachexperten waren sebr überrascht, wie gezielt und ertragreich man mit SchülerInnen zusammenarbeiten kann. Die Motivation bei den Schülern war dann besonders hoch, wenn die Ergebnisse ziemlich eigenständig und eindeutig sichtbar zustande gekommen sind.

Der letzte Schritt, die Bewertung der Ergebnisse und Bedeutung der Aussagen kamen für mein Gefühl teilweise zu kurz. Hier setzen teilweise Fachleute ein kombinatorisches Denken und Verstehen voraus, das nur wenige SchülerInnen eigenständig entwickeln können.

Kritisch sehe ich die schriftliche Ausarbeitung der Ergebnisse seitens der Wissenschaftler. Wenn ich SchülerInnen mit einem GPS am Gletscher arbeiten lasse, so sollten die SchülerInnen diese Arbeit als Shapefile nachgeliefert bekommen - dann könnte man in der Klasse viele Ergebnisse besser nachvollziehen. In diesem Bereich hätte man so manche praktische Arbeit in einen größeren Zusammenhang stellen können." (L5)

Die WissenschafterInnen beschrieben die Zusammenarbeit von LehrerInnen, SchülerInnen und WissenschafterInnen folgender Maßen:

„Anfangs Brückenschlag zwischen W issenschaftern und Lehrern obne Schüler, dieser ist sehr gut gelungen (wenn die Chemie nicht stimmt, ist das beste Projekt sinnlos). Während dieser ersten Beschnupperung Austausch, was kann man machen, was wird gefordert, wie kann man es machen, wer mit wem?

Kontakt qu Schülern - Einführungsvortrag (mit Lehrern)

Exkursion ins Hochgebirge auf Forschungsstation etc.

Die Lehrer zeigten den Wissenschaftern, wo sich die Schüler geistig befinden (wichtige Hilfe zur Niveaufindung des Wissenschafters), versuchten immer, das Gebotene der Forschungseinrichtung in den Lehrplan zu integrieren.

Die Schüler zeigen dem Wissenschafter oft Fragen auf, mit denen er/ sie sich noch nie beschäftigt hat (z.B. schlafen Bakterien?).

Förderlich war die außergewöhnliche Flexibilität und Bereitschaft der Lebrpersonen der Schulen in Tirol, Ideen, welche oft auch spontan ausgedrückt wurden, mit den Kindern zu bearbeiten. Förderlich ist auch die Nähe zu den Schulen. 


\section{Coole Forschung}

Förderlich im Fall Tirol war die Zusammenstellung des Teams, welches auch viel dafür in der Freizeit gearbeitet hat - mit diesen Leuten ist die Arbeit sehr erleichtert worden. Danke auch der Projektleitung in Wien!!!! Dr. Wolfgang Schöner hat das Projekt äußerst professionell und kollegial geleitet.

Hinderlich sind oft große zeitliche Lücken zwischen den Besuchen bzw. Veranstaltungen, es wäre effizienter, wenn es derartige Projekttage gäbe, in welchen man am Thema bleiben kann. Andererseits kann man diese Kooperation über das gesamte Jahr hinziehen und die Schüler wachsen damit (Beispiel HS Zirl, die wir von der 1. Klasse begleiten durften, nun in der 3.) "(W1)

„Kontaktaufnahme W(issenschafter) - L(ehrerInnen), 1. Workshop, Besprechung der Teilnahme, Aktivitäten. Austausch von Unterrichtsmaterialien per Mail (L an L, W and L). Exkursionen geplant - enger Kontakt zw. jeweiligen W und L.

Dann S(chülerInnen) eingebunden. Vorbereitung auf das Thema im Unterricht durch L. W kamen auf Wunsch zu Kurzreferaten an die Schulen. Einige Klassen besuchten Uni und ZAMG, konnten selber Versuche durchführen, mikroskopieren etc.

W, L und S gemeinsam auf Exkursionen z: B: auf die Pasterze: gemeinsame Datenerhebung, Gletschermessung. Auch für L neu und spannend. Engagierte und motivierte S! W lernten Zeitmanagement: Kinder und Jugendliche geben anderes Tempo vor. Außerdem erbielten $W$ Einblick in die für solche Aktivitäten schwierigen Rahmenbedingungen in der Schule.

Entwicklung eigener Forschungsfragen gestaltete sich schwierig - Anregungen von Seiten der W waren immer nötig! S und L können ja nicht wissen, was Stand der Wissenschaft ist, was machbar ist etc. ...

Kinderkongress an der Uni: über 200 Kinder und Jugendlich aus West- und Ostösterreich: ausgezeichnete Präsentationen und Versuchsstände. S lernten das "Verkaufen" erhobener Daten.

Positiv: Gesprächsbasis zw. $L$ und $W$ auch zw. $W$ und $S$ war von Anfang an gegeben. Ziel einer Lernebene wurde meiner Meinung nach erreicht.

Hinderlich ist sicher das nicht so flexible System Schule - Exkursionen müssen länger geplant werden und können nicht spontan, wenn ₹.B. das Wetter nicht passt, verschoben werden. "(W2)

„So wie im Projektantrag vorgesehen (bier nur die ZAMG Aktivitäten):

1) Brückenschlag WissenschaftlerInnnen LehrerInnen beim Eingangsworkshop in Wien (der geplante, im Gebirge konnte auf Grund des Wetters nicht stattfinden). Von Seite der Schulen wurden Vorgaben von den WissenschaftlerInnen erwartet. Das wurde versucht auf ein Minimum zu beschränken. Die Vernetzung hat gut funktioniert und der Teamcharakter hat sich sehr gut entwickelt.

2) Wissenschaft in die Schule: Für die ZAMG waren das im Wesentlichen die Besuche der Schulen an $\operatorname{der} Z A M G$ bzw. die Besuche von ZAMG-WissenschaftlerInnen in den Schulen. Damit auch der erste Kontakt zu den Schülern. Das Umschalten der Wissensvermittlung (von Gegenüber „Wissenschaftler") auf Gegenüber „Schüler" hat etwas Ansprüche an die WissenschaftlerInnen gestellt. Die Rollen waren noch sehr klassisch „,der Wissenschaftler erzählt den Schülern“. Das wurde insbesondere von der Lehrern auch stark eingefordert. Der/ die WissenschaftlerIn schlüpfte somit kurzzeitig die Rolle des Lehrers/Lehrerin. Gut angekommen sind dabei die Besuche an der 
ZAMG mit Wetterbütte, Wetterballonaufstieg, ....

Der nächste Schritt und bereits Übergang zu 3) waren die gemeinsamen Unternehmungen im Gebirge, bei denen die ScbülerInnen einerseits direkt bei den Arbeiten der WissenschaftlerInnen dabei sein konnten und andererseits kleinere selbst überlegte wissenschaftliche Fragestellungen durchfübren konnten (Probennabmen, Messungen). Hier konnten die Scbüler, basierend auf ibrem erworbenen Wissen, bereits selbst aktiv den Projektverlauf mitbestimmen und mit den WissenschafterInnen als PartnerInnen kommunizieren.

3) Wissenschaftler der Zukunft: Im Zentrum stand der KingKong bei dem die durchgeführten Arbeiten aufbereitet und präsentiert wurden. Die Veranstaltung wurde an der Uni Wien durchgeführt um den wissenschaftlichen Flair zu verstärken. Die Motivation aller Beteiligten war enorm. Die WissenschaftlerInnen und LehrerInnen waren in der Rolle der Organisatoren die SchülerInnen in der zentralen Rolle der Akteure. Neben Vorträgen waren auch Experimente, Zeichnungen, Videos etc. Gegenstand des Kongresses, der sehr viel Raum für Diskussionen zwischen allen Beteiligten und auch den Auftraggebern (BMWF) bot.

4) Letater Teil des Projektes warIst das "Gehen nach draußen", also die Publikationen und die Gestaltung von Ausstellungen. Die Gestaltung wird vorwiegend von WissenschaftlerInnen und LehrerInnen bestimmt. Die ScbülereInnen bringen sich vorwiegend über Inhalte ein. Ausstellungen werden von mebreren Schulen durchgefübrt, teilweise auch gemeinsam. "(W3)

„Neben inhaltlicher Arbeit erwarben die ScbülerInnen laut einer Person des Wissenschaftlichen Teams folgende Fähigkeiten: „Erlernen von Präsentieren von wissenschaftlichen Inhalten (KinKong, Sparkling Science Schulpreis) mit/ obne IT (je nach Alter), Kommunizieren: Kontaktaufnabme zu internationalen Wissenschaftern auf Englisch"(W1).

\section{Aspekte von BiPolar, die stärker beachtet oder verändert werden sollten}

Eine Frage, die auf das Erfassen von Entwicklungspotentialen des Projekts gerichtet war, wurde von den Probanden und Probandinnen unterschiedlich interpretiert.

Einige SchülerInnen verstanden die Frage nach Verbesserungspotentialen im Sinn von: „Sollte so ein Projekt wieder durchgeführt werden?“ Die Antworten auf diese Frage sind selbstverständlich interessant und wir führen sie hier gemeinsam mit den Antworten auf die Frage, wie wir sie intendiert hatten, an. Sie reichten von kurzen "Ja", teilweise mit Rufzeichen (S1, S11, S14, S25) über ausführlicher formulierte positive Aussagen wie: „Ja sollte es! Es muss nichts stärker beachtet werden." (S8), "Ja, ich finde wir sollten äbnliche Projekte durchfübren" (S32), eine Aussage, die in ganz ähnlicher Formulierung zwei andere SchülerInnen ebenfalls machten (S33, S34). „Ich finde es war gut so wie es ist und JA es wäre eine sebr gute Idee so etwas noch einmal zu machen “(S4), „Ja, natürlich, wenn es einem guten Zweck dient"(S10), „Nichts! Ich finde das Projekt war super!". "Ja es sollte so ein äbnliches Projekt wieder durchgeführt werden, damit den Kindern näher gebracht wird, wie es um unsere Welt eigentlich ausschaut. "(S28)

Etwas weniger enthusiastische Formulierungen gab es auch: „wäre schon ok"(S15), „Es war gut genug, deswegen weiß ich nicht was man verbessern sollte" (S27), "Ja, meinetwegen. [wohl bezogen auf den zweiten Teil der Frage, Anm. Evaluationsteam] Keine Abnung. "(S19) 


\section{Coole Forschung}

Drei SchülerInnen sind gegen eine nochmalige Durchführung eines ähnlichen Projekts: „Nein, da man viel zu viel theoretisches unternommen hat" (S12), „Nein, persönlich hat es mir nicht gefallen“(S17), „Nein, lieber nicht"(S21).

Konkrete Änderungswünsche wurden von einigen SchülerInnen vorgebracht. Sie betreffen ganz unterschiedliche Aspekte des Projekts und werden daher großteils wörtlich wiedergegeben:

Drei SchülerInnen wünschten sich mehr Lehrausgänge, Ausflüge oder Exkursionen (S2, S22, S31), z.B.: „Ich finde wir sollten mehr Ausflüge, passend zum Projek.t unternehmen, da die Schüler sich dadurch mehr für die Projekte interessieren würden" (S31)

Den Wunsch nach mehr Vorträgen artikulierten zwei SchülerInnen (S22, S26), den nach mehr Versuchen ebenfalls zwei (S26, S30) und den nach einer kürzeren Projektdauer ebenfalls zwei (S7, S36).

Andere Änderungswünsche betrafen didaktische Aspekte:

- „man sollte mehr Geräte für verschiedene Messungen zur Verfügung stellen. “(S5)

- mehr Informationszettel“" (S26)

- „mehr Filme“ (S35)

- „mehr Sachen wie KinKong“(S16)

- „ „es sollte mehrere Fächer übergreifen“(S24)

- „Der Kongress am Schluss sollte interessanter sein sonst war alles ok.“"(S3).

Eine weitere Anzahl von Aussagen bezog sich u.a. auf soziale Aspekte im Projekt:

- man sollte noch mehr Wissenschaftler einsetzen“(S5)

- Es sollte öfters Treffen mit allen Beteiligten geben. (wie King Kong)“(S6)

- Die Vermittlung mit Menschen außerhalb vom Projekt" (S9)

- Es sollten mehrere Schulen teilnehmen, brw. auch Schulen aus dem Ausland“(S13)

- „es sollte mehr auf die Verbindung mit der Jugend geachtet werden“ (S20)

- „Ja, das wäre ganz ok wenn das wieder durchgeführt werden würde aber wenn wirklich alle so richtig mitmachen und es wirklich wollen. Wenn aber nicht dann lieber nicht weil es soll allen Spaß machen und nicht nur einigen." (S29)

Zeit wurde von mehreren LehrerInnen als limitierender Faktor identifiziert. Einerseits könnte das Projekt kürzer dauern, um die Problematik zu verhindern, dass SchülerInnen nach der 4. oder 8. Klasse AHS aus dem Projekt ausscheiden (L2). Andererseits ging es um die langen Planungshorizonte in Schulen: „Zeitressourcen der Schulen erfordern exakte und rechtzeitige Planung. Informationen sollten daher möglichst früh und genau erfolgen. “(L4) „Die Planungszeiträume waren teilweise zu kurz; in der Schule sind die Abläufe sehr träge, weil großteils sehr langfristig geplant werden muss (Schularbeiten, andere Verpflichtungen der Schüler). "(L5) 
Bei einem zukünftigen Projekt könnten außerdem folgende Aspekte stärker beachtet oder verändert werden:

- „Die Zusammenarbeit unter den Schulen“(L1)

- $\quad$ „Ein Workshop mit Wissenschaftlern als Vortragende/Gestaltende und den SchülerInnen als TeilnehmerInnen wäre toll. "(L4)

- „Die finanziellen Möglichkeiten sollten von Anfang an klar definiert sein, da derartige Projekte relativ hohe Anforderungen an die Finanzkraft der Eltern stellen." (L5)

Eine Lehrperson meinte, dass ,nichts “stärker beachtet oder verändert werden sollte (L6).

Die WissenschafterInnen machten folgende Aussagen zu dieser Frage:

„Diese Programme gehören meiner Meinung nach etabliert, d.h. im Idealfall sollte es gelingen, im Curriculum einer Schule eine Kooperation mit einer Forschungseinrichtung (Uni, FH, o. ̈..) zu verankern.

$W$ as bei einigen Schulen ein Stolperstein für zusätzliche Ideen war, war eine teilweise Unflexibilität der Schulen, die jedoch nichts mit der Bereitschaft des Lebrpersonen zu tun hatte, sondern mit dem Korsett des Lebrplanes. Es hängt sehr stark vom Schultyp ab, wie frei die Schule für zusätaliche Veranstaltungen, Exkursionen, Labortage, Programmänderungen außerhalb des Lehrplanes ist. Ein äußerst positives Beispiel ist die Ökolog-Hauptschule Zirl, bei anderen Schulen jedoch war der Lehrplan zu straff, was die Durchführung derartiger Veranstaltungen äußerst schwierig machte.

Wenn diese Programme vom Ministerium weiterhin gewünscht werden, so müsste man dies auch im Bildungsministerium dementsprechend deponieren, um den Lehrern innerhalb ibrer Dienstzeit die erforderliche Flexibilität zu geben.

Bei einer wiederholten Einreichung bei Sparkling Science müsste auch unbedingt darauf geachtet werden, dass etwaige Fahrten der gesamten Klasse aus diesem Budget finanziert werden können. Im konkreten Beispiel der HS Zirl konnten die Gletschertage nur über die gewonnenen Preisgelder durchgeführt werden. Obne diese zusätzlichen Gelder bleibt die Forschungsbildungskooperation im Klassenzimmer (und nur dort ist fad). "(W1)

„Längerfristige Planung, sodass L die Möglichkeit haben, das Projekt ins nächste Schuljahr einzuplanen (limitierte Projekttaganzahl!) "(W2)

„Stärkere Vernetzung von Wissenschafts- und Unterrichtsministerium und den untergeordneten Stellen: Landesschulinspek.toren kannten (vielleicht jet:t schon) Sparkling Science oft gar nicht!" (W2)

„Frühere Einbindung der Schulen in die Projektplanung. Bessere finanzielle Abdeckung der Kosten für Schulen (insbesondere für die ScbülerInnen) "(W3)

Bereitschaft der Akteure zur Mitarbeit in einem ähnlichen Projekt

33 SchülerInnen würden bei einem ähnlichen Projekt wieder mitmachen, drei nicht. Bezüglich der Gründe fürs weitere Mitmachen herrschte große Einigkeit unter den SchülerInnen: 


\section{Coole Forschung}

Das Projekt war „interessant"(14 Nennungen), es gefiel ihnen gut und machte ihnen großen Spaß (9 Nennungen), es war lehrreich (8 Nennungen). Zwei SchülerInnen erwähnten, dass das Projekt die Klassengemeinschaft und Zusammenarbeit unter den SchülerInnen gefördert hätte (S6, S36), zwei andere, dass es ,einen guten Zweck“ (S33) gehabt hätte bzw. „,hilfreich"war (S10).

Andere SchülerInnen erwähnten folgende weitere positive Aspekte. Bemerkenswert aber keineswegs überraschend ist dabei, dass sie als Bequgssystem den ,normalen“ Schulalltag verwenden:

- „ , besonders die zablreichen Exkursionen“. (S5)

- „weil es eine gute Abwechslung zum öden Schulalltag ist“. (S6)

- weil ich eine bessere Note baben will“(S21)

- „weil wir nur in Bio Projekte machen“ (S30)

- „weil es wenig anstrengend ist“ (S35).

Drei SchülerInnen würden bei einem ähnlichen Projekt nicht mitmachen wollen,

- „weil es mir nichts bringt außer einem schlechten Gewissen“(S9)

- „weil die praktische Seite vernachlässigt wurde“(S12)

- „Persönlich hat es mir nicht gefallen" (S17).

Alle LehrerInnen zeigten hohe Bereitschaft, an einem ähnlichen Projekt wieder teilzunehmen. Die Begründungen waren vielfältig und thematisierten die besonderen Lernmöglichkeiten für die SchülerInnen, aber auch für die LehrerInnen selbst:

- „weil es Neues bringt - auch für mich interessanter ist, bei den Schülern sicher mehr ankommt... " (L1)

- $\quad$ „weil wir durch den Kontakt mit den WissenschaftlerInnen unseren fachlichen Horizont erweitert haben und für SchülerInnen der unmittelbare Kontak.t mit den WissenschaftlerInnen wertvoll ist" (L2)

- ـweil die Inhalte für die SchülerInnen auf diese Weise viel konkereter und anschaulicher sind und die Erfabrungen so nachbaltiger bleiben. "(L3)

- $\quad$ „weil das gemeinsame Arbeiten an einem großen Projekt eine andere Form des Lernens und Zusammenarbeitens zwischen Lehrern und SchülerInnen ermöglicht. "(L4)

- , weil Projekte die Unterrichtsarbeit beleben und die betroffenen Fächer davon stark profitieren." (L5)

- $\quad$ „weil unser schulisches Umfeld solchen Projekten eher positiv gegenübersteht und die schulischen Rahmenbedingungen sebr flexibel sind. "(L5)

- „weil ich persönlich meinen Arbeits- und Wissenshorizont erweitern kann“ (L5) 
- $\quad$ „weil solche Projekte großteils ein ganzheitliches Lernen bewirken, etwa Übernachten mit Gleichgesinnten, Organisation einer Selbstversorgerbütte, Erleben von schwierigen, nicht vorhersebbaren Situationen und die eher partnerschaftliche Begegnung Lebrer - SchülerIn. "(L5)

- „weil ich persönlich, aber auch die Schüler in vielfacher Hinsicht profitiert haben. "(L6)

Eine einzige Lehrperson gab jedoch zu bedenken, dass , die Anforderungen von Sparkling Science mit Berichten etc. sehr zeitintensiv sind."(L3)

Die drei WissenschafterInnen beantworteten die Frage alle positiv:

„Ja, weil...Unbedingt, ich sehe die Zusammenarbeit zwischen Wissenschaft und Schule als eine wichtige Einrichtung, da Schüler dabei eine neue Sichtweise der Fragestellung bekommen, lernen, wie Experimente geplant werden sollten, lernen viel Teamgeist. Für den Wissenschafter ist es eine herzerfrischende Herausforderung, Wissenschaft so zu gestalten, dass es Spaß macht und verständlich ist, zudem für die Kinder (be)greifbar ist. Zudem sehe ich es als Verpflichtung, brisante Forschungsfragen auch mit den Wissenschaftern der Zukunft zu diskutieren bzw. sie ibnen mitzugeben. "(W1)

„Ja, weil ... die Zusammenarbeit von $L, S$ und $W$ jedem etwas bringt, die Wissenschaft wird entmystifiziert, engagierte L haben die Möglichkeit dem Alltag Schule teilweise zu entfliehen und S können praxisnäher lernen. "(W2)

„Ja, weil das Team hochmotiviert war und die Zusammenarbeit mit allen Teilnehmern Spaß gemacht bat." (W3)

\section{Weitere Blitzlichter auf die Kooperation}

Sieben SchülerInnen machten Anmerkungen. In diesen äußerten sie ihre Hoffnung auf weitere Projekte (S13, S23, S32), wiederholten ihnen wichtige Aspekte, z.B. S 36: „,kürzer, Gletscher war sehr lehrreich, Ich fand den Kinkong sehr gut." oder äußerten Gedanken, für die sie im Fragebogen bis dahin keinen Platz fanden:

- „Man sollte belfen wo man kann“(S10)

- „Umsetzung ganz gut, Routine feblt“ (S16)

- „Ich hätte mir sehr gern erhofft, dass wir auch auf einen Berg erforschen gehen. Leider hat es nicht geklappt."(S31)

Zwei LehrerInnen machten folgende Aussagen:

- Großer Zeitdruck war eine Belastung. “(L4)

- „Es war eine tolle Chance über ein Thema zu arbeiten, über das wir vorber nicht so viel wussten und das sehr aktuell ist!" (L4)

- „Ich denke, ein Großteil der Mitarbeiter kann sich ein Folgeprojekt mit neuen Zielsetzungen gut vorstellen."(L5) 
Bei den weiteren Anmerkungen schrieben zwei WissenschafterInnen Folgendes:

- „Kann nur allen Beteiligten danken für diese großartige Erfahrung! Was mir feblt, ist eine weitgehende Akzeptanz für derartige Projektmodelle an der Universität selbst."(W2)

- „Mö̈hte dem gesamten Team (LebrerInnen, SchülerInnen, WissenschaftlerInnen) für die gute Zusammenarbeit und tolle Zeit danken." (W3)

\subsubsection{Schlussfolgerungen}

- Diejenigen Beteiligten, die eine Rückmeldung machten, sind insgesamt sehr zufrieden mit dem Projekt. Sie führten eine Vielzahl unterschiedlicher positiver Aspekte an, abhängig von der jeweiligen Perspektive als SchülerIn, LehrerIn oder WissenschafterIn.

- Sehr viel von dem, was sich die Beteiligten selbst erwartet haben, hat sehr gut geklappt. Ansonsten wäre viel mehr Kritik bei der Frage nach weniger gelungenen Aspekten von BiPolar vorgebracht worden und es wären viel mehr und/oder viel weiterreichende Vorschläge für Veränderungen oder Verbesserungen bei einer nochmaligen Durchführung aufgelistet worden.

- Die Zusammenarbeit zwischen den Akteuren klappte bis auf einige Details ausgezeichnet.

- Die SchülerInnen und LehrerInnen empfanden die Mitarbeit in praktischen Forschungssettings, die Teilnahme an Exkursionen und die Durchführung von Versuchen als besonders motivierend. Dadurch lernten sie viel über die Arbeit der WissenschafterInnen.

- Die Partizipation der SchülerInnen schien in unterschiedlichen Schulklassen und Schulen unterschiedlich ausgeprägt. Sie war für die meisten SchülerInnen besonders beim Kinderkongress möglich.

- Es gab einen starken Fokus auf sachbezogenes, inhaltliches Arbeiten. Dies wurde einerseits durch die teilnehmende Beobachtung in Modul 1, andererseits durch viele Aussagen in den Fragebögen ersichtlich. Die Kommunikation und Arbeitsbasis zwischen den Gruppen wurde durch dieses sachbezogene Arbeiten gelegt. Es wurde vergleichsweise weniger Aufmerksamkeit auf die Konstitution von Gruppen- und Kommunikationsprozessen gelegt. Dadurch wurde intensiver Kommunikation zwischen den einzelnen Schulen weniger gefördert, was manche der LehrerInnen in den Fragebögen im Nachhinein als wünschenswert erachtet hätten. Auch das Wissen um sozialwissenschaftliche Aspekte von Forschung (Was machen ForscherInnen in ihrem Alltag? Wie kommunizieren sie untereinander und mit anderen? etc.) schien bei LehrerInnen und SchülerInnen großteils implizit zu bleiben.

- Wie in vielen Forschungs-Bildungskooperationsprojekten zeigt sich teilweise eine Hierarchie des Wissens. WissenschafterInnen vermitteln Wissen an LehrerInnen und diese vermitteln (tw. gemeinsam mit den WissenschafterInnen) Wissen an die Schüle- 
rInnen. Es gibt wenige Hinweise darauf, dass die SchülerInnen die Fragen der WissenschafterInnen beeinflusst haben.

- Das Design des Projekts ist hochkomplex, u.a. durch die große Zahl der Beteiligten! Die Rahmenbedingungen in den Schulen sind unterschiedlich, stellen aber u.a. auf Grund der langen Planungshorizonte insgesamt ein viel engeres Korsett dar als die Universitätsstrukturen. Forschungs-Bildungs-Kooperationen sind selten, daher müssen Projekte wie BiPolar gerade in den Schulen und übergeordneten administrativen Instanzen (Stadtschulrat, Bezirksschulrat, Landesschulrat) Pionierarbeit leisten, was für die LehrerInnen auch belastend sein kann.

Ein übergeordnetes Ziele von BiPolar war der Brückenschlag zwischen Forschung und Schule: Bridging Science and School. Vieles spricht dafür, dass dieses Ziel erreicht wurde. Die Daten zeigen eine ungemein positive Einstellung der Schülerinnen und Schüler zu naturwissenschaftlicher Forschung. Sie wurden demnach nicht enttäuscht. Wissenschaftlerinnen und Wissenschaftler stellen durchaus mit dem Unterton der Überraschung fest, dass die eigenen Fragen sich durch die Diskussion mit den Partnern positiv entwickelt haben. Die berichteten Erfahrungen der Teilnehmerinnen und Teilnehmer legen nahe, die Ursachen für diesen erfolgreichen Brückenschlag an Personen, ihrer Kompetenz und Kooperationsbereitschaft festzumachen.

Eine wahrscheinlich bedeutendere Ursache für das Gelingen wird kaum in den Rückmeldungen in den berichteten Projektaktionen aber klar sichtbar. Neben der Attraktivität des Themas wurde vom BiPolar Team auch ein anderes wesentliches Element erfolgreicher Forschungs-Bildungs-Kooperation umgesetzt: Polarforscherinnen und Polarforscher haben für die Kooperationspartner und vor allem für die Schülerinnen und Schüler ihre Forschungshaltungen- und die Details des Forschungsprozesses offen gelegt. Sie gewährten den Partnern Einblicke in die spezifischen, für Laien normalerweise nicht zugängigen „Geheimnisse“ ihrer Wissenschaften: Backstage of Science. Der gelungene Brückenschlag in BiPolar erscheint daher aus der Perspektive der Evaluation auch als gelungene Enculturation in communties of experts. 


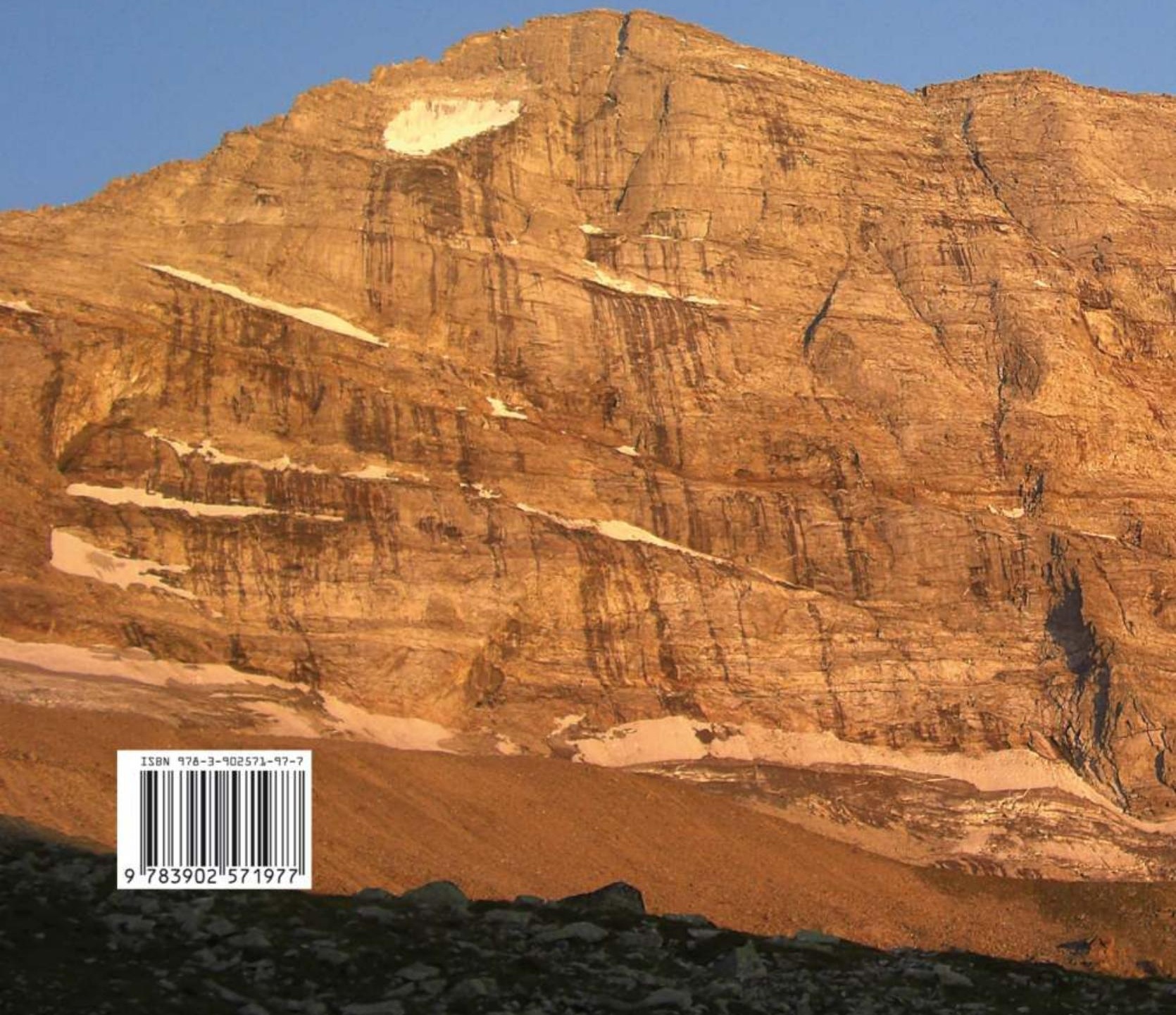

